\title{
Strategies to improve breast cancer detection by digital screening mammography
}

Citation for published version (APA):

Coolen, A. (2022). Strategies to improve breast cancer detection by digital screening mammography. [Doctoral Thesis, Maastricht University]. Ipskamp Printing BV. https://doi.org/10.26481/dis.20220127ac

Document status and date:

Published: 01/01/2022

DOI:

10.26481/dis.20220127ac

Document Version:

Publisher's PDF, also known as Version of record

\section{Please check the document version of this publication:}

- A submitted manuscript is the version of the article upon submission and before peer-review. There can be important differences between the submitted version and the official published version of record. People interested in the research are advised to contact the author for the final version of the publication, or visit the DOI to the publisher's website.

- The final author version and the galley proof are versions of the publication after peer review.

- The final published version features the final layout of the paper including the volume, issue and page numbers.

Link to publication

\footnotetext{
General rights rights.

- You may freely distribute the URL identifying the publication in the public portal. please follow below link for the End User Agreement:

www.umlib.nl/taverne-license

Take down policy

If you believe that this document breaches copyright please contact us at:

repository@maastrichtuniversity.nl

providing details and we will investigate your claim.
}

Copyright and moral rights for the publications made accessible in the public portal are retained by the authors and/or other copyright owners and it is a condition of accessing publications that users recognise and abide by the legal requirements associated with these

- Users may download and print one copy of any publication from the public portal for the purpose of private study or research.

- You may not further distribute the material or use it for any profit-making activity or commercial gain

If the publication is distributed under the terms of Article $25 \mathrm{fa}$ of the Dutch Copyright Act, indicated by the "Taverne" license above, 
Strategies to improve breast cancer detection by digital screening mammography 
(C) Angela Coolen, Maastricht 2022, All rights reserved

\author{
Cover design: Wendy Bour \\ Layout: Tiny Wouters Lenssen \\ Production: Ipskamp Printing
}

ISBN: 978-94-6421-618-9 


\title{
Strategies to improve breast cancer detection by digital screening mammography
}

\author{
PROEFSCHRIFT \\ Ter verkrijging van de graad van doctor aan de Universiteit Maastricht, \\ op gezag van de Rector Magnificus, Prof. dr. Rianne M. Letschert, \\ volgens het besluit van het College van Decanen, \\ in het openbaar te verdedigen \\ op donderdag 27 januari 2022 om 10.00 uur \\ door \\ Angela Maria Petrus Coolen \\ Geboren op 7 april 1988 te Eindhoven
}




\section{Promotor}

Prof. dr. V.C.G. Tjan-Heijnen

\section{Co-promotores}

Dr. L.E.M. Duijm, Canisius Wilhelmina Ziekenhuis, Nijmegen

Dr. A.C. Voogd

\section{Beoordelingscommissie}

Prof. dr. M.L. Smidt (voorzitter)

Prof. dr. L.J. Boersma

Prof. dr. M.J.M. Broeders, Radboud Universiteit, Nijmegen

Prof. dr. J.W.M. Muris

Prof. dr. R.M. Pijnappel, UMC, Utrecht 


\section{Contents}

$\begin{array}{lll}\text { Chapter } 1 & \text { General introduction } & 7\end{array}$

Chapter $2 \quad$ Impact of the second reader on screening outcome at 15

blinded double reading

(British Journal of Cancer, 2018)

Chapter $3 \quad$ Characteristics of screen-detected cancers following 29 concordant or discordant recalls at blinded double reading (European Radiology, 2018)

Chapter $4 \quad$ Value of technologists' reading and training on outcome at screening mammography

4.1 Incorporation of the technologist's opinion for arbitration of discrepant assessments among radiologists at screening mammography (Breast Cancer Research and Treatment, 2018)

4.2 Additional breast cancer detection at digital screening mammography through quality assurance sessions between technologists and radiologists (Radiology, 2020)

Chapter $5 \quad$ Frequency and characteristics of additional breast abnormalities following recall at screening mammography

5.1 Frequency and characteristics of additionally detected contralateral breast abnormalities (European Radiology, 2018)

5.2 Frequency and characteristics of additionally detected ipsilateral abnormalities

(The Breast, 2018)

Chapter $6 \quad$ General discussion and future perspectives

Summary

Impact paragraph

Dankwoord

Curriculum vitae 

Chapter 1

General introduction 



\section{General introduction}

With over 2.2 million new cases in 2020, breast cancer is the most common cancer diagnosed in women worldwide. ${ }^{1}$ In the Netherlands, one in seven women is diagnosed with the disease during her lifetime. ${ }^{2}$ Breast cancer screening programmes aim at reducing breast cancer mortality through detection and treatment at an early stage. However, there is continuing controversy about the effectiveness and efficacy of breast cancer screening. In 2014, in a report of the Health Council of the Netherlands, it was concluded that the Dutch nationwide breast cancer screening programme is as effective as was originally expected, even though circumstances have changed since its introduction in $1989 .{ }^{3}$ Since then, breast cancer mortality has declined by 30-34\%. ${ }^{4}$ Reported breast cancer mortality before 1997 was 91.6/100,000 in Dutch women aged between 50 and 74 years, ${ }^{5}$ which was reduced to $51.3 / 100,000$ in 2018. ${ }^{6}$ This is the lowest reported mortality rate since the introduction of the breast cancer screening programme. This decrease in mortality throughout the years has been attributed to the introduction of breast cancer screening (resulting in earlier detection), substantial improvements in treatment and increased breast cancer awareness among women.

\section{Dutch breast cancer screening programme}

After two pilot projects in Utrecht and Nijmegen, ${ }^{7,8}$ the Dutch nationwide breast cancer screening programme was implemented gradually from 1989, reaching its full population capacity in 1997 . The programme originally offered biennial screening mammography to asymptomatic women aged between 50 and 70 years. In 1998, the upper age limit was extended to 75 years. The Breast Imaging Reporting and Data System (BI-RADS) was implemented in our screening programme in 2008 and has been routinely used and mentioned in recall reports since $2009 .{ }^{9}$ Women with a BI-RADS 0,4 or 5 abnormality are routinely recalled and referred by their general practitioner to a dedicated breast unit of a nearby hospital for further evaluation.

\section{Reading strategies}

Different reading strategies may be used in the assessment of screening mammograms. These vary from single reading, with or without computer-aided detection, to double reading, which may be performed in either a non-blinded (independent) or blinded fashion. In the latter case, the second reader is blinded to the first reader's decision. Previous studies have shown that non-blinded double reading significantly increases the cancer detection rate (CDR) compared to single reading. ${ }^{10,11}$ Another study compared blinded reading with non-blinded double reading and found a higher CDR and programme sensitivity at blinded double reading, 
at the expense of an increased recall rate and false positive recall rate. ${ }^{12}$ Most of these studies date from the screen-film mammography (SFM) era, this thesis prospectively evaluates the value of radiologist blinded double reading compared to single reading at digital mammography screening.

In accordance with European guidelines, ${ }^{13}$ double reading is already standard of care in our screening programme. Double reading, either non-blinded or blinded, generates discordant readings between two readers. A discordant reading is defined as a difference in classification between two readers where only one of the readers considers recall necessary. There are different strategies on how to deal with these discordant readings. For example, one might choose to recall all women with a discordant reading, try to reach consensus between both readers or add arbitration by a third reader or panel of readers. Several studies have explored these consensus and arbitration strategies and their effect on screening outcome. ${ }^{14-19}$ In this thesis we explore which mammographic and tumour characteristics lead to concordant versus discordant recalls at blinded double reading.

\section{Technologists' training and quality assurance}

In the Dutch screening programme, mammograms are obtained by specialised mammography technologists. They receive extensive training before being employed in the nationwide screening programme. Although mammography technique and positioning are central to their training, technologists are also trained in breast anatomy and mammographic characteristics of benign and malignant breast diseases. During their employment as screening technologists, they receive further training yearly and are required to attend mammography symposia and congresses on a 1-2 yearly basis. Since the initiation of our screening programme, technologists have been encouraged to look for mammographic abnormalities after obtaining the mammogram. Previous studies have investigated the role of technologists as screening mammography readers. ${ }^{10,20-24}$ Possible reading strategies using technologists as readers include double reading by a radiologist and technologist or using the technologist's reading to arbitrate discordant readings among radiologists. The first could especially be of value in screening programmes with a shortage of screening radiologists, as an alternative to radiologist double reading. In our nationwide screening programme, there is no such shortage and technologists do not function as official readers. However, in our current screening setting, they do read each mammogram directly after obtaining it. The technologist annotates whether she would recall the woman in question and classifies the mammogram according to BIRADS. One of the objectives of this thesis is to determine whether this technologist's reading might be helpful in deciding which discordant readings at radiologists blinded double reading should be recalled. 
As part of their continued training as well as for the purpose of quality assurance, technologists attend 6-weekly quality assurance sessions. Under supervision of a coordinating screening radiologist, they discuss interval cancers and other instructive cases. They also discuss all mammograms with abnormalities that were considered suspicious by the technologists but did not result in recall at radiologist double reading. Additional women are recalled through these sessions if the coordinating radiologist considers recall necessary after all, and we know that this results in additional screen-detected cancers. Insight into frequencies as well as mammographic and tumour characteristics of these additionally detected cancers could provide us with a better understanding of what makes radiologists miss these cancers. This is therefore a focus of this thesis.

\section{Additionally detected abnormalities}

Women are usually recalled for a solitary, unilateral abnormality. The percentage of bilateral recalls is only $1-2 \%$ and a previous study performed in our screening region showed that the sensitivity of mammography screening for bilateral breast cancer detection is low. ${ }^{25}$ Over the years, there have been substantial improvements in breast imaging techniques (for example transition from SFM to full-field digital mammography (FFDM) and, more recently, digital breast tomosynthesis (DBT) and MRI) for the detection of breast abnormalities. In clinical work-up after recall, additional breast imaging is routinely performed to further characterise a recalled abnormality. This may result in additionally detected abnormalities in the ipsilateral or contralateral breast. In some cases, these lesions are found through pre-operative MRI. ${ }^{26,27}$ Additionally detected, non-recalled, lesions prove to be benign in most cases. However, they also comprise malignancies, either satellite lesions associated with a recalled malignancy or a synchronous breast cancer. Data on frequency and characteristics of these additional ipsilateral and contralateral abnormalities is lacking. We know that a false positive recall generates stress and might even effect reattendance rates. It is therefore also important to study if and to what extend these additional lesions add to the false-positives and what kind of work-up they require. These are objectives of this thesis.

\section{Objectives}

1. To determine the impact of the second reader on screening outcome at blinded double reading.

2. To analyse which mammographic and tumour characteristics lead to concordant versus discordant recalls at blinded double reading.

3. To determine the value of technologists' reading and training on screening outcome. 
4. To determine the frequency and characteristics of contralateral and ipsilateral non-recalled breast abnormalities following recall. 


\section{References}

1. World Health Organization. Global Cancer Observatory: Cancer Today. Available from: https://gco.iarc.fr/today/online-analysis-table?v=2020\&mode=cancer\&mode_population= continents \&population $=900 \&$ populations $=900 \& k e y=a s r \& s e x=2 \&$ cancer $=39 \&$ type $=0 \&$ statistic $=5 \&$ pre valence=0\&population_group=0\&ages_group $\% 5 \mathrm{~B} \% 5 \mathrm{D}=0$ \&ages_group $\% 5 \mathrm{~B} \% 5 \mathrm{D}=17$ \&group_cancer $=1$ \&include_nmsc=1\&include_nmsc_other=1 [Accessed 5th May 2021].

2. Netherlands Comprehensive Cancer Organisation. Borstkanker in Nederland. 2018.

3. Health Council of the Netherlands. Population screening for breast cancer: expectations and developments. 2014.

4. Sankatsing VDV, van Ravesteyn NT, Heijnsdijk EAM, Looman CWN, van Luijt PA, Fracheboud J, et al. The effect of population-based mammography screening in Dutch municipalities on breast cancer mortality: 20 years of follow-up. Int J Cancer. 2017;141(4):671-677.

5. Erasmus MC, Radboud UMC, Bevolkingsonderzoek borstkanker: Monitor 2014. 2016.

6. Netherlands Comprehensive Cancer Organisation. Monitor bevolkingsonderzoek borstkanker 2018/2019. 2020.

7. De Waard F, Collette HJA, Rombach JJ, Baanders-Van Halewijn EA, Honing C. The dom project for the early detection of breast cancer, Utrecht, The Netherlands. J Chronic Dis. 1984;37(1):1-44.

8. Verbeek ALM, Holland R, Sturmans F, Hendriks JHCL, Avunac M, Day NE. Reduction of breast cancer mortality through mass screening with modern mammography. First results of the Nijmeger Project, 1975 - 1981. Lancet. 1984;1(8388):1222-4.

9. D’Orsi C, Sickles E, Mendelson E, Morris E, et al. ACR BI-RADS ${ }^{\circledR}$ Atlas, Breast Imaging Reporting and Data System. Am Coll Radiol. 2013;

10. Duijm LEM, Louwman MWJ, Groenewoud JH, van de Poll-Franse L V, Fracheboud J, Coebergh JW. Inter-observer variability in mammography screening and effect of type and number of readers on screening outcome. Br J Cancer. 2009;100(6):901-7.

11. Gur D, Sumkin JH, Hardesty LA, Clearfield RJ, Cohen CS, Ganott MA, et al. Recall and Detection Rates in Screening Mammography: A Review of Clinical Experience - Implications for Practice Guidelines. Cancer. 2004;100(8):1590-4.

12. Klompenhouwer EG, Voogd AC, Den Heeten GJ, Strobbe LJA, De Haan AFJ, Wauters CA, et al. Blinded double reading yields a higher programme sensitivity than non-blinded double reading at digital screening mammography: A prospected population based study in the south of the Netherlands. Eur J Cancer. 2015;51(3):391-9.

13. European Commission. European guidelines for quality assurance in breast cancer screening and diagnosis. Fourth edition. Office for Official Publications of the European Communities. 2013. 138 p. Available from: http://www.euref.org/european-Guidelines

14. Klompenhouwer EG, Voogd AC, den Heeten GJ, Strobbe LJA, Tjan-Heijnen VC, Broeders MJM, et al. Discrepant screening mammography assessments at blinded and non-blinded double reading: impact of arbitration by a third reader on screening outcome. Eur Radiol. 2015;25(10):2821-9.

15. Duijm LEM, Groenewoud JH, Hendriks JHCL, de Koning HJ. Independent Double Reading of Screening Mammograms in the Netherlands: Effect of Arbitration Following Reader Disagreements. Radiology. 2004;231(2):564-70.

16. Klompenhouwer EG, Weber RJP, Voogd AC, den Heeten GJ, Strobbe LJA, Broeders MJM, et al. Arbitration of discrepant BI-RADS 0 recalls by a third reader at screening mammography lowers recall rate but not the cancer detection rate and sensitivity at blinded and non-blinded double reading. Breast. 2015;24(5):601-7.

17. Ciatto S, Ambrogetti D, Risso G, Catarzi S, Morrone D, Mantellini P, et al. The role of arbitration of discordant reports at double reading of screening mammograms. J Med Screen. 2005;12(3):125-7.

18. Caumo F, Brunelli S, Tosi E, Teggi S, Bovo C, Bonavina G, et al. On the role of arbitration of discordant double readings of screening mammography: experience from two Italian programmes. Radiol Med. 2011;116(1):84-91. 
19. Healy NA, O'Brien A, Knox M, Hargaden G, Smith C, Fenlon H, et al. Consensus review of discordant imaging findings after the introduction of digital screening mammography: Irish national breast cancer screening program experience. Radiology. 2020;295(1):35-41.

20. Duijm LEM, Groenewoud JH, Fracheboud J, de Koning HJ. Additional double reading of screening mammograms by radiologic technologists: Impact on screening performance parameters. J Natl Cancer Inst. 2007;99(15):1162-70.

21. Duijm LEM, Groenewoud JH, Fracheboud J, van Ineveld BM, Roumen RMH, de Koning HJ. Introduction of additional double reading of mammograms by radiographers: Effects on a biennial screening programme outcome. Eur J Cancer. 2008;44(9):1223-8.

22. Tonita JM, Hillis JP, Lim C-H. Medical Radiologic Technologist Review: Effects on a Population-based Breast Cancer Screening Program. Radiology. 1999;211(2):529-33.

23. Torres-Mejía G, Smith RA, Carranza-Flores M de la L, Bogart A, Martínez-Matsushita L, Miglioretti DL, et al. Radiographers supporting radiologists in the interpretation of screening mammography: a viable strategy to meet the shortage in the number of radiologists. BMC Cancer. 2015;15(1):410.

24. Pauli R, Hammond S, Cooke J, Ansell J. Comparison of radiographer/radiologist double film reading with single reading in breast cancer screening. J Med Screen. 1996;3(1):18-22.

25. Setz-Pels W, Duijm LEM, Groenewoud JH, Voogd AC, Jansen FH, Hooijen MJHH, et al. Detection of bilateral breast cancer at biennial screening mammography in the Netherlands: a population-based study. Radiology. 2011;260(2):357-63.

26. Wang SY, Long JB, Killelea BK, Evans SB, Roberts KB, Silber A, et al. Preoperative breast magnetic resonance imaging and contralateral breast cancer occurrence among older women with breast cancer. J Clin Oncol. 2016;34(4):321-8.

27. El Sharouni MA, Postma EL, Menezes GLG, van den Bosch MAAJ, Pijnappel RM, Witkamp AJ, et al. High Prevalence of MRI-Detected Contralateral and Ipsilateral Malignant Findings in Patients With Invasive Ductolobular Breast Cancer: Impact on Surgical Management. Clin Breast Cancer. 2016; 16(4):269-75. 


\section{Chapter 2}

Impact of the second reader on screening outcome at blinded double reading of digital screening mammograms

Angela M.P. Coolen, Adri C. Voogd, Luc J. Strobbe, Marieke W.J. Louwman, Vivianne C.G. Tjan-Heijnen, Lucien E.M. Duijm British Journal of Cancer 2018;119(4):503-507 


\section{Abstract}

\section{Background}

To determine the impact of the second reader on screening outcome at blinded double reading of digital screening mammograms.

\section{Methods}

We included a consecutive series of 99,013 digital screening mammograms, obtained between July 2013 and January 2015 and double read in a blinded fashion. During 2-year follow-up, we collected radiology, surgery and pathology reports of recalled women.

\section{Results}

Single reading resulted in 2928 recalls and 616 screen-detected cancers (SDCs). The second reader recalled another 612 women, resulting in 82 additional SDCs. Addition of the second reader increased the recall rate $(3.0 \%$ to $3.6 \%, p<0.001)$, cancer detection rate $(6.2-7.0$ per 1000 screens, $p<0.001)$ and false positive recall rate $(24.4-28.7$ per 1000 screens, $p<0.001)$. Positive predictive value of recall $(21.0 \%$ vs. $19.7 \%, p=0.20)$ and of biopsy $(52.1 \%$ vs. $50.9 \%, p=0.56)$ were comparable for single reading and blinded double reading. Tumour characteristics were comparable for cancers detected by the first reader and cancers additionally detected by the second reader, except of a more favourable tumour grade in the latter group.

\section{Conclusions}

At blinded double reading, the second reader significantly increases the cancer detection rate, at the expense of an increased recall rate and false positive recall rate. 


\section{Introduction}

The first regional and nationwide breast cancer screening programmes were implemented in the $1980^{\prime}$ s. $^{1}$ These screening programmes aimed to reduce breast cancer mortality through detection and treatment of breast malignancies at an early stage. In the Netherlands, the nationwide biennial breast cancer screening programme was gradually implemented from 1989 through 1997. ${ }^{2}$ Years later, screenfilm mammography (SFM) was replaced by full-field digital mammography (FFDM), significantly enhancing the cancer detection rate $(C D R) \cdot{ }^{3,4}$ In our screening region, the transition to FFDM was completed in 2010. Twenty years after the introduction of biennial mammography screening in the Netherlands, breast cancer mortality has declined by $30-34 \% .^{5}$ The reported breast cancer mortality before the implementation of the Dutch nationwide breast cancer screening programme was 91.6/100,000.

This was reduced to $75.4 / 100,000$ in the SFM period and further declined to $55.1 / 100,000$ in $2014 .^{6}$ This mortality reduction is attributed to the combination of an earlier detection of breast cancer and significant improvements in breast cancer treatment. ${ }^{5}$ Reading strategies used to assess screening mammograms are single reading, with or without computer aided detection (CAD), and double reading. Double reading can be performed in either a non-blinded or blinded fashion. At non-blinded (or 'independent') double reading, the second reader is aware of the first reader's opinion, whereas the second reader is blinded to the opinion of the first reader in case of blinded double reading. In conjunction with European guidelines, ${ }^{7}$ double reading is standard of care in the Dutch nationwide breast cancer screening programme. Studies dating from the era of SFM have shown that non-blinded double reading significantly increases the CDR compared to single reading. ${ }^{8,9}$ With the introduction of digital mammography, blinded double reading became technically feasible. Klompenhouwer et al. compared blinded and non-blinded double reading and found a higher CDR and programme sensitivity at blinded double reading, at the expense of an increased recall rate and false positive recall rate. ${ }^{10}$ In the current study we prospectively determined screening outcome at blinded double reading vs. single reading in a biennial digital screening mammography programme in a southern region of the Netherlands. 


\section{Materials and methods}

\section{Study population}

In this prospective study we used information from 99,013 consecutive screening examinations (9860 initial screens and 89,143 subsequent screens, respectively) performed between 1 July 2013 and 1 January 2015. These FFDM were obtained at four specialised screening units in a southern biennial screening mammography region of the Netherlands (BOZ, Bevolkings Onderzoek Zuid). The Dutch breast cancer screening programme targets women aged 50-75. On entering the Dutch nationwide screening programme, all women are routinely asked to give permission to use their data for scientific purposes. One woman refused this permission and was therefore excluded from our study. This study was performed within the national permit for breast cancer screening and did not require an additional permit, according to the Dutch Law on Population-based screening.

\section{Screening procedure and recall}

Details of the Dutch nationwide breast cancer screening programme have been described previously. ${ }^{11}$ In brief, mammograms were acquired by certified technologists using a Lorad Selenia FFDM system (Hologic Inc, Danbury, CT), with a 70 $\mu \mathrm{m}$ pixel size and a $232 \times 286 \mathrm{~mm}$ field of view.

All 99,013 mammographic examinations were double read in a blinded fashion by a team of 13 certified screening radiologists, each of them reading at least 10,000 mammograms per year. Thus, when the first reader decided to recall, the mammogram was always read by a second screening radiologist. Mammograms were classified according to the Breast Imaging Reporting and Data System (BI-RADS). ${ }^{12}$ Previously obtained mammograms were available for comparison in case of a subsequent screening. Although in the Dutch screening programme, after performing the mammographic examination, the technologist annotates whether she would recall a women, technologists do not function as official readers. The radiologists were not blinded to the technologists opinion.

Mammographic abnormalities were classified as either a suspicious mass, suspicious microcalcifications, suspicious mass with microcalcifications, architectural distortion, asymmetry or other abnormality. A discordant reading was defined as a difference in classification by two readers, where one reader classified the mammogram BI-RADS 1 or 2 (negative, i.e. no recall) and the other reader classified it as either BI-RADS 0,4 or 5 (positive, i.e. recall). All other cases were classified as concordant readings. In addition to all concordant BI-RADS 0, 4 and 5 mammograms, 
all discordant readings were recalled (i.e. no arbitration took place for discordant readings).

For training purposes, every six weeks, a supervising breast radiologist discussed all recall decisions made by the screening radiologists, with the technologists. In addition, all cases with a negative reading at radiologist blinded double reading but a positive technologist reading were re-assessed by the supervising breast radiologist. $A$ woman was recalled at this stage if the supervising radiologist considered workup necessary.

\section{Diagnostic workup and follow-up after recall}

In case of a positive screening result, the woman was referred by her general practitioner to the breast unit of one of the nearby hospitals. The general practitioner and the hospital breast unit were only informed about the type of mammographic abnormality and corresponding BI-RADS classification and were blinded to the type of recall (discordant vs. concordant). After physical examination by a surgical oncologist or dedicated breast nurse, additional mammographic and/or tomosynthesis views were obtained at the clinical radiologist's discretion. The previous screening mammograms were routinely available and stored in the Picture Archiving and Communication System of the hospital. All mammograms were classified according to BI-RADS. Dependent on the outcome of both the physical examination and mammography, further workup consisted of one or a combination of the following: breast ultrasonography (US), MR imaging and/or biopsy. During a follow-up period of about 2 years (until the next biennial screening examination) screening mammography findings, clinical data, additional clinical imaging reports, biopsy reports and surgery reports were collected of all recalled women. Breast cancers were divided in ductal carcinoma in situ (DCIS) and invasive cancers. Lobular carcinoma in situ (LCIS) was considered to be a benign lesion.

\section{Statistical analysis}

The primary outcome measures of our study were recall rate (recalls per 100 screens), CDR (CDR, screen-detected cancers per 1000 screens), interval cancer rate (ICR, interval cancers per 1000 screens), false positive rate (FPR, false positive recalls per 1000 screens), positive predictive value (PPV) of recall and PPV of biopsy. These outcome measures were compared for single reading and blinded double reading using McNemar's test (recall rate, CDR and FPR) and Chi-square test (PPV of recall and biopsy). A Chi-square test was performed to test differences in type of mammographic abnormality, BI-RADS classification at recall, diagnosis after recall (true positive vs. false positive), type of screen-detected cancer (DCIS vs. invasive cancer) and also 
tumour stage and other tumour characteristics (size, histopathologic type and grading, hormone receptor status, lymph node status) of tumours detected by single reading and blinded double reading. In case of bilateral disease the tumour with the most advanced tumour stage was included in the analysis. When multiple foci of cancer were found in the same breast, only the largest tumour was taken into account for analysis. The significance level was set at $5 \%$. Statistical analysis was performed using IBM SPSS Statistics 23.0 (IBM SPSS Statistics for Windows, Version 23.0. Armonk, NY: IBM Corp).

\section{Results}

\section{Overall screening results}

Out of 99,013 screened women, 3562 were recalled for further evaluation of a mammographic abnormality (recall rate, 3.6\%). Breast cancer was diagnosed in 704 women, resulting in an overall CDR of 7.1 per 1000 screens and a PPV of recall of 19.8\% (Figure 2.1). The false positive recall rate was 28.9 per 1000 screens $(2858 / 99,013)$. We traced a total of 162 interval cancers, resulting in an interval cancer rate of 1.6 per 1000 screens. Programme sensitivity was 81\% (704/866). Twenty-two women were recalled after reassessment of positive technologist findings, resulting in 6 additional screen-detected cancers. Since the current study focusses on radiologist blinded double reading, these 22 recalls and 6 additional cancers were hereafter excluded from statistical analysis.

\section{Single reading vs. blinded double reading}

The first radiologist recalled a total of 2928/99,013 women (recall rate 3.0\%). Another $612 / 99,013$ women were recalled by the second radiologist, resulting in a significantly higher recall rate at blinded double reading compared to single reading $(3.6 \% \mathrm{vs}$. $3.0 \%, p<0.001$, Table 2.1). The CDR also significantly increased from $6.2(616 / 99,013)$ per 1000 screens at single reading to $7.0(698 / 99,013)$ at blinded double reading $(p<0.001)$, with a $13.3 \%$ relative increase in CDR. The false positive rate per 1000 screens increased from $23.4(2312 / 99,013)$ at single reading to $28.7(2842 / 99,013)$ at blinded double reading $(p<0.001)$. The positive predictive value of recall $(21.0 \%$ $(616 / 2928)$ vs. $19.7 \%(698 / 3540), p=0.20)$ and positive predictive value of biopsy $(52.1 \%(616 / 1128)$ vs. $50.9 \%(698 / 1372), p=0.56)$ were comparable for single reading and blinded double reading. 


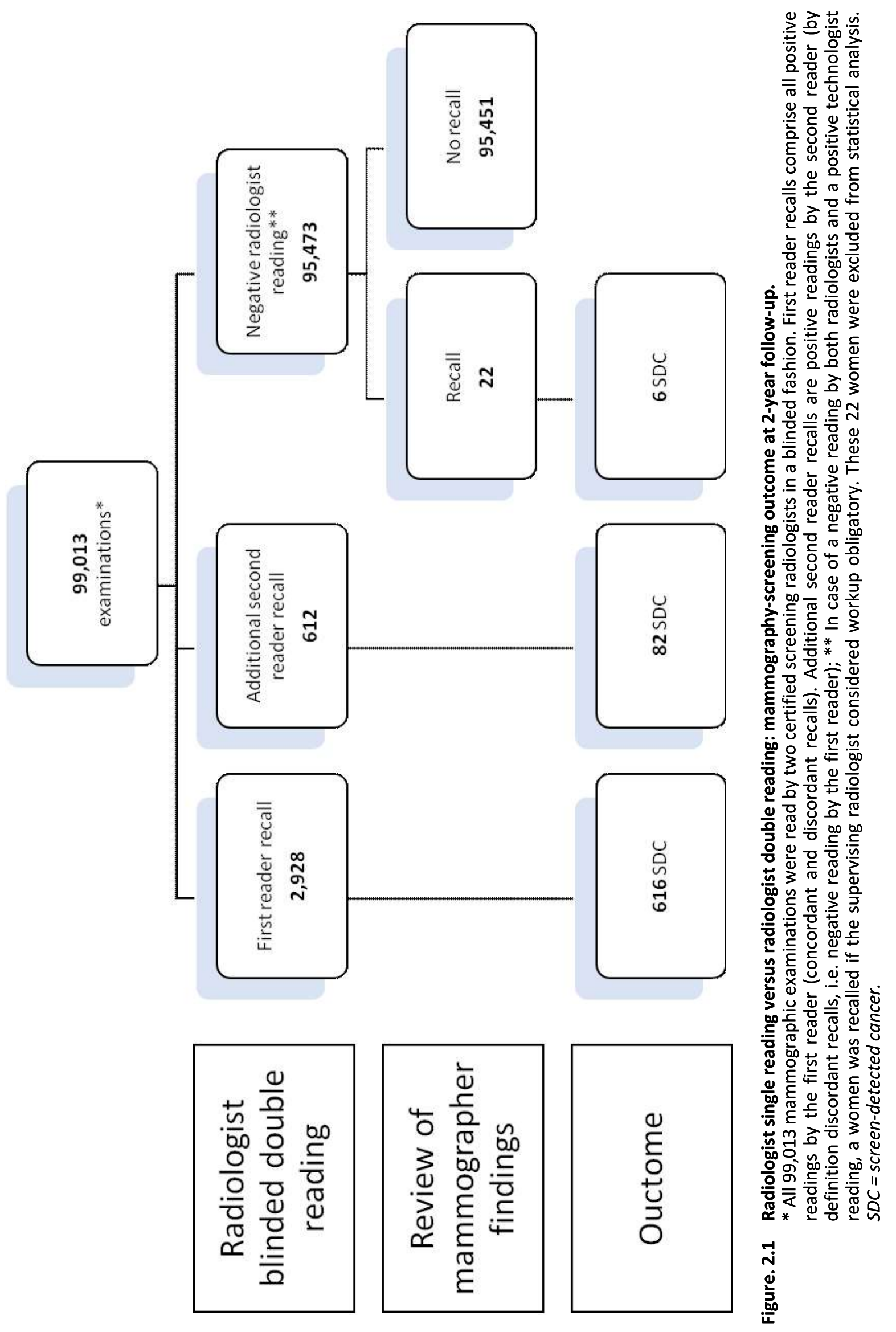


The proportion of BI-RADS 0 lesions was significantly higher in women additionally recalled by the second reader $(74.5 \%$ vs. $62.2 \%, \mathrm{p}<0.001)$, in combination with significantly fewer BI-RADS 4 and 5 recalls (25.5\% vs. $37.8 \%, p<0.001)$ (Table 2.2). The lesions additionally recalled by the second reader comprised larger proportions of asymmetries and architectural distortions than masses and/or microcalcifications when compared to the proportions of various mammographic abnormalities recalled by the first reader ( $p<0.001$, Table 2.2 ). Moreover, for lesions additionally recalled by the second reader, the type of assessment more frequently consisted of breast imaging only, without biopsy ( $p=0.001$, Table 2.2 ). Finally, these lesions comprised more false positive recalls and fewer cancers compared to those lesions recalled by the first reader $(p<0.001$, Table 2.2$)$.

Table 2.1 Screening outcome at single reading versus blinded double reading.

\begin{tabular}{lccc}
\hline & Single reading & Blinded double reading & P-value \\
\hline Recalls, No (recall rate) & $2928(3.0)$ & $3540(3.6)$ & $<0.001$ \\
Screendetected cancers, No (CDR) & $616(6.2)$ & $698(7.0)$ & $<0.001$ \\
False positives, No (FPR) & $2312(23.4)$ & $2842(28.7)$ & $<0.001$ \\
Positive predictive value of recall, \% & 21.0 & 19.7 & 0.20 \\
Positive predictive value of biopsy, \% & 52.1 & 50.9 & 0.56 \\
\hline
\end{tabular}

$C D R=$ Cancer Detection Rate (number of cancers detected per 1000 screens); FPR = False positive rate (number of false positive recalls per 1000 screens).

Table 2.2 Mammographic characteristics and outcome of lesions recalled by the $\mathbf{1}^{\text {st }}$ reader versus lesions additionally recalled by the $2^{\text {nd }}$ reader.

\begin{tabular}{|c|c|c|c|}
\hline & $\begin{array}{c}\text { Recalls by } 1^{\text {st }} \text { reader } \\
(n=2,928)\end{array}$ & $\begin{array}{c}\text { Recalls by } 2^{\text {nd }} \text { reader } \\
(n=612)\end{array}$ & P-value \\
\hline \multicolumn{4}{|l|}{ Mammographic abnormality* } \\
\hline Mass & $2084(71.2)$ & $427(69.8)$ & $<0.001$ \\
\hline Microcalcifications & $429(14.7)$ & $75(12.3)$ & \\
\hline Mass with microcalcifications & $106(3.6)$ & $14(2.3)$ & \\
\hline Asymmetry & $119(4.1)$ & $29(4.7)$ & \\
\hline Architectural distortion & $190(6.5)$ & 67 (10.9) & \\
\hline \multicolumn{4}{|l|}{ BI-RADS at recall } \\
\hline BI-RADS 0 & $1820(62.2)$ & $285(75.4)$ & $<0.001$ \\
\hline BI-RADS 4 or 5 & $1108(37.8)$ & $93(24.6)$ & \\
\hline \multicolumn{4}{|l|}{ Type of assessment after recall } \\
\hline None & $10(0.3)$ & $0(0)$ & 0.001 \\
\hline Clinical breast imaging & $1736(59.3)$ & $422(69.0)$ & \\
\hline Clinical breast imaging + biopsy & $1182(40.4)$ & $190(31.0)$ & \\
\hline \multicolumn{4}{|l|}{ Screening outcome } \\
\hline False positive & $2312(79.0)$ & $530(86.6)$ & $<0.001$ \\
\hline True positive & $616(21.0)$ & $82(13.4)$ & \\
\hline
\end{tabular}

*Dominant mammographic abnormality in case of multiple recalled lesions 


\section{Tumour characteristics of screen-detected cancers}

The tumour characteristics of cancers detected by the first reader were comparable to those of cancers additionally detected by the second reader, except of the tumour grade of invasive cancers (Table 2.3). The proportion of well differentiated, invasive cancers (Bloom \& Richardson grade I) was larger in the group of 61 invasive cancers additionally detected by the second reader (59.0\% vs. $39.8 \%, p=0.021$, Table 2.3 ). The final surgical treatment (breast conserving surgery vs. mastectomy) also did not differ significantly between both groups (Table 2.3).

\section{Discussion}

The current study reports on the impact of the second reader on screening outcome at blinded double reading of screening mammograms in a southern region of the Dutch nationwide breast cancer screening programme. Compared to single reading, addition of a second reader significantly increased the CDR, at the expense of a significantly increased recall rate and false positive recall rate.

Breast screening programmes aim to reduce patient morbidity and mortality through the detection of early-stage breast cancers, with acceptable false positive recall rates. This balance between $C D R$ and false positive recall rate is a very delicate one. ${ }^{10}$ Compared to single reading, addition of the second reader significantly increased the CDR from 6.2 to 7.0 per 1000 screens, at the expense of a significant increase in recall rate, from $3.0 \%$ to $3.6 \%$. The higher recall rate at blinded double reading, observed in our study, is in line with studies reported by Posso et al. ${ }^{13,14}$ However, these authors found a comparable CDR of 4.2-4.8 and 4.6-5.2 per 1000 screens at single reading and double reading. The significant increase in CDR that we observed after second reading may be largely explained by a difference in screening programme design but also by the fact that we included more screens. Posso et al. included women aged 50-69 years and performed consensus with arbitration of discordant readings. Moreover, their study was characterised by higher recall rates (4.6-4.8\%), much lower CDRs and a PPV of recall of less than $10 \%$. Our recall rate of $3.6 \%$ is still within the ranges of the European guidelines for quality assurance ${ }^{7}$ and the Dutch optimisation study of Otten et al. ${ }^{15}$ 
Table 2.3 Tumour characteristics of cancers detected by the 1st reader and of cancers additionally detected by the 2 nd reader at blinded double reading.

\begin{tabular}{|c|c|c|c|}
\hline & $\begin{array}{c}\text { Cancers detected by } 1^{\text {st }} \\
\text { reader }(n=616)\end{array}$ & $\begin{array}{c}\text { Cancers detected by } 2^{\text {nd }} \\
\text { reader }(n=82)\end{array}$ & P-value \\
\hline \multicolumn{4}{|l|}{ Mammographic abnormality } \\
\hline Mass & $388(63.0)$ & $39(47.6)$ & 0.113 \\
\hline Microcalcifications & 129 (20.9) & $28(34.1)$ & \\
\hline Mass with microcalcifications & $52(8.4)$ & $6(7.3)$ & \\
\hline Asymmetry & $7(1.1)$ & $1(1.2)$ & \\
\hline Architectural distortion & $40(6.5)$ & $8(9.8)$ & \\
\hline \multicolumn{4}{|l|}{ Type of cancer } \\
\hline DCIS* & $108(17.5)$ & $21(25.6)$ & 0.110 \\
\hline Invasive & $508(82.5)$ & $61(74.4)$ & \\
\hline \multicolumn{4}{|l|}{ DCIS* grade } \\
\hline Low & $17(15.7)$ & $5(23.8)$ & 0.616 \\
\hline Intermediate & $39(36.1)$ & $9(42.9)$ & \\
\hline High & $52(48.1)$ & $7(33.3)$ & \\
\hline \multicolumn{4}{|l|}{ Histology of invasive cancers } \\
\hline Ductal & $407(80.1)$ & $47(77.0)$ & 0.368 \\
\hline Lobular & $54(10.6)$ & $4(6.6)$ & \\
\hline Mixed ductal/lobular & $15(3.0)$ & $4(6.6)$ & \\
\hline Other & $32(6.3)$ & $6(9.8)$ & \\
\hline \multicolumn{4}{|l|}{ Tumour size of invasive cancers } \\
\hline $\mathrm{T} 1(\leq 20 \mathrm{~mm})$ & $404(79.5)$ & $53(86.9)$ & 0.103 \\
\hline $\mathrm{T} 2+(>20 \mathrm{~mm})$ & $104(20.5)$ & $8(13.1)$ & \\
\hline \multicolumn{4}{|c|}{ Lymph node status of invasive cancers } \\
\hline $\mathrm{N}+$ & $117(23.0)$ & $11(18.0)$ & 0.324 \\
\hline $\mathrm{N}-$ & $383(75.4)$ & $48(78.7)$ & \\
\hline Unknown & $8(1.6)$ & $2(3.3)$ & \\
\hline \multicolumn{4}{|l|}{ Bloom \& Richardson grade } \\
\hline I & $202(39.8)$ & $36(59.0)$ & 0.021 \\
\hline II & $236(46.5)$ & $16(26.2)$ & \\
\hline III & $63(12.4)$ & $9(14.8)$ & \\
\hline Unknown & $7(1.4)$ & 0 & \\
\hline \multicolumn{4}{|l|}{ Estrogen receptor status } \\
\hline Positive & $460(90.6$ & $56(91.8)$ & 0.819 \\
\hline Negative & $45(8.9)$ & $5(8.2)$ & \\
\hline Unknown & $3(0.6)$ & 0 & \\
\hline \multicolumn{4}{|l|}{ Progesterone receptor status } \\
\hline Positive & $369(72.6)$ & $39(63.9)$ & 0.378 \\
\hline Negative & $136(26.8)$ & $22(36.1)$ & \\
\hline Unknown & $6(1.2)$ & 0 & \\
\hline \multicolumn{4}{|l|}{ Her2/Neu receptor status } \\
\hline Positive & $44(8.7)$ & $8(13.1)$ & 0.292 \\
\hline Negative & $458(90.2)$ & $53(86.9)$ & \\
\hline Unknown & $6(1.2)$ & 0 & \\
\hline \multicolumn{4}{|l|}{ Final surgical treatment } \\
\hline Breast conserving surgery & $494(80.2)$ & $61(74.4)$ & 0.534 \\
\hline Mastectomy & $114(18.5)$ & $20(24.4)$ & \\
\hline No surgery & $8(1.3)$ & $1(1.2$ & \\
\hline
\end{tabular}

${ }^{*} D C I S=$ Ductal carcinoma in situ. 
A majority of the 82 cancers additionally detected by the second reader ( $11.7 \%$ of all cancers detected by the screening radiologists) were small ( $\mathrm{T} 1 \mathrm{a}-\mathrm{C})$ invasive cancers $(64.6 \%)$ or DCIS (25.6\%), and most invasive cancers were of low histological grade. As blinded double reading detects additional cancers at an early stage, one may assume that this reading strategy will further reduce breast cancer morbidity and mortality. However, recent studies suggest that some of these small invasive cancers with favourable biological behaviour do not progress to clinically significant cancers during the lifetime of the patient and therefore actually represent overdiagnosis. ${ }^{16,17}$

There were significantly more BI-RADS 0 recalls among women additionally recalled by the second reader than among women recalled after single reading, with a larger proportion of women who underwent imaging only after recall by the second reader. In the Dutch screening setting, BI-RADS 0 represents a mammographic finding needing additional workup and it is generally considered to be a lesion with a relatively low malignancy risk, of $\sim 7 \% .{ }^{18}$ In the Netherlands, nearly $70 \%$ of women with a BI-RADS 0 recall undergo non-invasive assessment only to confirm the benign nature of their abnormality detected at screening mammography, without the need of any additional biopsy procedures. ${ }^{19} \mathrm{~A}$ possible explanation for the differences in recall BI-RADS would be that the percentage of BI-RADS 0 is higher in case of a discordant reading because two readers are more likely to agree on a more obvious (BI-RADS 4 or 5) abnormality. With our study design, additional recalls at blinded double reading are by definition discordant readings. The second reader might however not agree with all readings by the first reader (i.e. there are also discordant readings in the group women recalled by the first reader). Differences in screening outcome and tumour characteristics between discordant vs. concordant recalls at blinded double reading are a subject of further study.

Unlike Posso et al. we did not perform a cost-effectiveness analysis. In 2014, the total cost per screening examination was $€ 66^{6}$ and only a small proportion (less than $10 \%)$ of these costs were spent on the screening radiologists. In the Dutch breast cancer screening programme it is therefore not likely that a change of reading strategy from double to single reading would result in a significant cost reduction. Since Posso et al. did not find a significant increase in CDR with double reading, it is not surprising that they concluded that double reading is not a cost-effective strategy.

Our study has several strengths and limitations. It is a large study, with virtually complete follow-up, that focuses on the impact of a second reader on screening outcome. On the other hand, as mentioned earlier, our report does not provide information on the cost-effectiveness of blinded double reading, a topic that is of importance for further study in order to make a definite recommendation. Second, extrapolation of our results to other screening programmes may be limited by the fact 
that the design of the Dutch breast cancer screening programme (blinded double reading) and workup strategies differ from other countries. Third, our study design does not allow comparison of interval cancer rates between single and blinded double reading. Another limitation of our study design is that we cannot be sure screening radiologists would read in the same way when placed in a situation where there is no safety net of a second reader. Finally, we did not investigate the influence of arbitration of discordant readings on screening outcome.

In conclusion, we favour blinded double reading over single reading as the first reading strategy significantly increases the $C D R$, at the expense of an acceptable increase in recall rate and false positive recall rate. Further research on costeffectiveness is needed to make a more definite recommendation. 


\section{References}

1. Dowling EC, Klabunde C, Patnick J, Ballard-Barbash R. Breast and cervical cancer screening programme implementation in 16 countries. J. Med Screen. 2010;17:139-146.

2. Duijm LEM, Groenewoud JH, Fracheboud J, de Koning HJ. Additional double reading of screening mammograms by radiologic technologists: Impact on screening performance parameters. J. Natl. Cancer Inst. 2007;99:1162-1170.

3. Bluekens AMJ, Holland R, Karssemeijer N, Broeders MJM, den Heeten GJ. Comparison of digital screening mammography and screen-film mammography in the early detection of clinically relevant cancers: a multicenter study. Radiology 2012;265:707-714.

4. Nederend J, et al. Impact of transition from analog screening mammography to digital screening mammography on screening outcome in The Netherlands: a population-based study. Ann. Oncol. 2012;23:3098-3103.

5. Sankatsing VDV. et al. The effect of population-based mammography screening in Dutch municipalities on breast cancer mortality: 20 years of followup. Int J Cancer 141(4), 671-677 (2017).

6. National Evaluation Team for Breast cancer screening in the Netherlands (NETB), Monitor 2014. 2016:1-6.

7. Perry N, Broeders M, de Wolf C, et al. European Commission. European Guidelines for Quality Assurance in Breast Cancer Screening and Diagnosis, 2006, 4th edition, Luxembourg, Office for Official Publications of the European Communities.

8. Duijm LEM, et al. Inter-observer variability in mammography screening and effect of type and number of readers on screening outcome. Br J Cancer 2009;100:901-907.

9. Gur D, et al. Recall and detection rates in screening mammography: a review of clinical experienceimplications for practice guidelines. Cancer 2004;100:1590-1594.

10. Klompenhouwer EG. et al. Blinded double reading yields a higher programme sensitivity than nonblinded double reading at digital screening mammography: a prospected population based study in the south of the Netherlands. Eur J Cancer 2015;51:391-399.

11. Duijm LEM, Groenewoud JH, Hendriks JHCL, de Koning HJ. Independent double reading of screening mammograms in the netherlands: effect of arbitration following reader disagreements. Radiology 2004;231:564-570.

12. D'Orsi C, et al. ACR BI-RADS ${ }^{\circledR}$ Atlas, Breast Imaging Reporting and Data System. (American College of Radiology: Reston, VA, 2013.

13. Posso MC, Puig T, Quintana MJ, Solà-Roca J, Bonfill X. Double versus single reading of mammograms in a breast cancer screening programme: a costconsequence analysis. Eur. Radiol. 2016;26:32623271.

14. Posso $M$, Carles $M$, Rué $M$, Puig $T$, Bonfill X. Cost-effectiveness of double reading versus single reading of mammograms in a breast cancer screening programme. PLoS One 2016;11:e0159806.

15. Otten JDM, et al. Effect of recall rate on earlier screen detection of breast cancers based on the Dutch performance indicators. J Natl Cancer Inst. 2005;97:748-754.

16. Welch HG, Prorok PC, O'Malley AJ, Kramer BS. Breast-cancer tumor size, overdiagnosis, and mammography screening effectiveness. N Engl J Med 2016;375:1438-1447.

17. Lannin DR, Wang S. Are small breast cancers good because they are small or small because they are good? N Engl J Med. 2017;376:2286-2291.

18. Klompenhouwer EG, et al. Arbitration of discrepant BI-RADS 0 recalls by a third reader at screening mammography lowers recall rate but not the cancer detection rate and sensitivity at blinded and nonblinded double reading. Breast 2015;24:601-607.

19. Timmers JM, et al. Cost-effectiveness of non-invasive assessment in the Dutch breast cancer screening program versus usual care: a randomized controlled trial. Can J Public Heal. 2014;105: e342-e347. 



\section{Chapter 3}

Characteristics of screen-detected cancers following concordant or discordant recalls at blinded double reading in biennial digital screening mammography

Angela M.P. Coolen, Joost R.C. Lameijer, Adri C. Voogd, Marieke W.J. Louwman, Luc J. Strobbe, Vivianne C.G. Tjan-Heijnen, Lucien E.M. Duijm European Radiology 2019;29(1):337-344 


\section{Abstract}

\section{Objectives}

To analyse which mammographic and tumour characteristics led to concordant versus discordant recalls at blinded double reading to further optimise our breast cancer screening programme.

\section{Methods}

We included a consecutive series of 99,013 screening mammograms obtained between July 2013 and January 2015. All mammograms were double read in a blinded fashion. Discordant readings were routinely recalled without consensus or arbitration. During the 2-year follow-up, relevant data of the recalled women were collected. We compared mammographic characteristics, screening outcome and tumour characteristics between concordant and discordant recalls.

\section{Results}

There were 2543 concordant recalls $(71.4 \%)$ and 997 discordant recalls $(28.0 \%)$. The positive predictive value of a concordant recall was significantly higher $(23.5 \%$ vs. $10.0 \%, p<0.001)$. The proportion of BI-RADS 0 was significantly higher in the discordant recall group $(75.7 \%$ vs. $56.3 \%$, $\mathrm{p}<0.001)$. Discordant recalls were more often an asymmetry or architectural distortion $(21.8 \%$ vs. $13.2 \%$ and $9.3 \%$ vs. $6.5 \%$, respectively, $\mathrm{p}<0.001$ ). There were no differences in the distribution of DCIS and invasive cancers and tumour characteristics were comparable for the two groups, except for a more favourable tumour grade in the discordant recall group $(54.7 \%$ vs. $39.9 \%$ grade I tumours, $p=0.022$ ).

\section{Conclusions}

Screen-detected cancers detected by a discordant reading show a more favourable tumour grade than cancers diagnosed after a concordant recall. The higher proportion of asymmetries and architectural distortions in this group provides a possible target for improving screening programmes by additional training of screening radiologists and the implementation of digital breast tomosynthesis. 


\section{Introduction}

Reading strategies used in breast cancer screening programmes can greatly influence the main performance indicators and have therefore been a subject of research for many years. ${ }^{1-6}$ Breast cancer screening programmes aim to reduce patient morbidity and mortality through detection of early stage breast cancer. Obtaining an optimal balance between the main performance indicators [recall rate, cancer detection rate $(C D R)$ and false-positive recall rate (FPR)] is important to minimise anxiety among the screened population and avoid unnecessary costs. Reading strategies to assess screening mammograms are a potential target to improve this balance.

Possible reading strategies include single reading, with or without computer-aided detection (CAD), and double reading, which can be performed in either a non-blinded (independent) or blinded fashion. At blinded double reading the second reader is not informed about the first reader's opinion. European guidelines consider radiologist double reading as the standard of reference for the assessment of screening mammograms. ${ }^{7}$ With the implementation of full-field digital mammography (FFDM), blinded double reading became technically possible in the Dutch nationwide screening mammography programme. Previous studies have shown that non-blinded double reading significantly increases the cancer detection rate compared with single reading at screen-film mammography (SFM) $)^{6,8}$ and that blinded double reading further increases programme sensitivity compared with non-blinded double reading. ${ }^{4}$

The purpose of the current study was to analyse which mammographic and tumour characteristics led to concordant versus discordant recalls to find strategies to further optimise the screening programme.

\section{Materials and methods}

\section{Study population}

In this prospective study we included 99,013 consecutive screening examinations (9860 initial screens and 89,143 subsequent screens, respectively). These mammograms were performed between July 1, 2013, and January 1, 2015, in a southern screening region of The Netherlands. In this screening region, the transition from screen-film mammography (SFM) to full-field digital mammography (FFDM) was completed in 2010. Full-field digital mammograms (FFDM) were obtained at four specialised screening units. All women entering the Dutch nationwide screening programme are routinely asked to give permission to use their data for evaluation of the screening programme and for scientific purposes. One woman refused this 
permission and was therefore excluded from this study. This study was performed under the national permit for breast cancer screening, which is issued by the Ministry of Health, Welfare and Sports with permission of the Dutch Health Council and did not require additional ethical approval.

\section{Screening procedure and recall}

Details of the Dutch nationwide biennial breast cancer screening programme, which targets asymptomatic women aged 50-75 years, have previously been described. ${ }^{9}$ In brief, all mammographic examinations were performed by certified technologists using a Lorad Selenia FFDM system (Hologic Inc., Danbury, CT) with a 70- $\mu$ m pixel size


technologist annotated whether or not she would recall the woman. This is routine practice in our nationwide screening programme. All mammograms were then double read in a blinded fashion by a team of 13 certified screening radiologists. The radiologists were not blinded to the technologist's opinion. In case of a subsequent screening, previously obtained mammograms were always available for comparison. Mammographic abnormalities were classified as a suspicious mass, suspicious microcalcifications, suspicious mass with microcalcifications, architectural distortion, asymmetry or another abnormality. Mammograms were classified according to the Breast Imaging Reporting and Data System (BI-RADS) ${ }^{10}$; the BI-RADS 3 classification is not used in the Dutch screening programme. BI-RADS classification at recall was defined as the highest BI-RADS of two readers. A discordant reading was defined as a difference in classification by two radiologists, where one classified the mammogram as negative (BI-RADS 1 or 2, i.e. no recall) and the other classified it as positive (BIRADS 0,4 or 5 , i.e. recall). All other cases were classified as concordant readings. In addition to all concordant positive screening examinations, all discordant readings were recalled without a consensus meeting between the two radiologists or arbitration by a third reader. For the purpose of quality assurance, every 6 weeks, a supervising breast radiologist discussed all recall decisions with the technologists. All cases that only the radiographers would have recalled were also reviewed. At this stage, a woman was recalled if the supervising radiologist considered work-up necessary.

\section{Diagnostic work-up and follow-up after recall}

In case of a positive screening result, the woman was referred to a hospital breast unit by her general physician. After physical examination by a surgical oncologist or dedicated breast nurse, additional mammographic and/or tomosynthesis views were obtained at the clinical radiologist's discretion and classified according to BI-RADS. Previous screening mammograms were routinely available for comparison via the 
hospital's Picture-Archiving and Communication System (PACS). Dependent on the outcome of the physical examination and clinical mammography, further work-up could consist of one or a combination of the following modalities: breast ultrasonography (US), magnetic resonance imaging (MRI) and/or biopsy [fine-needle aspiration (FNAC), core biopsy, stereotactic biopsy, open surgical biopsy]. During the 2-year follow-up (until the next biennial screening examination), screening mammography findings, clinical data as well as imaging, pathology and surgery reports of all the recalled women were collected. Screen-detected cancers were divided into ductal carcinoma in situ (DCIS) and invasive cancers. Lobular carcinoma in situ (LCIS) was considered a benign lesion.

\section{Statistical analysis}

Recalls were classified as either concordant or discordant. Chi-square and Fisher's exact tests were used to compare these two groups regarding: positive predictive value (PPV) of recall, type of screening examination (initial vs. subsequent), type of mammographic abnormality, BI-RADS classification at recall and diagnosis after recall (true positive vs. false positive). Differences in the proportion of invasive and in situ cancers, tumour grade (using the Nottingham grading system) ${ }^{9}$ and other tumour characteristics of screen-detected cancers were also compared for concordant and discordant recalls using chi-square and Fisher's exact tests. In case of bilateral disease, the tumour with the most advanced tumour stage was included in the analysis. In case of multiple foci of cancer, only the largest tumour was taken into account. The twosided significance level was set at $5 \%$. Statistical analysis was performed using IBM SPSS Statistics 23.0 (IBM SPSS Statistics for Windows, version 23.0, IBM Corp., Armonk, NY).

\section{Results}

\section{Overall screening outcome}

Out of 99,013 screened women, 3562 were recalled for further evaluation of a mammographic abnormality (recall rate 3.6\%) resulting in 704 screen-detected cancers (CDR of 7.1 per 1000 screens and FPR of 28.9 per 1000 screens) (Figure 3.1).

The majority of recalls were based on a concordant reading. Most screens were classified as BI-RADS $0(61.8 \%, 2,186 / 3,540)$. The PPV of BI-RADS 0 recalls was $5.9 \%$, of BI-RADS 4 recalls $34.1 \%$ and of BI-RADS 5 recalls $95.5 \%$ ( $\mathrm{p}<0.001)$. Twenty-two women $(0.6 \%, 22 / 3562)$ were recalled after discussion with the supervising breast radiologist 
as part of quality assurance (Figure 3.1). Since the current study focuses on radiologist blinded double reading, these 22 recalls are hereafter excluded.

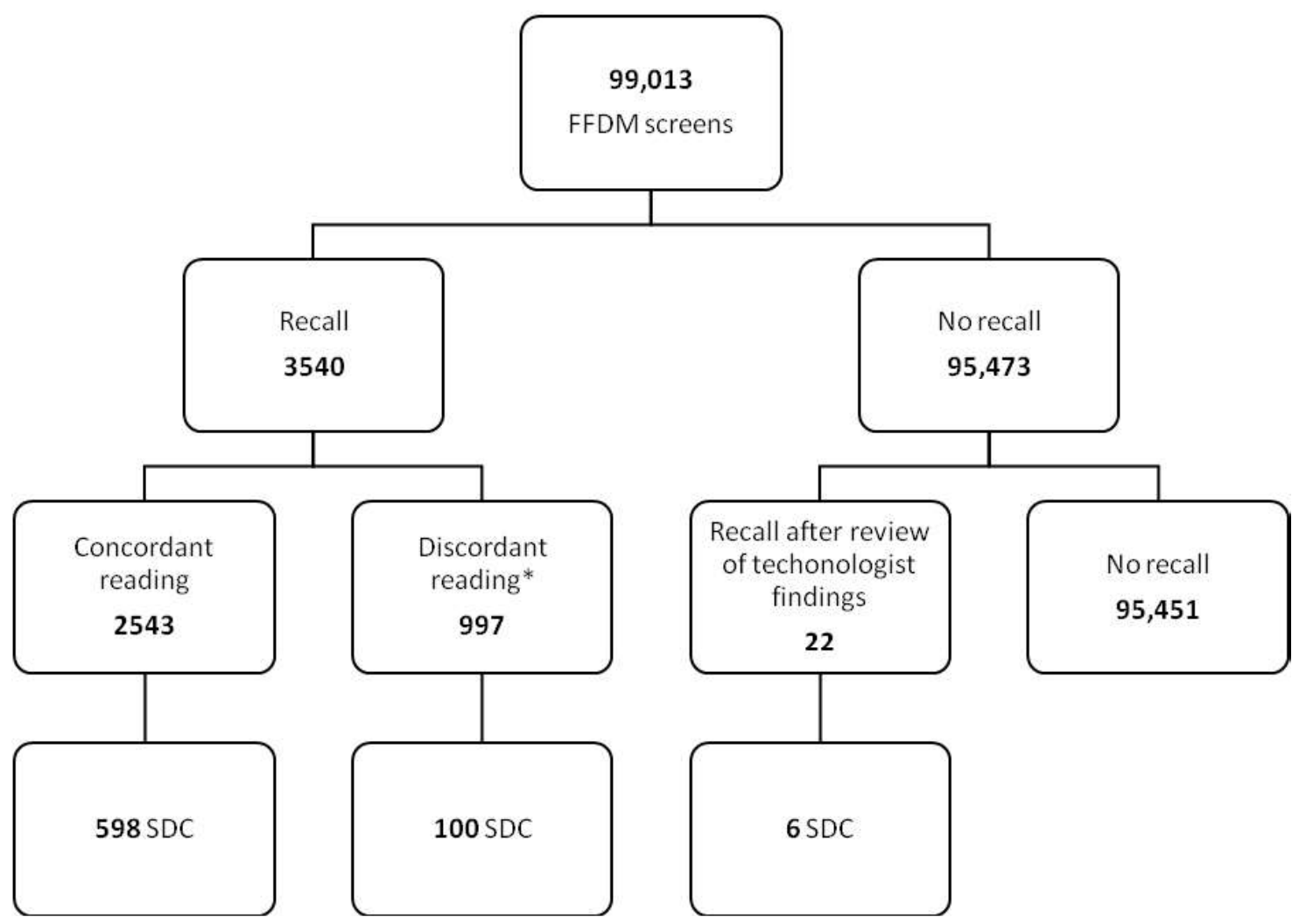

Figure 3.1 Concordant versus discordant recalls at radiologist blinded double reading: screening outcome at 2-year follow-up. FFDM = full-field digital mammography; SDC = screen-detected cancer. * At radiologist blinded double reading, a woman was recalled if the mammogram was considered to be positive by both radiologists or, in case of a discordant reading, if at least one radiologist considered recall necessary. Thus, all discordant readings were recalled, without consensus reading or arbitration being performed.

\section{Mammographic characteristics of concordant versus discordant recalls}

Compared with a concordant reading, a discordant reading was significantly more often an asymmetry or architectural distortion (Table 3.1). BI-RADS classification at recall also differed significantly between the two groups. In the discordant reading group most screens were classified as BI-RADS 0; conversely the percentage of $\mathrm{BI}$ RADS 4 and 5 was higher in the concordant reading group (Table 3.1). The positive predictive value of recall was significantly higher in case of a concordant reading compared with a discordant reading $(23.5 \%$ vs. $10.0 \%$, $p<0.001$, Table 3.1$)$. 


\section{Tumour characteristics of screen-detected cancers detected after concordant versus discordant recalls}

Proportions of DCIS and invasive cancers did not differ significantly and the DCIS grade was comparable for both groups. In particular there was no significant increase in lowgrade DCIS with discordant readings compared with concordant readings $(20.0 \%$ vs. $16.3 \%, p=0.800$, Table 3.2). Tumour characteristics of invasive cancers were comparable for tumours detected by concordant and discordant readings, except for a more favourable tumour grade of invasive cancers detected by discordant reading ( $54.7 \%$ vs. $39.9 \%$ grade I tumours, $p=0.022$, Table 3.2 ). Tumour size of invasive cancers tended to be smaller for cancers detected following a discordant reading, with fewer invasive cancers showing lymph node metastases, but these differences were not statistically significant.

Table 3.1 Concordant versus discordant recalls at radiologist blinded double reading: mammographic characteristics and screening outcome.

\begin{tabular}{|c|c|c|c|c|c|}
\hline \multirow{2}{*}{ Screening round, No (\%) } & \multicolumn{2}{|c|}{$\begin{array}{c}\text { Concordant recall } \\
n=2,543\end{array}$} & \multicolumn{2}{|c|}{$\begin{array}{c}\text { Discordant recall } \\
n=997\end{array}$} & \multirow{2}{*}{$\begin{array}{c}\text { P-value } \\
0.143\end{array}$} \\
\hline & & & & & \\
\hline Initial & 602 & $(23.7)$ & 213 & $(21.4)$ & \\
\hline Subsequent & 1941 & (76.3) & 784 & (78.6) & \\
\hline Mammographic abnormality*, No (\%) & & & & & $<0.001$ \\
\hline Mass & 1556 & $(61.2)$ & 548 & $(55.0)$ & \\
\hline Microcalcifications & 389 & (15.3) & 115 & (11.5) & \\
\hline Mass with microcalcifications & 96 & $(3.8)$ & 24 & $(2.4)$ & \\
\hline Asymmetry & 336 & $(13.2)$ & 217 & $(21.8)$ & \\
\hline Architectural distortion & 166 & $(6.5)$ & 93 & $(9.3)$ & \\
\hline Recall BI-RADS, No (\%) & & & & & $<0.001$ \\
\hline BI-RADS 0 & 1431 & $(56.3)$ & 755 & (75.7) & \\
\hline BI-RADS 4 & 940 & (37.0) & 237 & $(23.8)$ & \\
\hline BI-RADS 5 & 172 & $(6.8)$ & 5 & $(0.5)$ & \\
\hline Diagnosis, No (\%) & & & & & $<0.001$ \\
\hline True positive & 598 & (23.5) & 100 & $(10.0)$ & \\
\hline False positive & 1945 & (76.5) & 897 & $(90.0)$ & \\
\hline
\end{tabular}

* Dominant mammographic abnormality in case of multiple recalled lesions. 
Table 3.2 Concordant versus discordant recalls at radiologist blinded double reading: tumour characteristics of screen detected cancers.

\begin{tabular}{|c|c|c|c|c|c|}
\hline \multirow{2}{*}{ Type of cancer, No (\%) } & \multicolumn{2}{|c|}{$\begin{array}{c}\text { Concordant recall } \\
n=598\end{array}$} & \multicolumn{2}{|c|}{$\begin{array}{c}\text { Discordant recall } \\
n=100\end{array}$} & \multirow{2}{*}{\begin{tabular}{|l|} 
P-value \\
0.094
\end{tabular}} \\
\hline & & & & & \\
\hline DCIS* & 104 & $(17.4)$ & 25 & $(25.0)$ & \\
\hline Invasive & 494 & $(82.6)$ & 75 & $(75.0)$ & \\
\hline DCIS grade, No (\%) & & & & & 0.800 \\
\hline Low & 17 & $(16.3)$ & 5 & $(20.0)$ & \\
\hline Intermediate & 38 & (36.5) & 10 & $(40.0)$ & \\
\hline High & 49 & $(47.1)$ & 10 & $(40.0)$ & \\
\hline Histology of invasive cancers, No (\%) & & & & & 0.320 \\
\hline Ductal & 389 & (78.7) & 60 & (80.0) & \\
\hline Lobular & 54 & (10.9) & 4 & $(5.3)$ & \\
\hline Mixed ductal/lobular & 14 & $(2.8)$ & 5 & $(6.7)$ & \\
\hline Other & 33 & (6.7) & 6 & $(8.0)$ & \\
\hline Unknown & 4 & $(0.8)$ & 0 & $(0.0)$ & \\
\hline Tumour size of invasive cancers, No (\%) & & & & & 0.086 \\
\hline $\mathrm{T} 1(\leq 20 \mathrm{~mm})$ & 391 & $(79.1)$ & 66 & $(88.0)$ & \\
\hline $\mathrm{T} 2+(>20 \mathrm{~mm})$ & 103 & (20.9) & 9 & (12.0) & \\
\hline Lymph node status of invasive cancers, No (\%) & & & & & 0.117 \\
\hline $\mathrm{N}+$ & 116 & $(23.5)$ & 12 & $(16.0)$ & \\
\hline $\mathrm{N}-$ & 371 & $(75.1)$ & 60 & $(80.0)$ & \\
\hline $\mathrm{Nx}$ & 7 & $(1.4)$ & 3 & $(4.0)$ & \\
\hline Nottingham grade, No (\%) & & & & & 0.022 \\
\hline 1 & 197 & (39.9) & 41 & $(54.7)$ & \\
\hline II & 230 & $(46.6)$ & 22 & $(29.3)$ & \\
\hline III & 60 & (12.1) & 12 & $(16.0)$ & \\
\hline Unknown & 7 & $(1.4)$ & 0 & $(0.0)$ & \\
\hline Oestrogen receptor status, No (\%) & & & & & 0.785 \\
\hline Positive & 448 & (90.7) & 68 & $(90.7)$ & \\
\hline Negative & 43 & $(8.7)$ & 7 & $(9.3)$ & \\
\hline Unknown & 3 & $(0.6)$ & 0 & $(0.0)$ & \\
\hline Progesterone receptor status, No (\%) & & & & & 0.297 \\
\hline Positive & 359 & $(72.7)$ & 49 & $(65.3)$ & \\
\hline Negative & 132 & $(26.7)$ & 26 & (34.7) & \\
\hline Unknown & 3 & $(0.6)$ & 0 & $(0.0)$ & \\
\hline Her2/Neu receptor status, No (\%) & & & & & 0.263 \\
\hline Positive & 42 & $(8.5)$ & 10 & $(13.3)$ & \\
\hline Negative & 446 & $(90.3)$ & 65 & $(86.7)$ & \\
\hline Unknown & 6 & $(1.2)$ & 0 & $(0.0)$ & \\
\hline Triple negative tumour, No (\%) & & & & & 0.553 \\
\hline Yes & 28 & $(5.7)$ & 3 & $(4.0)$ & \\
\hline No & 466 & $(94.3)$ & 72 & $(96.0)$ & \\
\hline
\end{tabular}

* DCIS = Ductal carcinoma in situ 


\section{Discussion}

To our knowledge, this is the first study comparing screening mammography findings and a wide range of tumour characteristics between concordant and discordant recalls at radiologist blinded double reading in digital mammography screening. We found that a discordant reading between radiologists was present in more than a quarter of all recalls and the majority of these discordant recalls comprised BI-RADS 0 mammographic abnormalities. Screen-detected cancers tended to be more favourable following discordant recalls, expressed in a more favourable tumour grading and a trend to smaller tumours, less often associated with nodal involvement.

The percentage of discordant recalls was lower than the one found in a previous study by Klompenhouwer et al. (28\% vs. $57 \%$, respectively) ${ }^{4}$, set between 2009 and 2011 in the same screening region and population and also using FFDM (with the transition from SFM just completed). This difference might, at least partly, be explained by the slightly higher recall rate (3.6\% vs. $3.3 \%)$ in recent years. Also, screening radiologists have since then gained an additional 2 years of experience reading digital mammograms, are being continuously trained and receive regular feedback on their performance. It is likely that this has improved interobserver agreement and thus lowered the number of discordant recalls. The PPV of discordant recalls was significantly lower compared with concordant recalls. The effect of third reader arbitration of discordant double readings on screening outcome has been documented previously. For example, Ciatto et al. ${ }^{10}$ studied the effect of arbitration of discordant double readings and concluded that it reduces recall rates with a limited reduction in cancer detection rate. A study by Klompenhouwer et al. ${ }^{11}$ showed that arbitration of all discordant readings by a third reader improves the recall rate and increases the PPV of recall, but unfortunately also decreases programme sensitivity. Arbitration of only discordant BI-RADS 0 readings appeared to be a better strategy. ${ }^{12}$

The proportions of DCIS and invasive cancers were comparable for screendetected cancers detected by either concordant or discordant readings. In particular, the percentage of low-grade DCIS, a potential candidate for overdiagnosis ${ }^{13,14}$, did not differ significantly between the two groups. We found that in both groups most screen-detected cancers were small ( $\mathrm{T} 1 \mathrm{a}-\mathrm{C})$ invasive cancers and overall there was a high percentage of oestrogen and progesterone receptor positive tumours. The proportion of triple-negative tumours was very low and comparable in both groups. Recent studies suggest that these small invasive cancers with favourable biological behaviour (mainly hormone receptor-positive tumours) in many cases do not progress to larger, more aggressive cancers during the lifetime of the patient and therefore also represent over-diagnosis. ${ }^{15,16}$ Two of the major harms of overdiagnosis are the over-treatment that results and the anxiety and fear that a cancer diagnosis 
engenders. ${ }^{16}$ It has also been suggested that, compared with other women, breast cancer patients have an increased risk to die from diseases of pulmonary circulation, various external causes and several heart diseases, which is attributed to both breast cancer treatment and breast cancer itself. ${ }^{17}$ Tumour characteristics were comparable for both groups, except for differences in tumour grade; discordant invasive cancers were mostly well differentiated grade I tumours whereas concordant cancers were mostly grade II tumours. However, $45.3 \%$ of invasive cancers detected after a discordant recall were grade II or III cancers and $80 \%$ of DCIS were intermediate and high grade. Therefore, with concordant and discordant recalls, screen-detected cancers appear to represent a mix of overdiagnosis and early detection.

In our study, the majority of recalls were classified as BI-RADS 0 and the proportion of BI-RADS 0 recalls was also significantly higher in the discordant than in the concordant reading group. In the Dutch screening setting, BI-RADS 0 represents a mammographic abnormality requiring further work-up. It is, however, generally considered to be a lesion with a relatively low malignancy risk of approximately $7 \%{ }^{12}$ and the PPV of $5.9 \%$ of a BI-RADS 0 recall found in our study is in line with this report. A possible explanation for the high proportion of BI-RADS 0 recalls in the discordant reading group could be that two readers are more likely to agree on a more obvious (BI-RADS 4 or 5 ) abnormality. ${ }^{18}$ This hypothesis is supported by the fact that the percentage of 'subtle' abnormalities such as asymmetries and, to a lesser degree, architectural distortions was indeed higher in the discordant reading group. Conversely, the proportion of suspicious masses and microcalcifications was higher in the concordant reading group. Various studies have also shown that there is a substantial inter- and intra-observer variability in using the BI-RADS lexicon among radiologists. ${ }^{19,20}$ As the second reader is not informed about the first reader's opinion at blinded double reading, this inter-observer variability is likely to have more of an impact on screening outcome than at non-blinded double reading.

The differences in type of mammographic characteristics provide possible strategies for improvement. In training new and experienced screening radiologists, additional emphasis could be placed on the 'subtle' mammographic abnormalities such as asymmetries and architectural distortions. A recent study by Houssami et al. showed that a single reading of 3D mammography (digital breast tomosynthesis) detected more breast cancers and had a lower FPR compared with the current practice of double-reading 2D mammography alone. ${ }^{21}$ Another recent study by Dibble et al. showed that digital breast tomosynthesis decreases inter-observer variability and increases reader confidence in the detection of architectural distortion. ${ }^{22}$ Previously, Durand et al. showed that use of digital breast tomosynthesis is associated with a lower recall rate of screening mammography, most often for asymmetries. ${ }^{23}$ Digital breast tomosynthesis might be implemented in the Dutch nationwide breast 
cancer screening programme in the future. Preliminary results of artificial intelligence and deep learning for the assessment of mammograms are also promising. ${ }^{24}$ In the future, these computer algorithms may aid the screening radiologist in determining which women should be recalled at screening mammography.

The strength of our study lies in the large study population with virtually complete follow-up and the fact that we provide information on a wide range of tumour characteristics. However, our study also has several limitations. First, the recall rate of the Dutch nationwide breast cancer screening programme is among the lowest worldwide. Data from our study might therefore not necessarily be transposable to other screening programmes. Second, although we did not perform a costeffectiveness analysis, since double reading of screening mammograms is already standard in the Netherlands, blinded double reading is not likely to increase screening costs apart from the higher costs associated with a higher recall rate. Double reading, either non-blinded (independent) or blinded, might however not be deemed cost effective in other screening programmes.

In conclusion, discordant recalls proved to be malignant in $10 \%$ of women and these cancers show a more favourable tumour grade than cancers diagnosed after a concordant recall. Differences in mammographic characteristics, mainly the higher proportion of asymmetries and architectural distortions in the discordant reading group, provide a possible target for improving breast cancer screening programmes, for example, by additional training of screening radiologists and also in the implementation of digital breast tomosynthesis. 


\section{References}

1. Duijm LEM, Groenewoud JH, Fracheboud J, van Ineveld BM, Roumen RMH, de Koning HJ. Introduction of additional double reading of mammograms by radiographers: Effects on a biennial screening programme outcome. Eur J Cancer 2008;44(9):1223-1228.

2. Caumo F, Brunelli S, Tosi E, et al. On the role of arbitration of discordant double readings of screeningmammography: experience from two Italian programmes. Radiol Med 2011;116(1):84-91.

3. Azavedo E, Zackrisson S, Mejàre I, Heibert Arnlind M. Is single reading with computer-aided detection (CAD) as good as double reading in mammography screening? A systematic review. BMC Med Imaging 2012;12(1):22.

4. Klompenhouwer EG, Voogd AC, Den Heeten GJ, et al. Blinded double reading yields a higher programme sensitivity than non-blinded double reading at digital screening mammography: A prospected population based study in the south of the Netherlands. Eur J Cancer 2015;51(3):391-399.

5. Posso MC, Puig T, Quintana MJ, Solá-Roca J, Bonfill X. Double versus single reading of mammograms in a breast cancer screening programme: a cost-consequence analysis. Eur Radiol 2016;26(9):32623271.

6. Duijm LEM, Louwman MWJ, Groenewoud JH, van de Poll-Franse LV, Fracheboud J, Coebergh JW. Inter-observer variability in mammography screening and effect of type and number of readers on screening outcome. Br J Cancer 2009;100(6):901-907.

7. European Commission. European guidelines for quality assurance in breast cancer screening and diagnosis. Fourth edition. Office for Official Publications of the European Communities. 2013;138 p.

8. Gur D, Sumkin JH, Hardesty LA, et al. Recall and detection rates in screening mammography: a review of clinical experience - implications for practice guidelines. Cancer 2004;100(8):1590-1594.

9. Elston CW, Ellis IO. Pathological prognostic factors in breast cancer. I. The value of histological grade in breast cancer: experience from a large study with long-term follow-up. Histopathology 1991;19(5):403-410.

10. Ciatto S, Ambrogetti D, Risso G, et al. The role of arbitration of discordant reports at double reading of screening mammograms. J Med Screen 2005;12(3):125-127.

11. Klompenhouwer EG, Voogd AC, den Heeten GJ, et al. Discrepant screening mammography assessments at blinded and non-blinded double reading: impact of arbitration by a third reader on screening outcome. Eur Radiol 2015;25(10):2821-2829.

12. Klompenhouwer EG, Weber RJP, Voogd AC, et al. Arbitration of discrepant BI-RADS 0 recalls by a third reader at screening mammography lowers recall rate but not the cancer detection rate and sensitivity at blinded and non-blinded double reading. Breast 2015;24(5):601-607.

13. Bluekens AMJ, Holland R, Karssemeijer N, Broeders MJM, den Heeten GJ. Comparison of Digital ScreeningMammography and Screen-Film Mammography in the Early Detection of Clinically Relevant Cancers: A Multicenter Study. Radiology 2012;265(3):707-714.

14. van Luijt PA, Heijnsdijk EAM, Fracheboud J, et al. The distribution of ductal carcinoma in situ (DCIS) grade in 4232 women and its impact on overdiagnosis in breast cancer screening. Breast Cancer Res 2016;18(1):47.

15. Welch HG, Prorok PC, O'Malley AJ, Kramer BS. Breastcancer tumor size, overdiagnosis, and mammography screening effectiveness. N Engl J Med 2016;375(15):1438-1447.

16. Lannin DR,Wang S. Are small breast cancers good because they are small or small because they are good? N Engl J Med 2017;376(23):2286-2291.

17. Riihimäki $M$, Thomsen $H$, Brandt A, Sundquist J, Hemminki K. Death causes in breast cancer patients. Ann Oncol 2012;23(3):604-610.

18. Lee AY, Wisner DJ, Aminololama-Shakeri SJ, et al. Interreader variability in the use of BI-RADS descriptors for suspicious findings on diagnostic mammography: a multi-institution study of 10 academic radiologists. Acad Radiol 2017;24(1):60-66.

19. Ciatto S, Houssami N, Apruzzese A, et al. Reader variability in reporting breast imaging according to BI-RADS $^{\circledR}$ assessment categories (the Florence experience). Breast 2006;15(1):44-51. 
20. Redondo A, Comas M, Macià F, et al. Inter- and intraradiologist variability in the BI-RADS assessment and breast density categories for screening mammograms. Br J Radiol 2012;85:1465-1470.

21. Houssami N, Bernardi D, Pellegrini $M$, et al. Breast cancer detection using single-reading of breast tomosynthesis (3Dmammography) compared to double-reading of 2D-mammography: Evidence from a population-based trial. Cancer Epidemiol 2017;47:94-99.

22. Dibble EH, Lourenco AP, Baird GL, Ward RC, Maynard AS, Mainiero MB. Comparison of digital mammography and digital breast tomosynthesis in the detection of architectural distortion. Eur Radiol 2018;28(1):3-10.

23. DurandMA, HaasBM, Yao $X$, et al. Early clinical experience with digital breast tomosynthesis for screening mammography. Radiology 2015;274(1):85-92.

24. Kooi T, Litjens G, van Ginneken B, Gubern-Mérida A, et al. Large scale deep learning for computer aided detection of mammographic lesions. Med Image Anal 2017;35:303-312. 



\section{Chapter 4}

Value of technologists' reading and training on outcome at screening mammography 



\section{Chapter 4.1}

Incorporation of the technologist's opinion for arbitration of discrepant assessments among radiologists at screening mammography

Angela M.P. Coolen, Joost R.C. Lameijer, Adri C. Voogd, Luc J. Strobbe, Marieke W.J. Louwman, Vivianne C.G. Tjan-Heijnen, Lucien E.M. Duijm Breast Cancer Research and Treatment 2018;171(1):143-149 


\section{Abstract}

\section{Purpose}

We determined whether the addition of the technologist's opinion may be helpful in deciding if discordant readings at blinded double reading should be recalled.

\section{Methods}

A consecutive series of 99,013 digital screening mammograms, obtained between July 2013 and January 2015, were included. All mammograms were first interpreted by a technologist and then double read in a blinded fashion by a team of 13 screening radiologists. All concordant and discordant positive readings among radiologists were recalled.

\section{Results}

Out of 3562 recalls, 998 women were recalled after a discordant reading. Of these women, 337 (33.8\%) had a positive technologist assessment, of which $40(11.9 \%)$ were diagnosed with breast cancer. Sixty women with a negative technologist assessment (60/661, 9.1\%) were diagnosed with breast cancer $(p=0.16)$. Recall rate would have decreased with technologist arbitration (3.6\% vs. $2.9 \%, p<0.001$ ). Cancer detection rate decreased with $8.5 \%$, from $7.1 / 1000$ screens to $6.5 / 1000$ screens $(p=0.10)$. Among women with a positive technologist assessment, the probability of breast cancer was highest in case of suspicious microcalcifications and lowest for suspicious masses $(30.4 \%(17 / 56)$ versus $7.0 \%(16 / 212), p<0.001)$. Breast cancers were diagnosed in all groups of mammographic abnormalities, except in women with a suspicious asymmetry and a negative technologist assessment.

\section{Conclusions}

Assessment by a technologist does not provide a significant discriminating ability in case of a discordant radiologist reading and, taking into account the decrease in cancer detection rate, does not appear to be a suitable arbitration strategy for discordant recalls at blinded double reading. 


\section{Introduction}

Screening mammography programmes aim to reduce breast cancer morbidity and mortality through an early detection of breast cancer. In the Netherlands, breast cancer mortality has declined by $30-34 \%$ since the introduction of biennial screening mammography, twenty years ago. This mortality reduction is due to the combination of an earlier detection of breast cancer and improvements in breast cancer treatment. ${ }^{1}$

In conjunction with European guidelines ${ }^{2}$, double reading is standard of care in the Dutch nationwide breast cancer screening programme. Previous studies have shown that double reading increases the cancer detection rate compared to single reading. ${ }^{3,4}$ However, double reading also generates many discordant assessments among screening radiologists. Several reading strategies have been explored to solve the situation in which only one radiologist considers recall necessary. For example, one may choose to recall all discordant readings or add a third screening radiologist or panel of radiologists to determine whether or not the woman should be recalled. ${ }^{5,6}$

It has been shown that screening mammography technologists, who are trained in reading screening mammograms, may detect breast cancers that are missed by screening radiologists. ${ }^{7,8}$ The value of the technologist's opinion in case of discordant readings among screening radiologists has not been investigated. Therefore, we determined whether the addition of the technologist's opinion may be helpful to decide whether or not discordant readings at double reading should be recalled.

\section{Materials and methods}

\section{Study design and study population}

This is an observational follow-up study of women aged 50-75 who attended a biennial breast cancer screening programme conducted in the south of the Netherlands. We included a consecutive series of 99,013 full-field digital screening mammograms (FFDM) obtained between July 1, 2013 and January 1, 2015. Screening was performed at four specialized units in a southern biennial screening mammography region of the Netherlands.

Women participating in our screening mammography programme were asked for permission to use their data for scientific purposes and for the evaluation of the screening programme. One woman refused this permission and was excluded from analysis. This study was performed within the national permit for breast cancer 
screening and ethical approval was waived, according to the Dutch Law on Population-based screening.

\section{Screening procedure and recall}

Details of our breast cancer screening programme have been described previously. ${ }^{9}$ In summary, mammograms were acquired by certified technologists using a Lorad Selenia FFDM system (Hologic Inc, Danbury, CT), with a $70 \mu \mathrm{m}$ pixel size and a $232 \times$ $286 \mathrm{~mm}$ field of view. After having obtained the screening mammogram, the technologist classified the examination according to the Breast Imaging Reporting and Data System (BI-RADS). ${ }^{10}$ Previous screening examinations were available for comparison in case of a subsequent screening. The technologists annotated any abnormalities for which they deemed recall necessary. Mammographic abnormalities were classified as either a suspicious mass, suspicious microcalcifications, suspicious mass with microcalcifications, architectural distortion, asymmetry or other abnormality. All mammographic examinations were then double read in a blinded fashion by a team of 13 certified screening radiologists, who were not blinded to the technologist's opinion. Each radiologist reads more than 10,000 screening mammograms yearly. A discordant reading between two radiologists was defined as a difference in classification where one reader classified the mammogram BI-RADS 1 or 2 (negative, i.e., no recall) and the other reader classified it as either BI-RADS 0,4 , or 5 (positive, i.e., recall). All other cases were classified as concordant readings. In addition to all concordant BI-RADS 0,4 , and 5 mammograms, all discordant readings were recalled (i.e., no arbitration of discordant readings).

\section{Diagnostic workup and follow-up procedure}

Recalled women were assessed at specialised breast units at one of the 15 regional or university hospitals. BI-RADS 4 and BI-RADS 5 lesions were routinely biopsied, while BI-RADS 3 lesions were either biopsied or followed-up. During a follow-up period of 2 years (until the next biennial screen), we routinely collected data on diagnostic imaging procedures, clinical data, biopsy results, and breast surgery reports of all recalled women.

\section{Training of technologists and quality assurance}

At the start of the study, the mean experience of the 32 mammography technologists in screening mammography was 11.3 years (range, $0.3-21.1$ years) and one recently trained technologist joined the team during the inclusion period. All technologists are certified radiologic technologists who receive an elaborate training at the screening organization in combination with a training at the Dutch Expert Centre for Screening prior to their employment as a screening technologist. Although the technical aspects 
of mammography and evaluation of the images for technical quality are central to their training, technologists are also instructed in breast anatomy and mammographic features of benign and malignant breast conditions. Technologists receive further training at the Expert Centre on a regular basis and frequently attend mammography symposia and conferences.

From the start of our mammography screening in 1995, technologists have been encouraged to look for mammographic abnormalities. Also, since the implementation of digital screening mammography in 2009/2010, the technologists classify each screening examination according to BI-RADS. Every six weeks, technologists attend quality assurance and learning sessions under supervision of a breast radiologist. Together with this radiologist, they review a sample of breast cancer cases that have been detected by the screening radiologists only and they also discuss cases that only the technologists would have recalled. Mammographic abnormalities are compared with pathology outcome, and false-negative cases, occurring as interval cancers, are discussed.

\section{Statistical analysis}

All data were stored into a computerized spreadsheet (Excel; Microsoft, Redmond, WA, USA). Descriptive statistics were performed using Statistical Package for Social Sciences 22 (SPSS Inc. Chicago, IL). Distribution of outcome variables at reading strategies was tested with the Chi-square test. All tests were two-sided, and the significance level was set at $5 \%$.

\section{Results}

\section{Overall screening outcome}

Out of 99,013 screened women, 3562 were recalled for further evaluation of a mammographic abnormality (recall rate, 3.6\%). Breast cancer was diagnosed in 704 women, resulting in an overall cancer detection rate of 7.1 per 1,000 screens and a positive predictive value (PPV) of recall of $19.8 \%$. The false positive recall rate was 28.9 per 1,000 screens $(2858 / 99,013)$. The screen-detected cancers comprised 130 cases of ductal carcinoma in situ (18.5\%) and 574 invasive cancers (81.5\%). Of the invasive cancers, respectively, $223(38.9 \%)$ were stage 1a-b ( $\leq 10 \mathrm{~mm}), 238(41.5 \%)$ stage $1 \mathrm{c}(11-20 \mathrm{~mm})$, and $113(19.7 \%)$ stage $2+(>20 \mathrm{~mm})$. Lymph node metastases were present in 130 (22.6\%) of invasive cancers. The majority of women $(79.5 \%$, $560 / 704)$ underwent breast conserving surgery for their screen-detected cancer. 


\section{Technologist assessments of discordant $\mathrm{BI}$-RADS readings among screening radiologists}

The 3562 recalls comprised 998 discordant readings among the screening radiologists (Figure 4.1.1). The technologists would have recalled 337 (33.8\%) of these women, of which 40 (11.9\%) were diagnosed with breast cancer, whereas of the 661 women who would not have been recalled by the technologists 60 (9.1\%) were diagnosed with breast cancer $(p=0.16)$. A majority of discordant recalls among the radiologists consisted of BI-RADS 0 recalls $(76.1 \%, 759 / 998)$.

The technologists would have recalled fewer discordant BI-RADS 0 than discordant BI-RADS 4/5 recalls (31.6\% (240/759) versus $40.6 \%$ (97/239), $p=0.01$ ). Among the 759 women with a discordant BI-RADS 0 radiologist recall, the proportion of breast cancer cases was comparable for women with or without a positive technologist assessment (5.0\% (12/240) versus $4.6 \%(24 / 519), p=0.8)$. This was also the case for discordant $\mathrm{BI}$ RADS $4 / 5$ radiologist recalls $(28.9 \%(28 / 97)$ versus $25.4 \%(36 / 142), p=0.5)$. The arbitration of discordant reading by technologists is displayed in Figure 4.1.1.

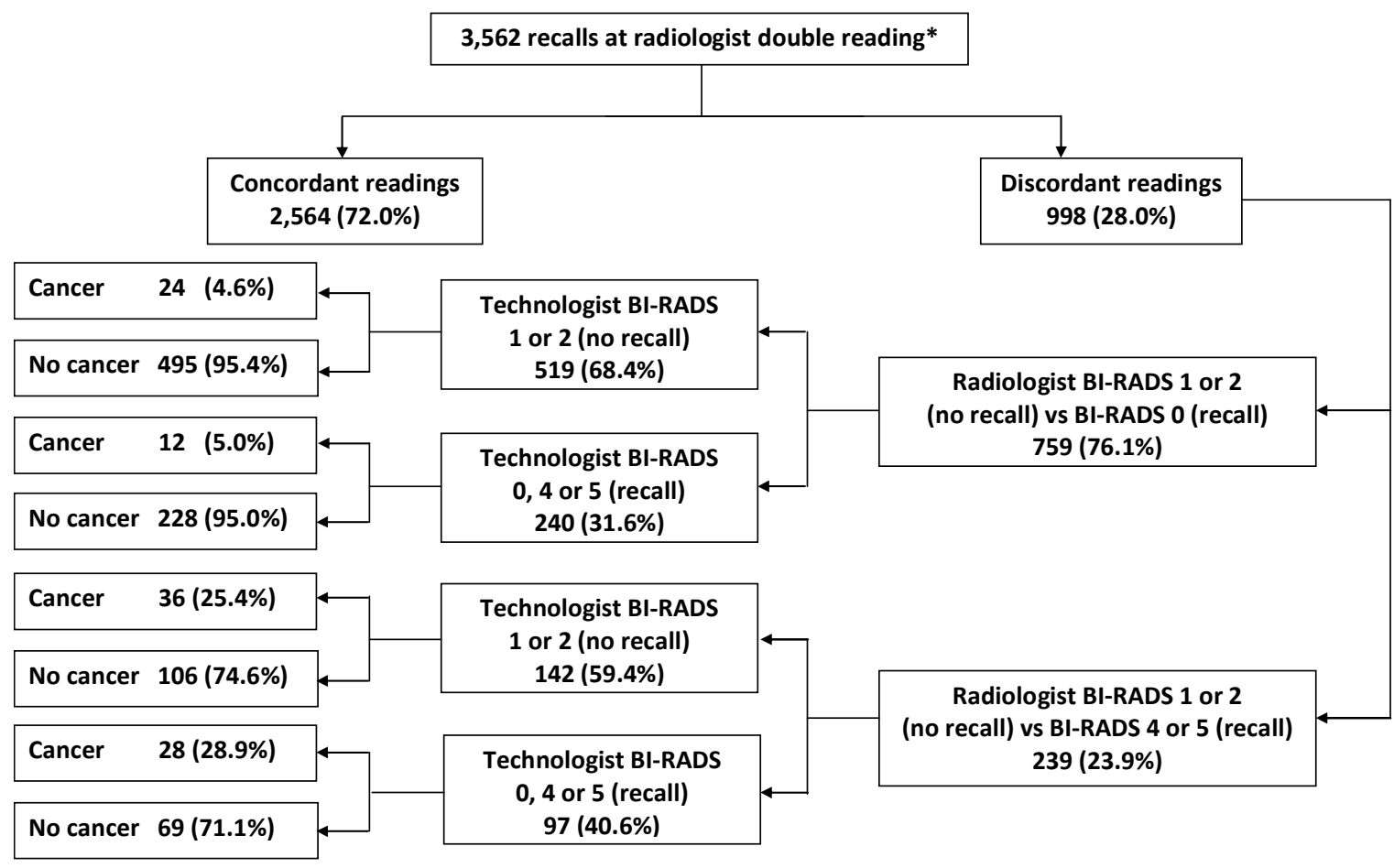

Figure 4.1.1 Technologist assessment of discordant BI-RADS readings among radiologists at screening mammography. *At radiologist double reading, a woman was recalled if the mammogram was considered to be positive by both radiologists or, in the case of discordant readings, if at least one radiologist considered recall necessary. Thus, all discordant readings were recalled, without consensus reading or arbitration being performed. 
Technologist assessments of discordant readings among screening radiologists according to mammographic abnormality

A majority of the 998 discordant readings among screening radiologists consisted of suspicious masses (71.4\%), followed by suspicious microcalcifications (11.4\%) and architectural distortions (9.3\%, Figure 4.1.2).

The technologists would have recalled a significantly larger proportion of discordant assessments for masses with microcalcifications than architectural distortions $(50.0 \%(12 / 24)$ versus $22.6 \%(21 / 93), p=0.008)$. Among women with a positive technologist assessment, the probability of breast cancer was highest in case of suspicious microcalcifications and lowest for suspicious masses $(30.4 \%(17 / 56)$ versus $7.0 \%(16 / 228), p<0.001)$. Breast cancers were diagnosed in all groups of mammographic abnormalities, except in women with a suspicious asymmetry at radiologist recall and a negative technologist assessment (Figure 4.1.2). None of these 34 recalled women proved to have breast cancer at 2-year follow-up.

Screening outcome after arbitration of discordant radiologist recalls by technologists

Table 4.1.1 shows the overall screening outcome after arbitration of all discordant radiologist recalls by technologists. The recall rate and cancer detection rate would have decreased, from 3.6 to $2.9 \%(2901 / 99,013 ; p<0.001)$ and from 7.1 to $6.5 \%$ $(644 / 99,013 ; p=0.10)$, respectively. The PPV of recall would have increased from 19.8 to $22.2 \%$ (644/2901; $p=0.02$ ). The 60 breast cancer cases that would not have been recalled by the technologists comprised $13(21.7 \%)$ cases of ductal carcinoma in situ and $47(78.3 \%)$ invasive cancers. Of the 13 DCIS cases, respectively $4(30.8 \%)$, $7(53.8 \%)$, and $2(15.4)$ were low, intermediate or high grade. Of the 47 invasive cancers, respectively $30(63.8 \%), 15(31.9 \%)$, and $2(4.3 \%)$ were stage T1a-b $(\leq 10 \mathrm{~mm})$, T1b $(11-20 \mathrm{~mm})$, and $\mathrm{T} 2+(>20 \mathrm{~mm})$ and 8 invasive cancers showed lymph node metastases. Respectively $28(59.6 \%), 13(27.7 \%)$, and $6(12.8 \%)$ of the 47 invasive cancers were Bloom and Richardson grade I, II, and III. 


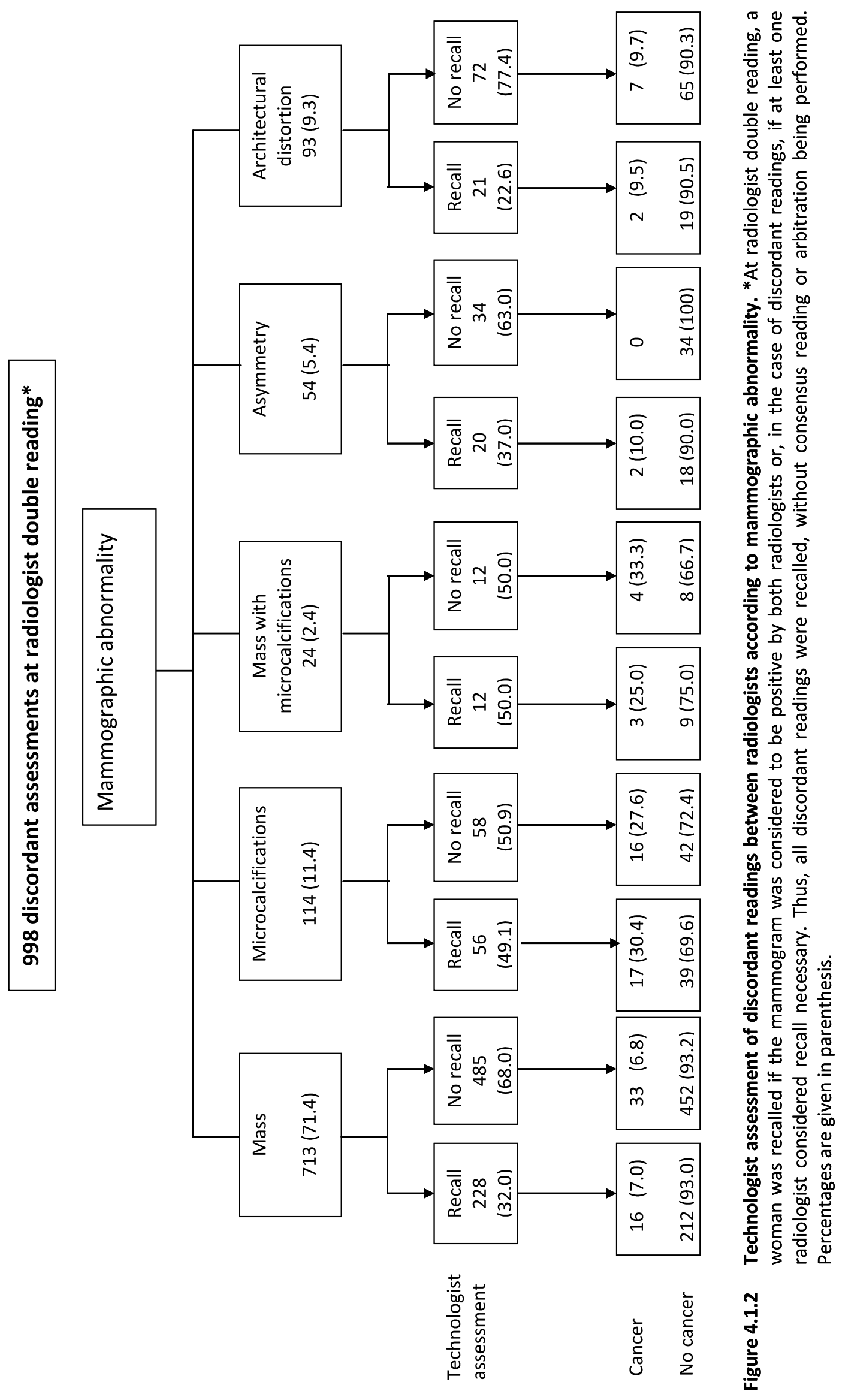


Table 4.1.1 Screening outcome without and with arbitration of discordant radiologist recalls by technologists.

\begin{tabular}{lccc}
\hline & $\begin{array}{c}\text { Outcome without } \\
\text { technologist arbitration }\end{array}$ & $\begin{array}{c}\text { Outcome after } \\
\text { technologist arbitration }\end{array}$ & P-value \\
\hline Recalls, No & 3562 & 2901 & \\
Recall rate, \% & 3.6 & 2.9 & $\mathrm{p}<0.001$ \\
Screen-detected cancers, No & 704 & 644 & $\mathrm{P}=0.10$ \\
Cancer detection rate* & 7.1 & 6.5 & $\mathrm{p}=0.02$ \\
PPV of recall, \% & 19.8 & 22.2 & $\mathrm{P}=0.94$ \\
Type of screen-detected cancer, No (\%) & & & \\
DCIS & $130(18.5)$ & $117(18.2)$ & $\mathrm{p}=0.72$ \\
Invasive cancer & $574(81.5)$ & $527(81.8)$ & \\
Tumour stage of invasive cancers, No (\%) & & & \\
T1a-b & $223(38.9)$ & $193(36.6)$ & \\
T1c & $238(41.5)$ & $223(42.3)$ & \\
T2+ & $113(19.7)$ & $111(21.1)$ & \\
\hline
\end{tabular}

* Per 1000 screens; DCIS = ductal carcinoma in situ.

\section{Discussion}

In the current study, we found that a substantial proportion of women were recalled after a discordant reading between two radiologists. The majority of discordant recalls (76.1\%) were classified as BI-RADS 0 and consisted of masses (71.4\%).

The proportion of breast cancers was comparable for discordant recalls, both $\mathrm{BI}$ RADS 0 and BI-RADS 4/5 readings, with or without positive technologist assessment. In case of a discordant reading with technologist positive assessment, the highest probability of breast cancer was found in the group with microcalcifications, and lowest in the group with suspicious masses $(p<0.001)$. Using the technologist's opinion to decide whether or not discordant readings at double reading should be recalled, lowers recall rate, and increases the PPV of recall at the cost of a lower cancer detection rate, although not statistically significant.

Discordant readings following double reading at screening mammography occur frequently. Several reading strategies have been investigated to deal with these discordant readings, though no consensus has been reached as to which strategy yields optimal results., ${ }^{3,5-7}$ In our study, we investigated the value of the technologist's assessment of discordant readings after radiologist double reading in screening mammography. Previous studies have shown that the sensitivity of technologists in reading diagnostic mammograms may be comparable to the sensitivity of radiologists, at the cost of an increased false positive recall rate. ${ }^{7,8,11-14}$ Pauli et al. showed that adding technologist reading to radiologist single reading causes a significant increase 
in sensitivity. ${ }^{11}$ Tonita et al. found that a substantial number of cancers were found with technologist review and that this was also a cost-effective alternative to radiologist double reading. ${ }^{14}$ Duijm et al. studied the effect of type and number of readers on screening outcome and concluded that highest sensitivity was obtained by quadruple interpretation with recall of all radiologist- and technologist positive readings and that triple reading by one radiologist and two technologists may be an alternative to radiologist double reading in programmes with a shortage of radiologists. ${ }^{3}$ More recently, a study by Torres-Mejia et al. showed that technologists sensitivity was comparable but their false positive rate was higher compared to the sensitivity and false positive rate of United States radiologists. ${ }^{8}$

As mentioned earlier, the proportion of breast cancer was comparable for discordant recalls with or without positive technologist assessment. This demonstrates that assessment by a technologist does not provide a significant discriminating ability in these groups. This finding contrasts with earlier studies on the added value of technologists' assessment. First, the advent of digital mammography has improved imaging quality and increased detection rate when compared to screenfilm mammography, the latter being employed in earlier reports on the added value of technologists in mammography screening. ${ }^{15-17}$ Second, a difference in reading strategies could explain the limited value of a technologists' assessment. Double reading by a second screening radiologist has been proven to increase cancer detection rate when compared to single reading, although certain studies show a limited increase in cancer detection. . $^{3,18,19}$

In the currently studied screening setting, screening radiologists review large numbers of screening mammograms, and they obtain feedback through clinical follow-up data at the hospital where they are employed and through quality improvement programmes. In case of a discordant reading between two radiologists, the limited benefit of a technologist assessment may be due to the fact that technologists do not routinely review the same large numbers of screening mammograms and receive less feedback on screening.

Although the difference in cancer detection rate was not statistically significant, 60 cancers would not have been recalled after arbitration by a technologist. These cancers comprised 9 intermediate to high-grade DCIS and 6 high-grade invasive carcinomas. Eight of these discordant readings with a negative technologist assessment were lymph node-positive malignant lesions. ${ }^{20}$ In case of a discordant reading concerning an asymmetry, using the technologist's opinion to decide whether or not to recall would not have caused any missed cancers. In all other groups of mammographic abnormalities, a negative technologist assessment would have caused missed cancers. 
To our knowledge, this is the first study to investigate the value of technologist assessment in deciding whether discordant readings among radiologists at digital screening mammography should be recalled. Other studies focus on the benefit of additional reading by the technologist in screening mammography. The major strength of our study is the large number of included screening mammograms and recalled women. Also, near complete follow-up is available on all discordant readings, allowing a careful investigation of clinical outcome and correlation with screening characteristics and the technologist's assessment of the corresponding screening mammogram.

This study also has certain limitations. First, the screening radiologists were not blinded to the technologist's assessment. Duijm et al. previously found that technologists may detect additional cancers not found by a screening radiologist. ${ }^{3}$ This could introduce bias when reviewing the screening mammogram. Statistical correction for this bias is difficult and therefore this possible bias has to be taken into account when interpreting data. Nevertheless, the goal of the current study was not to determine the value of technologist reading on general screening outcome, but to assess whether this reading might discriminate certain types of discordant readings for which recall is not indicated. Second, experience of the radiologist or technologist performing arbitration in the case of discordant readings may introduce bias. Although no reports are available, to our knowledge, a less experienced screening radiologist or technologist performing arbitration may choose to recall discordant readings more frequently than a more experienced screening radiologist or technologist.

In conclusion, our results show that additional assessment by a technologist does not provide a significant discriminating ability in case of a discordant radiologist reading. Therefore, there appears to be no added value of using the technologist assessment of screening mammograms to decide whether or not discordant radiologist readings should be recalled. This strategy lowers the recall rate but at the expense of a, borderline significant, decrease in cancer detection rate. 


\section{References}

1. Sankatsing VDV, van Ravesteyn NT, Heijnsdijk EAM, et al. The effect of population-based mammography screening in Dutch municipalities on breast cancer mortality: 20 years of follow-up. Int J Cancer 2017;141:671-677.

2. European Commission. Directorate-general for health and consumer protection, Perry N, Puthaar E. European guidelines for quality assurance in breast cancer screening and diagnosis, 4 th edn. Office for Official Publications of the European Communities, Luxembourg 2006.

3. Duijm LE, Louwman MW, Groenewoud JH, van de Poll-Franse LV, Fracheboud J, Coebergh JW. Interobserver variability in mammography screening and effect of type and number of readers on screening outcome. Br J Cancer 2009;100:901-907.

4. Gur D, Sumkin JH, Hardesty LA, et al. Recall and detection rates in screening mammography. Cancer 2004;100:1590-1594.

5. Klompenhouwer EG, Voogd AC, den Heeten GJ, et al. Discrepant screening mammography assessments at blinded and nonblinded double reading: impact of arbitration by a third reader on screening outcome. Eur Radiol 2015;25:2821-2829.

6. Shaw CM, Flanagan FL, Fenlon HM, McNicholas MM. Consensus review of discordant findings maximizes cancer detection rate in double-reader screening mammography: Irish National Breast Screening Program experience. Radiology 2009;250:354-362.

7. Duijm LE, Groenewoud JH, Fracheboud J, de Koning HJ. Additional double reading of screening mammograms by radiologic technologists: impact on screening performance parameters. J Natl Cancer Inst 2007;99:1162-1170.

8. Torres-Mejia G, Smith RA, Carranza-Flores Mde L, et al. Radiographers supporting radiologists in the interpretation of screening mammography: a viable strategy to meet the shortage in the number of radiologists. BMC Cancer 2015;15:410.

9. Klompenhouwer EG, Voogd AC, den Heeten GJ, et al. Blinded double reading yields a higher programme sensitivity than non-blinded double reading at digital screening mammography: a prospected population based study in the south of The Netherlands. Eur J Cancer 2015;51:391-399.

10. American College of Radiology. BI-RADS Committee. ACR BI-RADS atlas: breast imaging reporting and data system, 5th edn. American College of Radiology, Reston 2013.

11. Pauli R, Hammond S, Cooke J, Ansell J. Comparison of radiographer/radiologist double film reading with single reading in breast cancer screening. J Med Screen 1996;3:18-22.

12. van den Biggelaar FJ, Nelemans PJ, Flobbe K. Performance of radiographers in mammogram interpretation: a systematic review. Breast 2008;17:85-90.

13. Debono JC, Poulos AE, Houssami N, Turner RM, Boyages J. Evaluation of radiographers' mammography screen-reading accuracy in Australia. J Med Radiat Sci 2015;62:15-22.

14. Tonita JM, Hillis JP, Lim CH. Medical radiologic technologist review: effects on a population-based breast cancer screening program. Radiology 1999;211:529-533.

15. Pisano ED, Gatsonis C, Hendrick E, et al. Diagnostic performance of digital versus film mammography for breast-cancer screening. N Engl J Med 2005;353:1773-1783.

16. Bluekens AM, Holland R, Karssemeijer N, Broeders MJ, den Heeten GJ. Comparison of digital screening mammography and screen-film mammography in the early detection of clinically relevant cancers: a multicenter study. Radiology 2012;265:707-714.

17. van Luijt PA, Fracheboud J, Heijnsdijk EA, den Heeten GJ, de Koning HJ, National Evaluation Team for Breast Cancer Screening in Netherlands Study G. Nation-wide data on screening performance during the transition to digital mammography: observations in 6 million screens. Eur J Cancer 2013;49:35173525.

18. Posso M, Puig T, Carles M, Rue M, Canelo-Aybar C, Bonfill X. Effectiveness and cost-effectiveness of double reading in digital mammography screening: A systematic review and metaanalysis. Eur J Radiol 2017;96:40-49. 
19. Klompenhouwer EG, Weber RJ, Voogd AC, et al. Arbitration of discrepant BI-RADS 0 recalls by a third reader at screening mammography lowers recall rate but not the cancer detection rate and sensitivity at blinded and non-blinded double reading. Breast 2015;24:601-607.

20. Otten JD, Karssemeijer N, Hendriks JH, et al. Effect of recall rate on earlier screen detection of breast cancers based on the Dutch performance indicators. J Natl Cancer Inst 2005;97:748-754. 



\section{Chapter 4.2}

Additional breast cancer detection at digital screening mammography through quality assurance sessions between technologists and radiologists 


\section{Abstract}

\section{Background}

Screening technologists may function as readers in breast cancer screening programmes. In the Netherlands, they attend quality assurance sessions. The frequency and characteristics of additional breast cancers detected through these sessions have not been reported.

\section{Purpose}

To determine the frequency and characteristics of cancers detected through quality assurance sessions.

\section{Materials and methods}

This secondary analysis of a prospective cohort included 466,647 screening mammograms obtained between January 1, 2009, and January 1, 2017. Mammograms were single read by certified screening technologists before being double read by two certified screening radiologists who were not blinded to the technologist's reading. The technologists and a coordinating screening radiologist regularly discussed mammograms that the technologists considered suspicious but that did not prompt recall at radiologist double reading. The coordinating radiologist decided whether secondary recall was indicated. During a 2-year follow-up, radiologic and pathologic outcome data for all recalled women were obtained. Characteristics of cancers detected at radiologist double reading and those detected through quality assurance sessions were compared by using $\chi^{2}$ and Fisher exact tests.

\section{Results}

A total of 14,142 women (mean age, 59 years \pm 7.8 [standard deviation]; range, 49-75 years) were recalled (recall rate, 3.0\% [14 142 of 466 647]): 14057 after radiologist double reading and 85 by the coordinating radiologists after quality assurance sessions. This resulted in 3156 screen-detected cancers (6.8 cancers detected per 1000 screens), of which 26 ( $0.8 \%$ of screendetected cancers [26 of 3156]) were detected after secondary recall through quality assurance sessions. The latter comprised eight ductal carcinomas in situ ( $88 \%$ intermediate or high grade [seven of eight]) and 18 invasive cancers (14 T1a-c and four T2+ cancers, 89\% Nottingham grade I or II [16 of 18]). No significant differences in tumour characteristics were found ( $p$ values ranging from 0.22 to 0.95 ). Sensitivity of quality assurance sessions for additional cancer detection was $52 \%$ ( 26 of 50 ; $95 \%$ confidence interval: $38 \%, 66 \%$ ).

\section{Conclusion}

The role of quality assurance sessions in additional cancer detection is limited. Tumour characteristics did not differ significantly from those of cancers detected at radiologist double reading. 


\section{Introduction}

Breast cancer is the most common cancer diagnosed in women worldwide, with an incidence of more than 2 million in 2018. ${ }^{1}$ Every year, more than 14,500 invasive breast cancers and more than 2300 in situ breast cancers are diagnosed in women in the Netherlands. In 2017, 3132 women died of breast cancer in the Netherlands. ${ }^{2}$ Since the gradual implementation of the Dutch nationwide breast cancer screening programme from 1987 through 1997, breast cancer mortality has declined by more than $30 \%$ in women aged $55-79$ years. ${ }^{3}$ This mortality reduction is most likely a result of earlier cancer detection and substantial improvements in breast cancer treatment.

Previous studies, mostly dating from the screen-film mammography era, have investigated the role of technologists in reading screening mammograms. These studies have shown that trained screening mammography technologists may identify breast cancers that are missed by screening radiologists ${ }^{4,5}$ and that double reading by a radiologist and a technologist could be an alternative to radiologist double reading in programmes with a shortage of radiologists. ${ }^{6-8}$ In a recent study ${ }^{9}$, we found no added value of using the technologist assessment of digital screening mammograms to decide whether discordant radiologist readings at blinded double reading should be recalled.

Since the initiation of breast cancer screening in our screening region, technologists have been encouraged to look for mammographic abnormalities. After obtaining a mammogram, technologists classify it according to the Breast Imaging Reporting and Data System (BI-RADS). All mammograms are then double read by a team of certified screening radiologists. At regular 6-week intervals, the screening technologists discuss with a coordinating screening radiologist the mammograms of women who are not recalled by the screening radiologists but whose mammograms are considered suspicious by the technologists. The coordinating radiologist then decides for each case whether secondary recall is indicated.

The purpose of our study was to determine the frequency and characteristics of cancers additionally detected through quality assurance sessions, as well as those of screen-detected cancers and interval cancers with a technologist-positive screening in a previous screening round. 


\section{Materials and methods}

\section{Study population}

This retrospective study using a prospectively obtained database was performed in the Dutch nationwide breast cancer screening programme, which offers biennial screening mammography to asymptomatic women aged $50-75$ years. We included all 466,647 women who underwent screening mammography at one of four specialized screening units (Eindhoven, De Kempen, Den Bosch, and De Meierij) in a southern screening region (Bevolkingsonderzoek Zuid) between January 1, 2009, and January 1, 2017. In our screening region, transition from screen-film mammography to full-field digital mammography took place between 2009 and 2010. A small portion of included mammograms, consisting of both initial and subsequent screens, were therefore screen-film mammograms. On entering the screening programme, all women are routinely asked to give written informed consent to use their data for quality assurance and scientific purposes. Two women did not give this permission and were therefore excluded from our analysis. According to the Dutch Central Committee on Research involving Human Subjects, our study did not require ethical approval. Institutional review board approval was also waived.

\section{Screening procedure, readers, and recall}

Details of the Dutch nationwide breast cancer screening programme have been described previously. ${ }^{10,11}$ In brief, all two-view mammograms were obtained by a team of specialized screening mammography technologists. Screen-film mammograms were obtained with commercially available units (Performa; Oldelft, Tuusula, Finland), and full-field digital mammograms were acquired with a Lorad Selenia system (Hologic, Danbury, Conn) with a $70-\mu \mathrm{m}$ pixel size and a $232 \times 286-\mathrm{mm}$ field of view. Each mammogram was single read at the time of acquisition by the technologist who obtained it, before being double read by two certified screening radiologists, who were not blinded to the technologist's reading. The radiologists' reading took place 2 or 3 days after the mammogram was obtained. Mammograms are classified according to the BI-RADS classification, ${ }^{12}$ which was implemented in our screening programme in 2008 and has been used routinely and mentioned in recall reports since 2009 .

Technologists working at screening units receive extensive training before their employment. Until 2015, this training was provided by the Dutch Reference Centre for Screening. Since 2015, the regional screening organisations provide the training for their technologists themselves. Instruction on mammography technique, positioning, and evaluation of the images for technical quality is central to this training. In 
addition, technologists are also trained in breast anatomy and mammographic features of benign and malignant breast disease. All technologists receive further training at the Dutch Reference Centre for Screening yearly and are required to attend mammography conferences and symposia every 1-2 years. A total of 42 technologists were employed at our four screening units during our study period; 24 of them were employed from the start of the study, and 18 joined later. Two technologists quit in 2014. The mean duration of experience of screening technologists at the start of the study was 116 months (range, 4-208 months).

Every 6 weeks, the technologists attend a quality assurance session where a coordinating screening radiologist discusses all the recall decisions with the technologists, as well as all false negative cases (i.e., interval cancers). This coordinating radiologist is one of the 13 certified screening radiologists, thus also functioning as a reader in the screening programme. Mammograms of all women who were not recalled by the radiologists but had a technologist-positive screening were also discussed at these sessions. At this stage, a woman was recalled if the coordinating radiologist considered work-up necessary.

As described earlier, each mammogram was read by one of the technologists at the time of acquisition, using a technologist workstation with a 3-MP monitor in a room where the lights were not routinely dimmed. Viewing conditions for technologists were not controlled during this study. The technologists classified the mammogram according to BI-RADS. ${ }^{12}$ Like the radiologists, they were allowed to use all BI-RADS categories except BI-RADS category 3. The technologist's classification was visible only to the screening radiologists and should be seen as a way of conveying to the radiologists their level of suspicion in case of an abnormality. In case of a subsequent screening, the previous screening examination was available for comparison. In case of a positive screening (i.e., BI-RADS category 0,4 , or 5), the technologist classified the abnormality according to one of six categories: suspicious mass, suspicious microcalcifications, mass with microcalcifications, architectural distortion, asymmetry, or other abnormality.

Two to 3 days later, all mammograms were double read by a team of 13 certified screening radiologists (including one of the authors, L.E.M.D., a radiologist with 13 years of experience as a screening radiologist at the start of our study). Ten of them already worked at the screening programme before the start of our study, and three joined later (in 2010, 2011, and 2012, respectively). At the start of our study, mean duration of experience of these radiologists in reading screening mammograms was 136 months (range, 15-167 months). The radiologists were not blinded to the technologist's interpretation. Radiologists performed their readings on a 5-MP workstation in a room devoid of daylight. In case of a subsequent screening, all 
previous screening examinations were available for comparison. Both radiologists classified the mammogram according to BI-RADS, and, in case of a mammographic abnormality, according to one of the six categories mentioned earlier. Women with BI-RADS category 1 or 2 results were not recalled, whereas women with BI-RADS category 0,4 , or 5 results were referred for additional work-up at the breast unit of a nearby hospital. BI-RADS category 3 is not used as a classification for screening examinations because short-term follow-up is not available within the screening programme itself. BI-RADS category 3 is used in clinical work-up after recall. In case of a discordant reading between the two radiologists, where one classified the mammogram as BI-RADS category 0,4 , or 5 (i.e., positive, prompting recall) and the other as BI-RADS category 1 or 2 (i.e., negative, not prompting recall), the woman in question was recalled without consensus reading between the two radiologists. From 2016 on, discordant readings underwent arbitration by a third radiologist.

\section{Diagnostic work-up and follow-up after recall}

After physical examination of a recalled woman by a surgical oncologist or dedicated breast nurse, additional mammographic and/or tomosynthetic views were obtained if necessary. Previous screening mammograms were routinely available for comparison through the hospital's picture archiving and communication system. The clinical radiologists classified imaging findings according to BI-RADS. BI-RADS category 4 and 5 lesions were routinely biopsied, and BI-RADS category 3 lesions were biopsied or followed up. Depending on clinical and imaging findings, further work-up could consist of one or more of the following: breast US, MRI, percutaneous fine-needle aspiration, core-needle biopsy, stereotactic biopsy, or open surgical biopsy. During a follow-up period of approximately 2 years (until the next biennial screening examination), all clinical, imaging, and pathologic data were collected for all recalled women. All data were stored by L.E.M.D. in a computerized spreadsheet (Excel; Microsoft, Redmond, Wash). In case of a cancer detected after a subsequent screening examination, one of the radiologists (L.E.M.D.) checked the screening information system to see if there was a technologist-positive screening in a previous screening round. If this was the case, two radiologists reviewed the mammograms and determined in consensus whether the abnormalities were the same.

\section{Detection and review of interval cancers}

Interval cancers were defined as breast cancers diagnosed in women after a previous negative screening mammogram and before subsequent screening mammography was performed. A validated connection between the Dutch National Comprehensive Cancer Centre and the screening information system was available for the screening period 2009-2014. For the screening period 2015-2016, this connection was not 
available. Methods for tracing interval cancers for the latter period have been described in detail previously. ${ }^{13}$ For all interval cancers, the screening information system was also checked for a technologist-positive screening in a previous round. If this was the case, again, two radiologists reviewed the images and determined in consensus whether the abnormalities were the same.

\section{Statistical analysis}

Primary outcome measures were recall rate (recalls per 100 screens), cancer detection rate (screen-detected cancers per 1000 screens), interval cancer rate (interval cancers per 1000 screens), false-positive recall rate (false-positive recalls per 1000 screens), and positive predictive value of recall. We performed $\chi^{2}$ tests and Fisher exact tests to test differences in mammographic characteristics and an array of tumour characteristics between quality assurance recalls, radiologist recalls (those with and those without a previous positive screening by a technologist), and interval cancers. In case of bilateral disease, the most advanced tumour was included in our analysis. In case of multicentric or multifocal breast cancer, only the largest tumour was included in the analysis. $p<0.05$ indicated a statistically significant difference. Statistical analysis was performed by one author (A.M.P.C.) using SPSS Statistics 23.0 (IBM, Armonk, NY).

\section{Results}

\section{Overall screening results}

Of 466,647 screened women, 14,142 were referred for further diagnostic assessment of a mammographic abnormality (recall rate, 3.0\%). Breast cancer was diagnosed in 3156 women (Figure 4.2.1), resulting in a cancer detection rate of 6.8 per 1000 screens and a false-positive rate of 23.5 per 1000 screens. The positive predictive value of recall, irrespective of recall BI-RADS classification, was $22 \%$. Programme sensitivity was $82 \%$ (3156 of 3867). Technologists would have recalled 2320 (including 26 through quality assurance sessions) of 3156 women with a screendetected cancer, resulting in a cancer detection rate of 5.0 per 1000 screens. This means that 830 cancers (26\% [ 830 of 3156 ]) that were detected by the radiologists would have been missed by the technologists.

\section{Recall after quality assurance sessions}

During our inclusion period of 8 years, technologists would have recalled 11,627 women (9783 [84\%] with BI-RADS category 0 results, 1817 [16\%] with BI-RADS 
category 4 results, and 27 [0.2\%] with BI-RADS category 5 results) whom the radiologists classified as having BI-RADS category 1 or 2 results (i.e., negative, with no recall). All of these mammograms were discussed at quality assurance sessions. Of these, 85 (0.7\%) were recalled after these quality assurance sessions (Figure 4.2.1). Breast cancer was diagnosed in 26 of 85 women, resulting in a $31 \%$ positive predictive value of a quality assurance recall. In other words, $0.2 \%$ (26 of 11627 ) of women who were not recalled at radiologist double reading but had a mammogram considered suspicious by the technologist were in fact diagnosed with breast cancer. The sensitivity of quality assurance recalls for additional cancer detection was 52\% (26 of 50; $95 \%$ confidence interval: 38\%, 66\%). Compared with the 3130 screening-detected cancers diagnosed after a standard radiologist recall, there were no significant differences in tumour characteristics (Table 4.2.1), with $p$ values ranging from 0.23 to 0.95. There were also no significant differences in mammographic characteristics: screen-film mammography versus full-field digital mammography $(p=0.11)$, type of mammographic lesion ( $p=0.56)$, and tissue density $(p=0.28)$.

\section{Technologist-positive screen in a previous screening round}

Of 3156 women with a screen-detected cancer, 104 had a positive technologist screen for the same abnormality in a previous screening round (Figures 4.2.2, 4.2.3) and were, at that time, not recalled at radiologist double reading or after quality assurance sessions. After 2 years of follow-up, interval cancers had been diagnosed in 711 women, yielding an interval cancer rate of 1.5 per 1000 screens. There were 24 interval cancers among the 11542 women who were not recalled after quality assurance sessions (0.2\% [24 of 11 542]) and 687 interval cancers among the 440963 women with a negative screening by both technologists and radiologists $(0.2 \%$ [687 of 440 963]) (Figure 4.2.1). There were no interval cancers in the women who experienced a secondary recall after quality assurance sessions. 


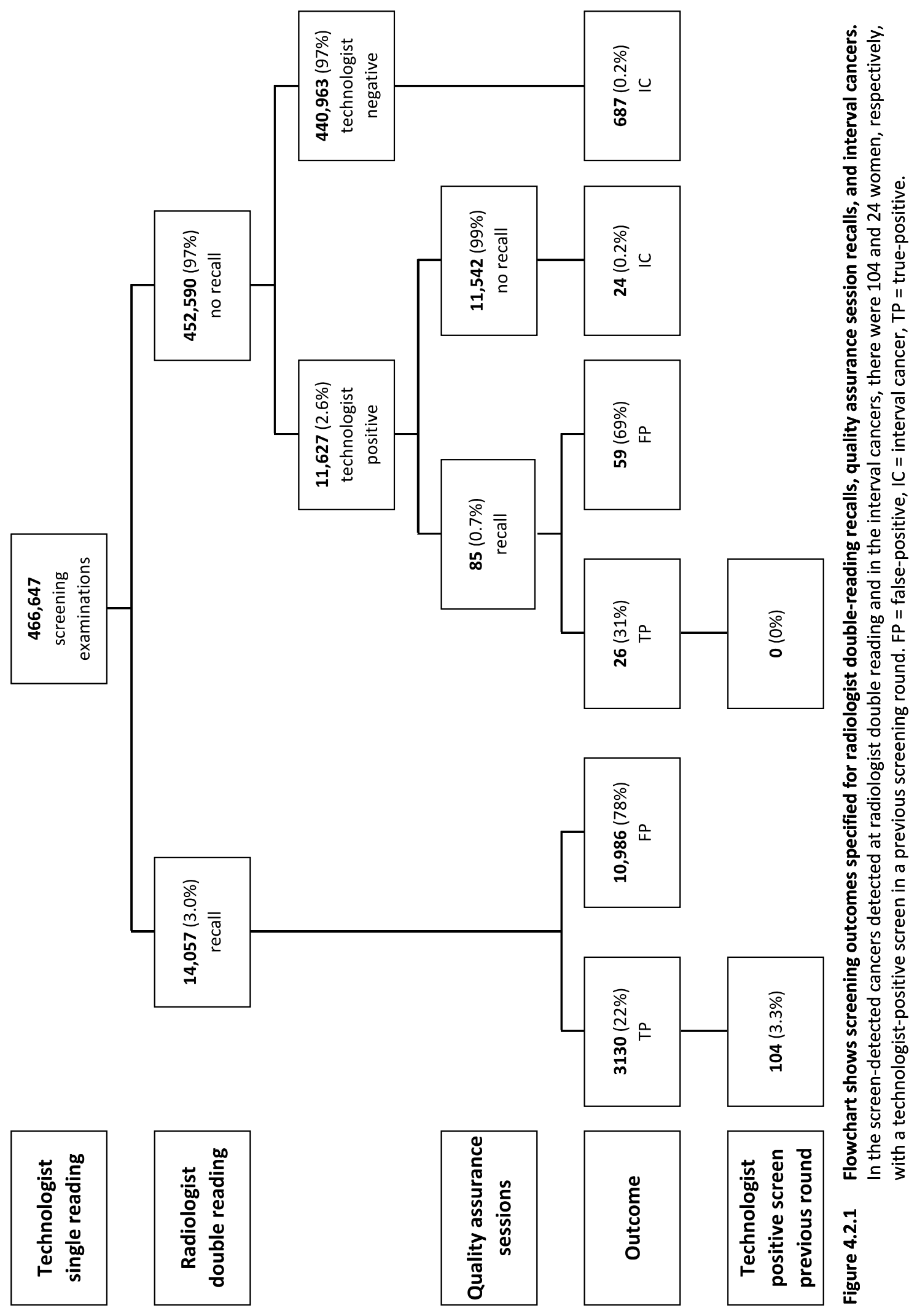


Table 4.2.1 Standard (radiologist double reading) recalls versus quality assurance recalls: tumour characteristics of screening detected cancers.

\begin{tabular}{|c|c|c|c|c|c|}
\hline $\begin{array}{l}\text { Tumour characteristic } \\
\text { Type of cancer, No (\%) }\end{array}$ & \multicolumn{2}{|c|}{$\begin{array}{c}\text { Standard recall } \\
(n=3130)\end{array}$} & \multicolumn{2}{|c|}{$\begin{array}{l}\text { Quality assurance } \\
\text { recall }(n=26)\end{array}$} & $\begin{array}{r}\text { P-value } \\
0.225\end{array}$ \\
\hline DCIS & 651 & $(20.8)$ & 8 & $(30.8)$ & \\
\hline Invasive & 2479 & (79.2) & 18 & (69.2) & \\
\hline DCIS grade, No (\%) & & & & & 0.670 \\
\hline Low & 132 & $(20.3)$ & 1 & $(12.5)$ & \\
\hline Intermediate & 230 & $(35.3)$ & 4 & $(50.0)$ & \\
\hline High & 289 & $(44.4)$ & 3 & $(37.5)$ & \\
\hline Histology of invasive cancers, No (\%) & & & & & 0.809 \\
\hline Ductal & 1951 & (79.0) & 14 & $(77.8)$ & \\
\hline Lobular & 293 & (11.9) & 3 & $(16.7)$ & \\
\hline Mixed ductal/lobular & 85 & $(3.4)$ & 1 & $(5.6)$ & \\
\hline Other & 139 & $(5.5)$ & 0 & $(0.0)$ & \\
\hline Unknown & 1 & $(0.0)$ & 0 & $(0.0)$ & \\
\hline Tumour size of invasive cancers, No (\%) & & & & & 0.935 \\
\hline $\mathrm{T} 1(\leq 20 \mathrm{~mm})$ & 1978 & $(79.8)$ & 14 & $(77.8)$ & \\
\hline $\mathrm{T} 2+(>20 \mathrm{~mm})$ & 491 & $(19.8)$ & 4 & $(22.2)$ & \\
\hline Unknown (Tx) & 10 & $(0.4)$ & 0 & $(0.0)$ & \\
\hline Lymph node status of invasive cancers, No (\%) & & & & & 0.411 \\
\hline $\mathrm{N}+$ & 535 & $(21.6)$ & 6 & $(33.3)$ & \\
\hline $\mathrm{N}-$ & 1884 & $(76.0)$ & 12 & $(66.7)$ & \\
\hline Unknown (Nx) & 60 & $(2.4)$ & 0 & $(0.0)$ & \\
\hline Nottingham grade, No (\%) & & & & & 0.947 \\
\hline 1 & 1116 & $(45.0)$ & 9 & $(50.0)$ & \\
\hline II & 1063 & (42.9) & 7 & (38.9) & \\
\hline III & 272 & $(11.0)$ & 2 & $(11.1)$ & \\
\hline Unknown & 28 & $(1.1)$ & 0 & $(0.0)$ & \\
\hline Oestrogen receptor status, No (\%) & & & & & 0.528 \\
\hline Positive & 2240 & $(90.4)$ & 15 & $(83.3)$ & \\
\hline Negative & 227 & $(9.2)$ & 3 & $(16.7)$ & \\
\hline Unknown & 12 & $(0.5)$ & 0 & $(0.0)$ & \\
\hline Progesterone receptor status, No (\%) & & & & & 0.822 \\
\hline Positive & 1788 & $(72.1)$ & 12 & $(66.7)$ & \\
\hline Negative & 679 & $(27.4)$ & 6 & (33.3) & \\
\hline Unknown & 12 & $(0.5)$ & 0 & $(0.0)$ & \\
\hline Her2/Neu receptor status, No (\%) & & & & & 0.784 \\
\hline Positive & 233 & $(9.4)$ & 1 & $(5.6)$ & \\
\hline Negative & 2224 & (89.7) & 17 & (94.4) & \\
\hline Unknown & 22 & $(0.9)$ & 0 & $(0.0)$ & \\
\hline Triple negative tumour, No (\%) & & & & & 0.553 \\
\hline Yes & 152 & $(6.1)$ & 3 & $(16.7)$ & \\
\hline No & 2327 & $(93.9)$ & 15 & $(83.3)$ & \\
\hline
\end{tabular}

DCIS = ductal carcinoma in situ, Her2/neu receptor = human epidermal growth factor receptor $2, \mathrm{Nx}=$ unknown lymph node status, $T x=$ unknown tumour size. 

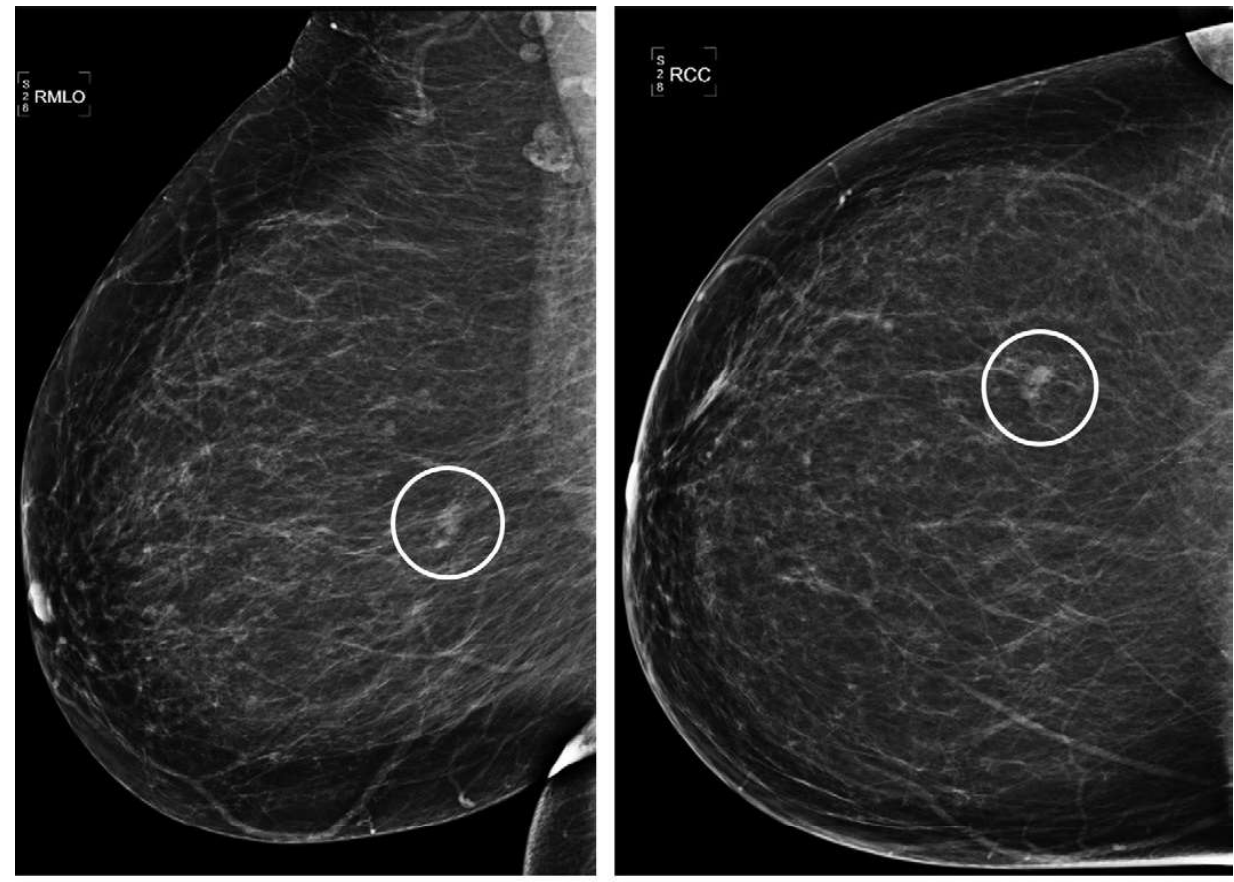

a.

b.
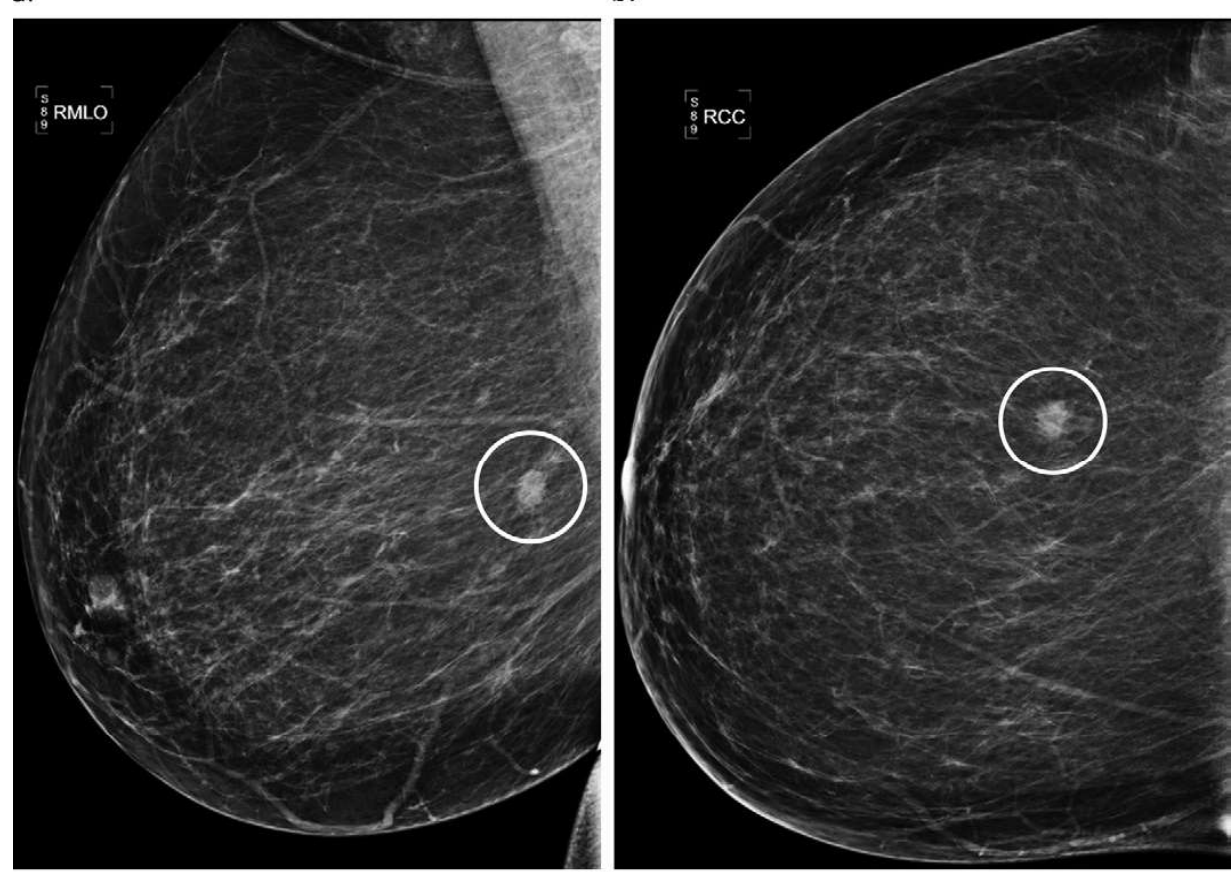

c.

d.

Figure 4.2.2 (a) Right mediolateral oblique (RMLO) view and (b) right craniocaudal (RCC) view in 54-yearold woman who underwent four-view screening mammography in 2010. This examination shows a small, well-defined mass (circle), classified by the technologist as Breast Imaging Reporting and Data System category 0 . The woman was not recalled after radiologist double reading or the quality assurance session. (c,d) Subsequent screening mammograms obtained in 2012 (in [c] RMLO view and [d] RCC view) show an increase in the size and density of the mass, which now has spiculated margins (circle). Assessment after referral revealed an 11-mm triple-negative invasive ductal carcinoma with micro metastasis in one axillary lymph node $(\mathrm{T} 1 \mathrm{cN}+)$. 


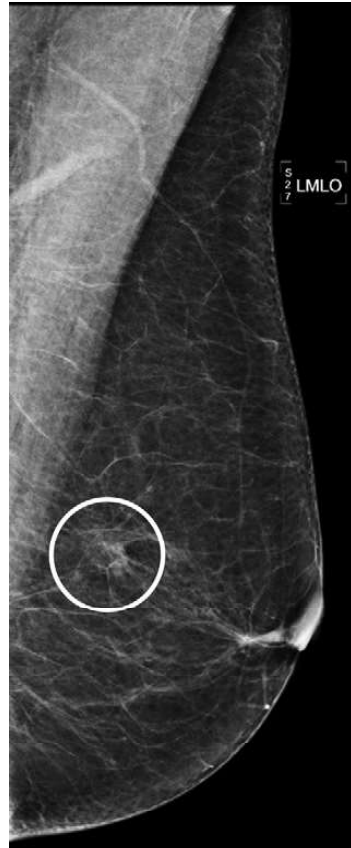

a.

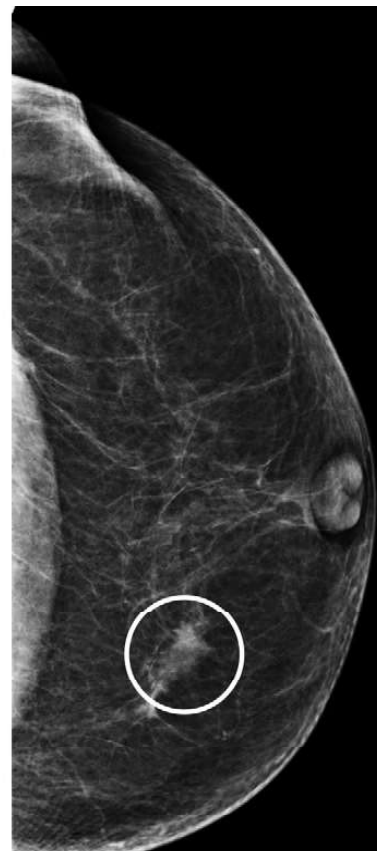

b.

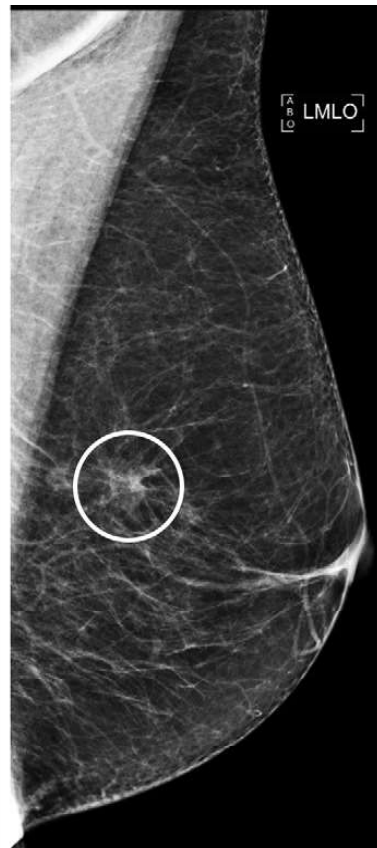

c.

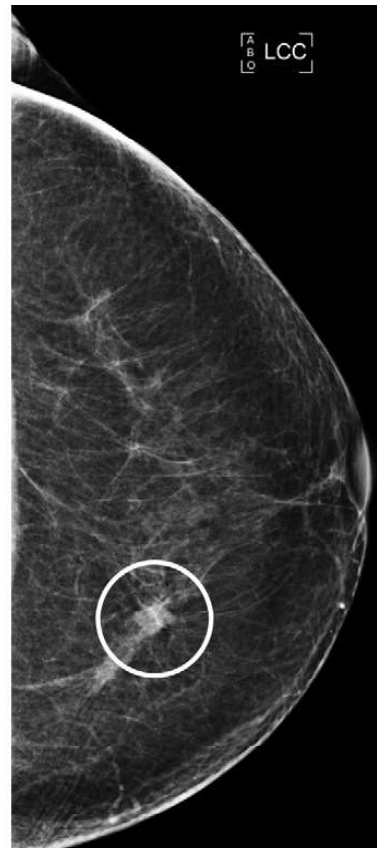

d.

Figure 4.2.3 (a) Left mediolateral oblique (LMLO) view and (b) left craniocaudal (LCC) view in 65-year-old woman who underwent four-view screening mammography in 2011. This examination shows an area of architectural distortion (circle) classified by the technologist as Breast Imaging Reporting and Data System category 4. Screening result was negative after radiologist double reading and quality assurance session. At subsequent screening mammography in 2013 (in [c] LMLO view and [d] LCC view), the area of architectural distortion was recalled. Assessment after referral revealed a $15-\mathrm{mm}$ invasive ductal carcinoma without axillary metastasis (T1cN-).

Because these 104 screen-detected cancers and 24 interval cancers could be considered as missed at quality assurance sessions at the time of the technologistpositive screen, we compared them with the 26 cancers that were detected through quality assurance sessions. We found no significant differences in mammographic characteristics: screen-film mammography versus full-field digital mammography $(p=0.40)$, type of mammographic lesion $(p=0.09)$, and tissue density $(p=0.50)$. We also found no significant differences in tumour characteristics, with $p$ values ranging from 0.10 to 0.99 (Table 4.2.2). The proportion of microcalcifications was higher in the cancers detected through quality assurance sessions (35\% [9 of 26] vs. 16\% [21 of 128], $p=0.09$ ). There was a trend toward more invasive cancers ( $84 \%$ [107 of 128] vs. $69 \%$ [18 of 26]) versus ductal carcinoma in situ (16\% [21 of 128 ] vs. $31 \%$ [8 of 26]) in the interval cancers and the screen-detected cancers with a previous technologist positive screen $(p=0.10)$. In addition, there appeared to be a trend toward more triplenegative tumours ( $17 \%$ [three of 18 ] vs. $5.6 \%$ [6 of 107], $p=0.12$ ) in the cancers detected through quality assurance recall (Table 4.2.2). 
Table 4.2.2 Quality assurance recalls versus interval cancers and SDC's with technologist positive screen in previous screening round: tumour characteristics of screen-detected cancers.

\begin{tabular}{|c|c|c|c|c|c|}
\hline $\begin{array}{l}\text { Tumour characteristic } \\
\text { Type of cancer, No (\%) }\end{array}$ & \multicolumn{2}{|c|}{$\begin{array}{l}\text { Quality assurance } \\
\text { recall }(n=26)\end{array}$} & \multicolumn{2}{|c|}{$\begin{array}{l}\text { IC's and SDC's with } \\
\text { previously positive } \\
\text { screen ( } n=128)\end{array}$} & \begin{tabular}{|c|} 
P-value \\
0.102 \\
\end{tabular} \\
\hline DCIS & 8 & $(30.8)$ & 21 & $(16.4)$ & \\
\hline Invasive & 18 & (69.2) & 107 & (83.6) & \\
\hline DCIS grade, No (\%) & & & & & 0.773 \\
\hline Low & 1 & $(12.5)$ & 5 & $(23.8)$ & \\
\hline Intermediate & 4 & $(50)$ & 10 & (47.6) & \\
\hline High & 3 & (37.5) & 6 & $(28.6)$ & \\
\hline Histology of invasive cancers, No (\%) & & & & & 0.665 \\
\hline Ductal & 14 & $(77.8)$ & 79 & $(73.8)$ & \\
\hline Lobular & 3 & (16.7) & 18 & (16.8) & \\
\hline Mixed ductal/lobular & 1 & $(5.6)$ & 3 & $(2.8)$ & \\
\hline Other & 0 & $(0.0)$ & 7 & (6.5) & \\
\hline Tumour size of invasive cancers, No (\%) & & & & & 0.273 \\
\hline $\mathrm{T} 1(\leq 20 \mathrm{~mm})$ & 14 & $(77.8)$ & 62 & (57.9) & \\
\hline $\mathrm{T} 2+(>20 \mathrm{~mm})$ & 4 & $(22.2)$ & 44 & (41.1) & \\
\hline Unknown (Tx) & 0 & $(0.0)$ & 1 & $(0.9)$ & \\
\hline Lymph node status of invasive cancers, No (\%) & & & & & 0.553 \\
\hline $\mathrm{N}+$ & 6 & $(33.3)$ & 38 & $(35.5)$ & \\
\hline $\mathrm{N}-$ & 12 & (66.7) & 63 & (58.9) & \\
\hline Unknown (Nx) & 60 & $(0.0)$ & 6 & $(5.6)$ & \\
\hline Nottingham grade, No (\%) & & & & & 0.825 \\
\hline 1 & 9 & $(50.0)$ & 43 & $(40.2)$ & \\
\hline II & 7 & (38.9) & 50 & (46.7) & \\
\hline III & 2 & (11.1) & 12 & $(11.2)$ & \\
\hline Unknown & 0 & $(0.0)$ & 2 & $(1.9)$ & \\
\hline Oestrogen receptor status, No (\%) & & & & & 0.701 \\
\hline Positive & 15 & $(83.3)$ & 94 & (87.9) & \\
\hline Negative & 3 & (16.7) & 13 & (12.1) & \\
\hline Progesterone receptor status, No (\%) & & & & & 1.000 \\
\hline Positive & 12 & $(66.7)$ & 68 & $(63.6)$ & \\
\hline Negative & 6 & (33.3) & 39 & (36.4) & \\
\hline Her2/Neu receptor status, No (\%) & & & & & 0.464 \\
\hline Positive & 1 & $(5.6)$ & 15 & $(14.0)$ & \\
\hline Negative & 17 & (94.4) & 92 & (86.0) & \\
\hline Triple negative tumour, No (\%) & & & & & 0.121 \\
\hline Yes & 3 & $(16.7)$ & 6 & $(5.6)$ & \\
\hline No & 15 & (83.3) & 101 & (94.4) & \\
\hline
\end{tabular}

DCIS = ductal carcinoma in situ, HER2/neu receptor = human epidermal growth factor receptor $2, I C=$ interval cancer, $N x=$ unknown lymph node status, $S D C=$ screening-detected cancer, $T x=$ unknown tumour size. 


\section{Discussion}

We determined the frequency and characteristics of cancers that were additionally detected through quality assurance sessions between screening technologists and coordinating screening radiologists. We found that sensitivity of quality assurance sessions for additional cancer detection was $52 \%$ and that $0.8 \%$ of screen-detected cancers were detected through quality assurance sessions. Most of these cancers were invasive and larger than $10 \mathrm{~mm}$ and therefore probably do not reflect overdiagnosis. We found no significant differences in tissue density and type of mammographic lesion. There were also no significant differences in tumour characteristics. However, we noted a trend toward more invasive cancers versus ductal carcinoma in situ in the interval cancers and screen-detected cancers with a previous technologist-positive screen and a trend toward more triple-negative tumours in the cancers detected through quality assurance sessions. The percentage of interval cancers was similar in the women who were not recalled through quality assurance sessions and those with a negative screen by both radiologists and technologists.

Several studies have explored the role of technologists as readers in breast cancer screening. Pauli et al. ${ }^{7}$ found that double reading by a radiologist and a technologist resulted in increases in sensitivity similar to those previously reported in radiologist double-reading studies. Tonita et al. ${ }^{8}$ added technologist review to single-radiologist reading to select cases for blind reading by a second radiologist and found that technologist review was a cost-effective alternative to double reading by two radiologists. Torres-Mejía et al. ${ }^{5}$ found that technologists achieved similar sensitivity but had higher false-positive rates than U.S. radiologists.

In contrast to the study by Pauli et al, Duijm et al. ${ }^{4}$ found that secondary referral of technologist-positive readings was a successful strategy for increasing the cancer detection rate because of a high prevalence of breast cancer in this subset. In our study, only $3.4 \%$ of the interval cancers had had a technologist-positive screen for the same abnormality in a previous screening round. Moreover, only $0.2 \%$ of women who were not recalled at radiologist double reading but had a mammogram considered suspicious by the technologist were diagnosed with breast cancer. This suggests that technologist readings provide insufficient discriminating ability in deciding which negative screens at radiologist double reading are eligible for secondary referral. This is also in line with results from our previous study. ${ }^{9}$

Only a minority of additionally detectable cancers (sensitivity of $52 \%$ [26 of 50], $95 \%$ confidence interval: $38 \%, 66 \%$ ) were detected through our quality assurance sessions. The lack of significant differences in mammographic characteristics between 
the cancers detected after secondary referral through quality assurance sessions and the 128 cancers that were 'missed' provides no clues to the reason for this low sensitivity. A possible explanation could be the high cancer detection rate and programme sensitivity. An alternative strategy might be to refer all women with a positive screening result according to either radiologists or technologists; this would, however, nearly double our recall rate (from $3.0 \%$ to $5.5 \%$ ), which is undesirable, taking into account the European guidelines. ${ }^{14}$ In the future, artificial intelligence (AI) might have a role. In a recent study, Rodríguez-Ruiz et al. ${ }^{15}$ found that radiologists improved their diagnostic performance in breast cancer detection by using an $\mathrm{Al}$ computer system for support. In a different study, Rodríguez-Ruiz et al. ${ }^{16}$ found a similar cancer detection accuracy between stand-alone $\mathrm{Al}$ and radiologists. Their findings also suggest that the Al system improves the evaluation of equivocal cases. ${ }^{15}$ This would make it a possible tool in arbitrating discordant readings between two readers or even for replacing one of the radiologists in double reading. In our quality assurance sessions, Al might review technologist-positive screens alongside the coordinating screening radiologist. However, further research is needed to study the effects of incorporating $\mathrm{Al}$ in population-based breast cancer screening programmes, especially on recall rate, cancer detection rate, and other performance parameters.

Our study had a few limitations. The design of the Dutch nationwide breast cancer screening programme differs from that of other programmes. Our results are therefore mainly relevant to screening programmes that are similar to ours, and extrapolation to other screening programmes is limited. However, the lack of significant differences in mammographic characteristics between cancers detected through radiologist double reading, those detected after secondary recall through quality assurance sessions, and those that were initially missed is an interesting finding, irrespective of screening programme set-up. Further research is needed to answer the question of what makes radiologists "miss" these cancers. In our study, technologists had a lower cancer detection rate than did radiologists. However, screening radiologists were not blinded to the technologists' reading, reading conditions differed between both groups, and technologists performed single reading as opposed to the radiologists' double reading. This means we could not compare reading performance between technologists and radiologists, but this was not the purpose of our study. Because of our study design (specifically the fact that the radiologists were not blinded to the technologists reading), the value of the technologists' reading and quality assurance sessions could be underestimated as a result of bias. Because of small subgroup analysis, statistical power was limited.

In conclusion, the role of quality assurance sessions in additional cancer detection is limited. The number of extra cancers detected through these sessions was small $(<1 \%)$, and the mammographic and tumour characteristics of these cancers did not 
differ significantly compared with those of cancers detected at radiologist double reading. However, these sessions serve many other purposes, such as continued training of technologists (in lesion detection, characterisation, and classification), discussing interesting and instructive cases, and receiving feedback on performance. 


\section{References}

1. International Agency for Research on Cancer. Global Cancer Observatory (GCO). http://gco.iarc.fr. Accessed June 19, 2019.

2. Netherlands Comprehensive Cancer Organisation. Cijfers over Kanker. http://www.cijfersoverkanker.nl. Accessed June 19, 2019.

3. Sankatsing VDV, van Ravesteyn NT, Heijnsdijk EAM, et al. The effect of population based mammography screening in Dutch municipalities on breast cancer mortality: 20 years of follow-up. Int J Cancer 2017;141(4):671-677.

4. Duijm LEM, Groenewoud JH, Fracheboud J, de Koning HJ. Additional double reading of screening mammograms by radiologic technologists: impact on screening performance parameters. J Natl Cancer Inst 2007;99(15):1162-1170.

5. Torres-Mejía G, Smith RA, Carranza-Flores ML, et al. Radiographers supporting radiologists in the interpretation of screening mammography: a viable strategy to meet the shortage in the number of radiologists. BMC Cancer 2015;15(1):410.

6. Duijm LEM, Louwman MWJ, Groenewoud JH, van de Poll-Franse LV, Fracheboud J, Coebergh JW. Inter-observer variability in mammography screening and effect of type and number of readers on screening outcome. Br J Cancer 2009;100(6):901-907.

7. Pauli R, Hammond S, Cooke J, Ansell J. Comparison of radiographer/radiologist double film reading with single reading in breast cancer screening. J Med Screen 1996;3(1):18-22.

8. Tonita JM, Hillis JP, Lim CH. Medical radiologic technologist review: effects on a population-based breast cancer screening program. Radiology 1999;211(2):529-533.

9. Coolen AMP, Lameijer JRC, Voogd AC, et al. Incorporation of the technologist's opinion for arbitration of discrepant assessments among radiologists at screening mammography. Breast Cancer Res Treat 2018;171(1):143-149.

10. Duijm LEM, Groenewoud JH, Hendriks JHCL, de Koning HJ. Independent double reading of screening mammograms in the Netherlands: effect of arbitration following reader disagreements. Radiology 2004;231(2):564-570.

11. Klompenhouwer EG, Duijm LEM, Voogd AC, et al. Re-attendance at biennial screening mammography following a repeated false positive recall. Breast Cancer Res Treat 2014;145(2):429-437.

12. D'Orsi C, Sickles E, Mendelson E, et al. ACR BI-RADS Atlas, Breast Imaging Reporting and Data System. Reston, Va: American College of Radiology, 2013.

13. van Bommel RMG, Weber R, Voogd AC, et al. Interval breast cancer characteristics before, during and after the transition from screen-film to full-field digital screening mammography. BMC Cancer 2017;17(1):315.

14. European Commission. European guidelines for quality assurance in breast cancer screening and diagnosis. 4th ed. Luxembourg: Office for Official Publications of the European Communities, 2013.

15. Rodríguez-Ruiz A, Krupinski E, Mordang JJ, et al. Detection of breast cancer with mammography: effect of an artificial intelligence support system. Radiology 2019;290(2):305-314.

16. Rodríguez-Ruiz A, Lång K, Gubern-Merida A, et al. Stand-alone artificial intelligence for breast cancer detection in mammography: comparison with 101 radiologists. J Natl Cancer Inst 2019;111(9):916922. 



\section{Chapter 5}

Frequency and characteristics of additional breast abnormalities following recall at screening mammography 



\section{Chapter 5.1}

Frequency and characteristics of contralateral breast abnormalities following recall at screening mammography

Joost R.C. Lameijer, Angela M.P. Coolen, Adri C. Voogd, Luc J. Strobbe, Marieke W.J. Louwman, Dick Venderink, Vivianne C.G. Tjan-Heijnen, Lucien E.M. Duijm European Radiology 2018;28:4205-4214 


\section{Abstract}

\section{Purpose}

To determine the frequency and characteristics of contralateral, non-recalled breast abnormalities following recall at screening mammography.

\section{Methods}

We included a series of 130,338 screening mammograms performed between 1 January 2014 and 1 January 2016. During the 1-year follow-up, clinical data were collected for all recalls. Screening outcome was determined for recalled women with or without evaluation of contralateral breast abnormalities.

\section{Results}

Of 3995 recalls (recall rate 3.1\%), 129 women (3.2\%) underwent assessment of a contralateral, non-recalled breast abnormality. Most lesions were detected at clinical mammography and/or breast tomosynthesis (101 women, 78.3\%). The biopsy rate was similar for recalled lesions and contralateral, non-recalled lesions, but the positive predictive value of biopsy was higher for recalled lesions $(p=0.01)$. A comparable proportion of the recalled lesions and contralateral, non-recalled lesions were malignant $(p=0.1)$. The proportion of ductal carcinoma in situ was similar for both groups, as well as invasive cancer characteristics and type of surgical treatment.

\section{Conclusions}

About $3 \%$ of recalled women underwent evaluation of contralateral, non-recalled breast lesions. Evaluation of the contralateral breast after recall is important as we found that $15.5 \%$ of contralateral, non-recalled lesions were malignant. Contralateral cancers and screendetected cancers show similar characteristics, stage and surgical treatment. 


\section{Introduction}

Many countries have introduced breast cancer screening programmes with the aim to detect breast cancers at an early stage, before these grow large enough to become symptomatic, and thus to decrease morbidity and improve breast cancer survival. Breast cancer mortality has decreased in the past 20 years in The Netherlands and this improved survival is due to a combination of breast cancer screening and improved treatment. $^{1-3}$

Breast cancers detected at screening are often small and non-symptomatic. Improved radiological breast imaging techniques, including the replacement of screen-film mammography by full-field digital mammography (FFDM) and the recent introduction of breast tomosynthesis (three-dimensional mammography), have resulted in a more accurate detection and characterisation of breast lesions at screening mammography. ${ }^{4,5}$ Breast tomosynthesis has not yet been implemented in our nationwide screening mammography programme. Women are usually recalled for a unilateral lesion at screening mammography, but a small percentage of cases (1-2\%) is recalled for suspicious bilateral lesions. ${ }^{6}$

After recall, additional breast imaging is performed for the evaluation of the suspected lesion detected at screening mammography. In a diagnostic setting, the addition of breast tomosynthesis to digital mammography results in a higher sensitivity and specificity of breast cancer diagnosis than digital mammography alone. ${ }^{7-9}$ For example, Gilbert et al. ${ }^{9}$ reported a higher sensitivity for the detection of invasive cancers sized $11-20 \mathrm{~mm}$ when adding tomosynthesis to digital mammography in a diagnostic setting (93\% versus $86 \%$, $p<0.001$ ). Specificity also increased, from $58 \%$ with digital mammography to $69 \%$ with digital mammography plus breast tomosynthesis. Lång et al. ${ }^{10}$ found an increased lesion visualisation with breast tomosynthesis compared to digital mammography, especially for spiculated masses.

Additional breast cancers may be detected in the breast for which the woman has been recalled, or in the contralateral breast. Preoperative breast magnetic resonance imaging (MRI) in breast cancer patients has also been shown to detect additional malignancies in one or both breasts in a significant number of patients, and the MRI findings frequently result in more extensive surgery compared to the initially proposed treatment, such as additional contralateral mastectomy. ${ }^{11-13}$

To our knowledge, no previous studies have reported on additionally detected contralateral breast lesions in women recalled for a unilateral abnormality at screening mammography. We, therefore, assessed the frequency and characteristics 
of these contralateral lesions in women who attended a biennial screening mammography programme in the south of the Netherlands.

\section{Materials and methods}

\section{Study design end study population}

This is a prospective observational follow-up study of women aged 50-75 who attended a biennial breast cancer screening programme conducted in the south of The Netherlands. A consecutive series of 130,338 full-field digital mammography screens (13,762 initial screens and 116,576 subsequent screens) between 1 January 2014 and 1 January 2016 were included. The screening mammograms were obtained at four specialised screening units in a biennial screening mammography programme conducted in the south of The Netherlands.

Women are personally invited by letter to attend the screening programme. These letters are sent to the address of every woman aged between 50 and 75 years old registered in the municipal registration. Women being treated for breast cancer or those attending clinical follow-up after treatment of breast cancer do not attend the screening programme. Otherwise, there are no exclusion criteria for screening eligibility.

Women participating in our screening mammography programme were asked for permission to use their data for scientific purposes and for the evaluation of the screening programme. All women gave permission to use their screening and diagnostic data.

Possible exclusion criteria included no permission to use screening data, technical failure of screening equipment and insufficient image quality as assessed by an experienced screening radiologist. None of the women screened for study inclusion met these criteria.

A total of 3995 women were recalled for further analysis. The hospitals involved in these recalls were visited and data as described below were obtained. Possible exclusion criteria after recall included insufficient follow-up or loss to follow-up, incomplete records, no permission to access data. None of the women recalled for analysis met these criteria and no recalls were excluded from analysis.

Ethical approval by our local Institutional Review Board was not required for this prospective observational follow-up study, according to the Dutch Central Committee on Research involving Human Subjects (CCMO). 


\section{Screening procedure and recall}

Details of our breast cancer screening programme have been described previously. ${ }^{14}$ In brief, the mammography screening programme in The Netherlands is a nationwide programme that provides free biennial screening mammography for women aged 50 75 years. Women are personally invited for screening and the attendance rate is more than $80 \%$. Before each screening examination, the woman completes a short questionnaire with questions about previous recalls and any previous breast surgery or breast malignancy.

All digital mammograms were obtained with a Lorad Selenia FFDM system (Hologic, Danbury, CT, USA), with a 70- $\mu \mathrm{m}$ pixel size and a $232 \times 286-\mathrm{mm}$ field of view. The examinations were obtained by specialised screening mammography technologists and all screening mammograms were double-read in a blinded fashion by a team of 12 certified screening radiologists. Each radiologist evaluated at least 3,000 mammograms yearly.

The screening radiologists classified abnormal mammographic findings into one of the following categories: suspicious mass, suspicious calcifications, suspicious mass in combination with calcifications, architectural distortion, asymmetry or other. Each screen was then classified according to the BI-RADS lexicon. ${ }^{15}$ Women with a BI-RADS 1 or 2 were not recalled and women with a BI-RADS 0,4 or 5 were referred to a dedicated breast unit of a hospital for further analysis of their mammographic finding. The BI-RADS category 3 is not applied as short-term follow-up is not available in the Dutch screening programme.

\section{Diagnostic work-up after recall}

Although a total of 20 hospitals were involved in the diagnostic work-up of recalled women, the diagnostic work-up in the majority of women $(97.2 \%, 3,883 / 3,995)$ was performed in seven regional hospitals. All recalled women underwent physical examination by a surgical oncologist or dedicated breast nurse and received additional evaluation at the radiology department. A radiologist first reassessed the screening mammogram of both breasts, which was routinely available and stored in the Picture Archiving and Communication System (PACS) of the hospital. Additional imaging was obtained at the discretion of the radiologist after this review. Full-field digital mammography was available in each of the seven regional hospitals. Breast tomosynthesis was present in two hospitals from the beginning of the inclusion period and became available in three others in 2015. Breast ultrasonography was used for the further characterisation of mammographic abnormalities and palpable breast lesions, for biopsy guidance and for target or second look purposes following breast MRI. Whole-breast ultrasonography was not encouraged, in accordance with the Dutch guidelines. ${ }^{16}$ Breast MRI was also available in each of the seven hospitals and 
performed if indicated, as defined by the guidelines of the European Society of Breast Imaging ${ }^{17}$ and the Dutch guidelines. Fine-needle aspiration biopsy (FNAB), core-needle biopsy (CNB) and stereotactic biopsy were performed in each of the seven hospitals, whereas MRI guided biopsy procedures were concentrated in the larger hospitals. All recalls were discussed by multidisciplinary teams that consisted of surgical oncologists, radiologists, medical oncologists, radiation oncologists, plastic surgeons, breast nurses and breast technologists.

During 1-year follow-up, clinical data and data from diagnostic breast imaging, biopsy specimen and surgical procedures were collected of all recalled women. Information on breast density, hormonal replacement therapy and family history of breast cancer were also extracted from the screening records and clinical data. Breast cancers were categorised into ductal carcinoma in situ (DCIS) and invasive cancers; lobular carcinoma in situ was considered a non-malignant lesion. The TNM (tumour, nodes and metastases) classification was used for malignant lesions. ${ }^{18}$ For all cancers treated by neoadjuvant therapy (either chemotherapy or anti-hormonal therapy), tumour size was derived from breast imaging (usually MRI) prior to the start of this therapy.

\section{Detection and assessment of contralateral breast lesions}

The methods of detection and subsequent assessment of contralateral breast lesions were derived from the clinical records and clinical radiology reports. Similar to the work-up of screen-detected mammographic abnormalities, biopsy data and surgical reports of contralateral breast lesions were obtained.

Two screening radiologists reviewed the latest screening mammogram of each woman with a cancer diagnosed in the contralateral breast. They classified the cancer as missed, minimal sign or occult at the previous screen according to the European guidelines. ${ }^{19,20}$

\section{Statistical analysis, missing data and bias}

Descriptive statistics were performed using Statistical Package for Social Science 23.0 (SPSS IBM, Chicago, IL, USA). The chi-squared test was used to test for differences between women without and with additional contralateral breast lesions detected following recall. A p-value of less than 0.05 was considered to indicate a statistically significant difference. The $p$-values were two sided.

There was no loss to follow-up. In a small number of cases, data were missing (e.g. oestrogen or progesterone receptor status missing due to an insufficient tissue sample). Statistical correction was not performed for the very small numbers of missing data, and data missing was considered to be at random. 
Bias was not expected in recall of women who attended screening. In the diagnostic setting, several types of bias may occur, especially observer bias and detection bias, but statistical correction for this type of bias is not feasible in this study. Selection bias was considered highly unlikely as all women analysed in the diagnostic setting were included for statistical analysis.

\section{Results}

\section{Overall screening results}

The mean age of the 130,338 consecutively screened women was 59.6 years $(95 \% \mathrm{Cl}$, 59.4-59.8 years) and $8.2 \%$ reported to have had previous breast surgery. A total of 3995 (3.1\%) were recalled for further assessment. Breast cancer was diagnosed in 905 recalled women [including $163(18.0 \%)$ ductal carcinoma in situ], resulting in an overall cancer detection rate of 6.9 per 1000 screens [initial screens, $8.6(118 / 13,762)$; subsequent screens, $6.8(787 / 116,576)]$ and an overall positive predictive value (PPV) of recall of $22.7 \%$ [initial screens, $11.4 \%$ (118/1037); subsequent screens, $26.6 \%$ (787/2958)]. A patient flow chart is provided in Figure 5.1.1.

\section{Detection of contralateral lesions}

A total of 129 women, 3.2\% of all recalls, underwent assessment of a contralateral breast abnormality that was detected after recall. Baseline characteristics of recalled women, with or without analysis of a contralateral non-recalled lesion, are shown in Table 5.1.1. These characteristics were similar for both groups except for a higher breast density (III + IV) in women assessed for a contralateral breast lesion when compared to a recalled breast lesion ( $38.8 \%$ and $23.8 \%$ respectively, $p<0.001$ ). The contralateral abnormality was detected at clinical mammography and/or breast tomosynthesis in 101 women and at breast MRI in 23 women. In the remaining five women, the contralateral breast was evaluated due to the presence of a suspicious finding at clinical breast examination by the surgical oncologist or breast nurse (palpable breast lesion, four cases; Paget disease of the nipple, one case). 


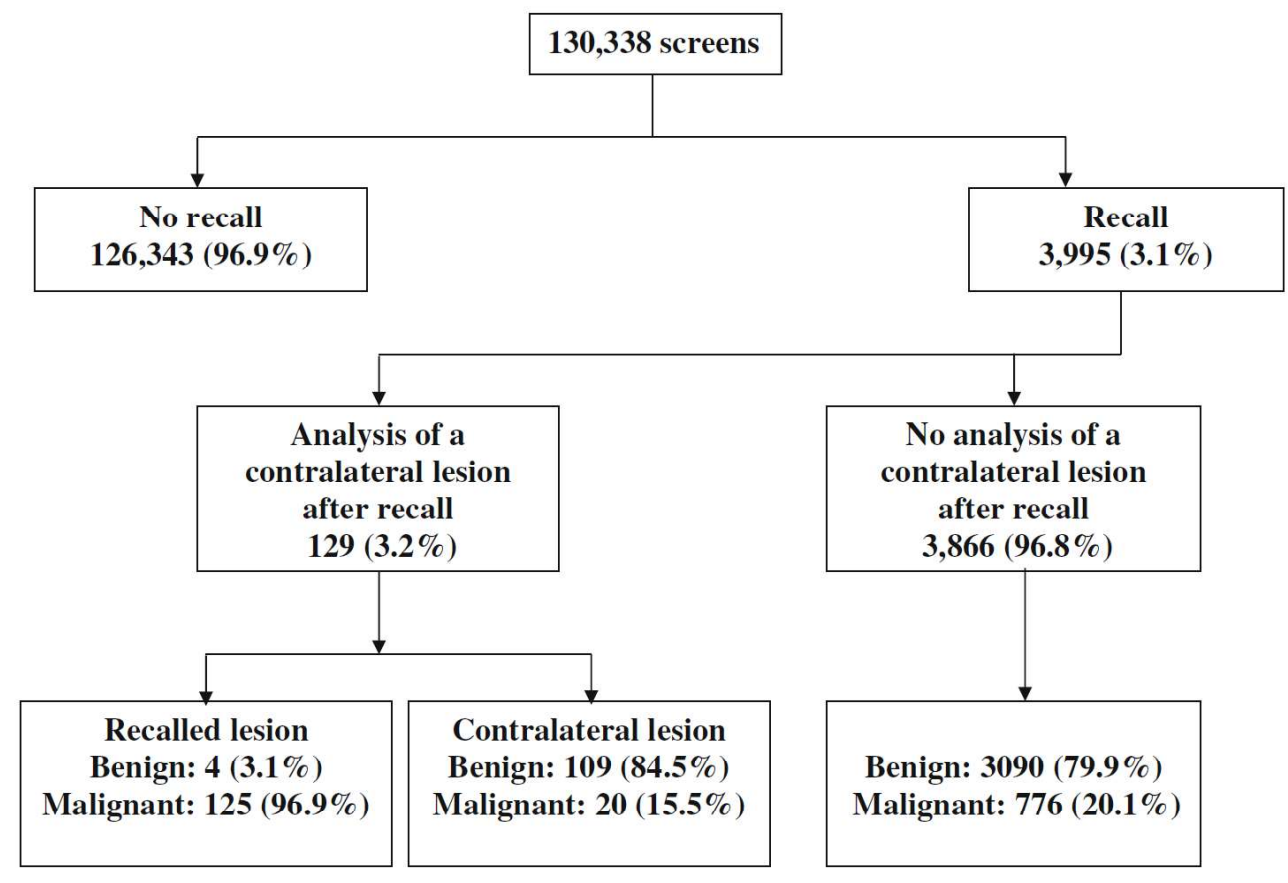

Figure 5.1.1 Flow chart of study population.

Table 5.1.1 Baseline characteristics of recalled women.

\begin{tabular}{|c|c|c|c|}
\hline & $\begin{array}{c}\text { No contralateral } \\
\text { lesions } \\
\mathrm{N}=3866\end{array}$ & $\begin{array}{l}\text { Contralateral Lesions } \\
\qquad \mathrm{N}=129\end{array}$ & P-value \\
\hline Mean age, years $(95 \% \mathrm{Cl})$ & $59.1(58.8-59.4)$ & $58.4(57.1-59.8)$ & 0.35 \\
\hline Screening round, No (\%) & & & 0.13 \\
\hline Initial & $996(25.8)$ & $41(31.8)$ & \\
\hline Subsequent & $2870(74.2)$ & $88(68.2)$ & \\
\hline Breast density, No (\%) & & & $<0.001$ \\
\hline$|+| \mid(\leq 50 \%)$ & $2946(76.2)$ & $79(61.2)$ & \\
\hline $\mathrm{III+IV}(>50 \%)$ & $920(23.8)$ & $50(38.8)$ & \\
\hline Previous breast surgery, No (\%) & & & 0.69 \\
\hline Yes & $322(8.3)$ & $12(9.3)$ & \\
\hline No & $3544(91.7)$ & $117(90.7)$ & \\
\hline Hormone replacement therapy, No (\%) & & & 0.11 \\
\hline Yes & $159(4.1)$ & $9(7.0)$ & \\
\hline No & 3707 (95.9) & $120(93.0)$ & \\
\hline Family history of breast cancer, No (\%) & & & 0.61 \\
\hline Yes & $509(13.2)$ & 19 (14.7) & \\
\hline No & $3357(86.8)$ & $110(85.3)$ & \\
\hline
\end{tabular}

The mammographic characteristics of the contralateral lesions detected at clinical mammography (with/without tomosynthesis) were as follows: suspicious mass, 90; suspicious calcifications, 8; suspicious mass with calcifications, 1; architectural distortion, 2. The indications for breast MRI in the 23 women with a contralateral 
lesion detected at MRI had been as follows: problem solving of the recalled lesion, 9 women; determination of tumour size and/or multifocality/multicentricity of a screendetected malignancy, 10 women; invasive lobular cancer, 4 women. Breast MRI showed a suspicious mass or non-mass enhancement in 19 women and 4 women, respectively.

\section{Work-up of lesions detected in the contralateral breast}

The majority of women with an abnormality detected in the contralateral breast underwent only breast imaging for the further evaluation of these lesions (Table 5.1.2). A total of 74 women (57.4\%) received breast ultrasonography and the remaining 55 women (42.6\%) underwent one or several percutaneous and/or excisional biopsy procedures to establish a final pathological diagnosis.

Table 5.1.2 Workup of lesions detected in the contralateral breast during recall

\begin{tabular}{lc}
\hline Breast ultrasonography, No (\%) & $\mathbf{7 4}(\mathbf{5 7 . 4 )}$ \\
\hline Breast ultrasonography plus biopsy, No (\%) & $1(0.8)$ \\
FNAC & $36(27.9)$ \\
CNB & $10(7.8)$ \\
SCNB & $1(0.8)$ \\
FNAC+CNB & $2(1.6)$ \\
CNB+SCNB & $4(3.1)$ \\
Percutaneous biopsy + excisional biopsy & $1(0.8)$ \\
Excisional biopsy & \\
\hline
\end{tabular}

$F N A C=$ fine needle aspiration cytology; CNB = core needle biopsy; SCNB = stereotactic core needle biopsy

\section{Outcome of lesions detected in the contralateral breast}

Of the 129 lesions detected in the contralateral breast, 20 (15.5\%) proved to be malignant (Table 5.1.3). The majority of the malignancies presented themselves as a suspicious mass at clinical mammography ( 8 cases, $47.1 \%$ ) or breast MRI 7 cases, $41.2 \%)$. Of the nine contralateral breast cancers detected at mammography, two were visible only at breast tomosynthesis and not at the full-field digital mammogram. Of the seven cancers visible at the latest screening mammogram in retrospect, four were considered to be missed and three showed a minimal sign, respectively. As mentioned previously, the remaining three contralateral cancers presented as palpable breast lesions, not primarily detected at breast imaging. In 16 of the 20 women with a breast cancer diagnosed in the contralateral breast, the mammographic abnormality at screening turned out to be malignant as well, resulting in 16 bilateral cancer cases. In the four remaining cases, the contralateral lesion turned out to be malignant, and the mammographic abnormality at screening turned out to be benign. One of these cases is presented in Figure 5.1.2. The majority of benign lesions detected in the 
contralateral breast comprised cysts $(52.3 \%, 57 / 109)$ and fibroadenomas $(13.8 \%$, 15/109).

Table 5.1.3 Characteristics and outcome of lesions in the contralateral breast, detected at clinical breast imaging following recall.

\begin{tabular}{lcc}
\hline & \multicolumn{2}{c}{ Final outcome } \\
Detection method and radiological abnormality, No (\%) & Benign & Malignant \\
\hline Mammography & & $6(35.3)$ \\
$\quad$ Suspicious mass & $81(65.2)$ & $1(5.9)$ \\
Suspicious calcifications & $8(6.5)$ & 0 \\
Suspicious mass with calcifications & $1(0.8)$ & 0 \\
Architectural distortion & $2(1.6)$ & $2(11.8)$ \\
Tomosynthesis-only & $9(7.3)$ & $7(41.2)$ \\
$\quad$ Suspicious mass & & 1 \\
Breast MRI & $19(15.3)$ & $(5.9)$ \\
$\quad$ Suspicious mass & $4(3.2)$ & \\
$\quad$ Focal non-mass enhancement & & \\
\hline
\end{tabular}

${ }^{ \pm}$With or without breast tomosynthesis

Comparison of unilateral, screen-detected abnormalities with those detected in the contralateral, non-recalled breast

Of the recalled lesions and contralateral, non-recalled lesions, respectively $22.6 \%$ and $15.5 \%$ proved to be malignant ( $p=0.06$; Table 5.1.4). The type of assessment after recall (clinical breast imaging versus biopsy in addition to clinical breast imaging) was similar for both groups. The positive predictive value of biopsy, however, was higher for recalled lesions $(53.1 \%$ vs. $36.4 \%, p=0.01)$. The tumour characteristics and final surgical treatment of unilateral, screen-detected cancers were similar to those of cancers diagnosed in the contralateral, non-recalled breast (Table 5.1.5). 

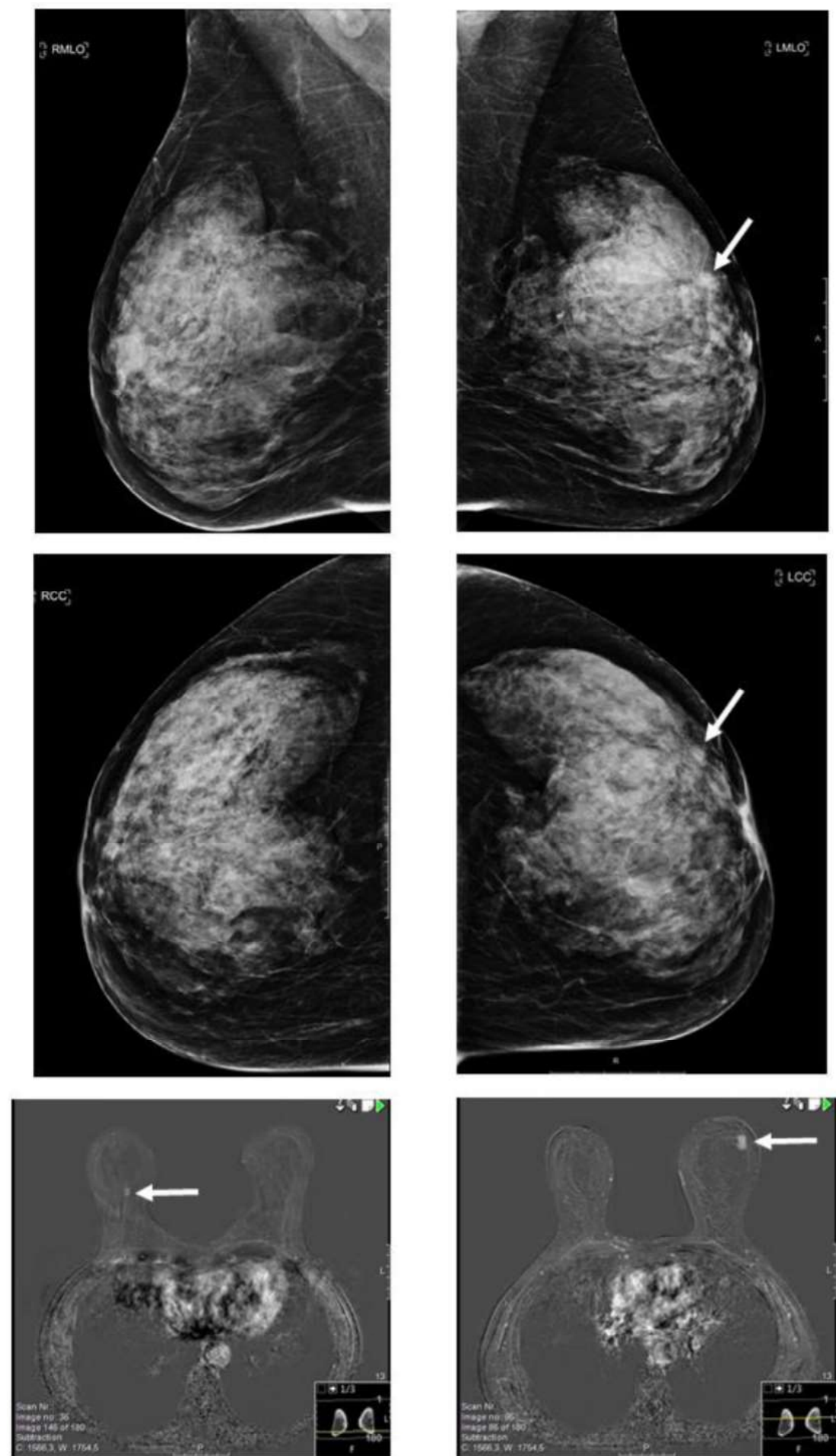

Figure 5.1.2 A 64-year-old woman was recalled for a BI-RADS 4 mass in the left breast. Percutaneous core biopsy showed invasive ductal carcinoma and MRI was performed to determine the extent of the disease. MRI demonstrated a BIRADS 4 mass in the right breast, which was occult at subsequent target ultrasound. MRI guided vacuum assisted biopsy revealed invasive lobular carcinoma. Breast conserving surgery yielded and invasive ductal carcinoma of $8 \mathrm{~mm}$ in the left breast (sentinel node negative, B\&R II, ER+, PR+, Her2-) and an invasive lobular carcinoma of $6 \mathrm{~mm}$ in the right breast (sentinel node negative, B\&R I, ER+, PR-, Her2-). 
Table 5.1.4 Overall screening outcome of recalled lesions versus lesions analysed in the contralateral, non-recalled breast.

\begin{tabular}{lccc}
\hline & $\begin{array}{c}\text { Recalled } \\
\text { lesions } \\
\mathbf{N = 3 9 9 5}\end{array}$ & $\begin{array}{c}\text { Lesions assessed in the } \\
\text { contralateral, non-recalled breast } \\
\mathbf{N = 1 2 9}\end{array}$ & P-value \\
\hline $\begin{array}{l}\text { Screening outcome, No (\%) } \\
\quad \text { False positive }\end{array}$ & $3094(77.4)$ & $109(84.5)$ & 0.06 \\
$\quad \begin{array}{l}\text { True positive } \\
\text { Type of assessment after recall, No (\%) }\end{array}$ & $901(22.6)$ & $20(15.5)$ & 0.98 \\
$\quad$ Clinical breast imaging & $2297(57.5)$ & $74(57.4)$ & 0.01 \\
$\quad$ Clinical breast imaging + biopsy & $1698(42.5)$ & $55(42.6)$ & 36.4 \\
Positive predictive value of biopsy, \% & 53.1 & & 0.06 \\
\hline
\end{tabular}

${ }^{ \pm}$Dominant mammographic abnormality in case of multiple recalled lesions.

Table 5.1.5 Tumour characteristics of unilateral, screen-detected cancers versus cancers diagnosed in the contralateral, non-recalled breast.

\begin{tabular}{|c|c|c|c|}
\hline & $\begin{array}{c}\begin{array}{c}\text { Unilateral SDCs } \\
(n=901)\end{array} \\
\end{array}$ & $\begin{array}{l}\text { Contralateral cancers } \\
(n=20)\end{array}$ & P-value \\
\hline Type of cancer, No (\%) & & & 0.4 \\
\hline DCIS & $162(18.0)$ & $2(10.0)$ & \\
\hline Invasive & $739(82.0)$ & $18(90.0)$ & \\
\hline Histology of invasive cancers, No (\%) & & & 0.2 \\
\hline Ductal & $581(78.6)$ & $12(66.7)$ & \\
\hline Lobular & $91(12.3)$ & $5(27.8)$ & \\
\hline Ductolobular & $23(3.1)$ & 0 & \\
\hline Other & $44(6.0)$ & $1(5.6)$ & \\
\hline Tumour stage of invasive cancers, No (\%) & & & 0.97 \\
\hline $\mathrm{T} 1$ & $569(77.0)$ & $14(77.8)$ & \\
\hline $\mathrm{T} 2+$ & 169 (22.9) & $4(22.2)$ & \\
\hline Unknown & $1(0.1)$ & 0 & \\
\hline Lymph node status of invasive cancers, No (\%) & & & 0.7 \\
\hline $\mathrm{N}+$ & $164(22.2)$ & $5(27.8)$ & \\
\hline $\mathrm{N}-$ & $561(76.0)$ & $13(72.2)$ & \\
\hline Unknown & $14(1.9)$ & 0 & \\
\hline Bloom \& Richardson grade, No (\%) & & & 0.8 \\
\hline 1 & $329(44.5)$ & $8(44.4)$ & \\
\hline II & $318(43.0)$ & $9(50.0)$ & \\
\hline III & $87(11.8)$ & $1(5.6)$ & \\
\hline Unknown & $5(0.7)$ & 0 & \\
\hline Estrogen receptor status, No (\%) & & & 0.4 \\
\hline Positive & $667(90.3)$ & $18(100)$ & \\
\hline Negative & $69(9.3)$ & 0 & \\
\hline Unknown & $3(0.4)$ & 0 & \\
\hline Progesterone receptor status, No (\%) & & & 0.5 \\
\hline Positive & $520(70.4)$ & $15(83.3)$ & \\
\hline Negative & $216(29.2)$ & $3(16.7)$ & \\
\hline Unknown & $3(0.4)$ & 0 & \\
\hline Her2/Neu receptor status, No (\%) & & & 0.8 \\
\hline Positive & 72 (9.7) & $1(5.6)$ & \\
\hline Negative & $663(89.7)$ & $17(94.4)$ & \\
\hline Unknown & $4(0.5)$ & 0 & \\
\hline Final surgical treatment, No (\%) & & & 0.9 \\
\hline Breast conserving surgery & $726(80.6)$ & $17(85.0)$ & \\
\hline Mastectomy & $163(18.1)$ & $3(15.0)$ & \\
\hline No surgery & $11(1.2)$ & 0 & \\
\hline Unknown & $1(0.1)$ & 0 & \\
\hline
\end{tabular}

DCIS = Ductal carcinoma in situ 


\section{Discussion}

In the current study, we found that one out of 30 recalled women underwent analysis of a contralateral, non-recalled breast lesion and about one out of seven of these latter lesions proved to be malignant. The positive predictive value of biopsy of contralateral abnormalities, detected following recall, was lower than that of screendetected lesions. Contralateral cancers and screen-detected cancers showed similar histological tumour characteristics, tumour stage and surgical treatment.

In retrospect, four out of 20 contralateral malignancies in our series were considered to be missed at the latest screening examination. Setz-Pels et al. ${ }^{6}$ found that the sensitivity of screening mammography for the detection of bilateral breast cancer is less than $20 \%$. The authors concluded that both screening radiologists and clinical radiologists should pay vigorous attention to the contralateral breast in order to detect bilateral malignancies without diagnostic delay. However, they do not provide information on the overall presence and work-up of contralateral, nonrecalled breast abnormalities. We found that $3.2 \%$ of recalled women underwent assessment of a contralateral, non-recalled breast abnormality. The majority of these lesions were detected at clinical mammography and/or breast tomosynthesis. Tomosynthesis is increasingly being used, both at screening mammography and in a diagnostic setting. The addition of tomosynthesis to digital screening mammography may increase the cancer detection rate. A recent US study reported a decreased recall rate 3 years after the introduction of breast tomosynthesis (from 10.4 to $9.0 \%$, $p<0.001$ ), as well as an increased positive predictive value of recall (from 4.4 to $6.7 \%$, $\mathrm{p}=0.02$ ) and a decreased interval cancer rate. A UK study found an increased specificity when adding tomosynthesis to digital screening mammography $(70 \%$ versus $57 \%, p<0.001)$ and an increased sensitivity for cancer detection in dense breasts $(93 \%$ versus $86 \%, p=0.03) .{ }^{9,21}$

Tomosynthesis is not yet implemented in the Dutch screening mammography setting. An increasing number of hospitals have this modality at their disposal. In our series, two of the contralateral breast cancers were visible only at breast tomosynthesis and not on the full-field digital mammogram. It is likely that the intensified use of tomosynthesis in the work-up of recalled women will increase the number of lesions detected in contralateral, non-recalled breasts. ${ }^{7,8}$ A substantial number of contralateral lesions in our study were detected at breast MRI. Again, it can be expected that a further increase in the use of MRI, as well as the introduction of new and promising imaging modalities such as contrast-enhanced spectral mammography ${ }^{22,23}$, will increase the number of breast lesions detected in the contralateral breast of recalled women. 
Additional percutaneous biopsy was performed in $43 \%$ of these lesions, with a lower positive predictive value of biopsy $(36.4 \%)$ than the one observed for recalled lesions (53.1\%, $\mathrm{p}<0.01)$. Excision biopsy, all with benign final pathology, was performed in five women. In some rare cases, excision biopsy may be necessary to establish a final diagnosis. Also, some women may desire excision of a benign lesion. Nevertheless, one should aim to minimise excision biopsy for diagnostic purposes as it has been shown that the sensitivity of screening mammography is lower in women after benign breast surgery. ${ }^{24}$

We found no statistically significant differences in the proportions of malignancies among recalled lesions and contralateral, non-recalled malignant lesions $(22.6 \%$ versus $15.5 \%$ ). This study could be statistically underpowered to detect a clinically relevant difference due to small patient numbers. The observed trend of a lower cancer risk for contralateral lesions may be due to the fact that screening radiologists have no other tool than the screening mammogram and they have to keep recall percentages within acceptable limits. On the other hand, clinical radiologists are likely to have a lower threshold to exclude malignancy in mammographic lesions for which a woman has not been recalled.

Diagnosis of bilateral breast cancer was established in 16 of 20 women with a contralateral malignant lesions in the non-recalled breast, and four unilateral cancers were diagnosed in a breast for which a woman had not been recalled. A synchronous diagnosis of bilateral breast cancer may have an impact on the choice of final surgical therapy as these women are more inclined to opt for mastectomy over breastconserving surgery. ${ }^{25}$ The survival of patients with bilateral disease is likely to be worse than that of patients with unilateral disease. ${ }^{26,27}$

Studies have demonstrated an improved cancer detection when MRI or wholebreast ultrasound is added to mammography. ${ }^{12,28,29}$ The increased sensitivity, however, is accompanied by an increase in false-positive findings. Moreover, enhanced use of MRI is correlated with an increase in mastectomy procedures. ${ }^{30-32}$ Therefore, the guidelines of the European Society of Breast Imaging and the Dutch guidelines do not promote the standard use of MRI or whole-breast ultrasound in recalled women or in women with pathologically proven breast cancer. ${ }^{16,17}$ Breast ultrasonography should be used mainly as an evaluation tool of lesions detected at mammography or MRI (target ultrasonography or second look) and for the assessment of palpable breast lesions that are occult at mammography. Indications for breast MRI are screening of high risk women, screening of women with dense breasts, detection of additional ipsilateral and contralateral malignancies in invasive lobular cancer, determination of tumour size in dense breasts and in select cases for problem solving of lesions detected at mammography and/or breast ultrasonography. 
Recalled women with or without assessment of a non-recalled, contralateral abnormality showed similar baseline characteristics except of a higher mammographic breast parenchyma density in the first group $(p<0.001)$. This may be explained by the fact that additional imaging modalities used in the clinical setting, as mentioned above, have a higher sensitivity for lesion detection compared to mammography, especially in dense breasts. ${ }^{10,12,29,30,33,34}$

It seems remarkable that three out of 20 detected contralateral malignancies were diagnosed by physical examination only. Contrary to a common perception that imaging obviates the need for physical examination, it remains a mainstay of diagnosis and an integral part of breast awareness and (self-)screening. ${ }^{35}$

Tumour characteristics of cancers diagnosed in the contralateral, non-recalled breast were similar to those of unilateral, screen-detected cancers. A majority of cancers in both groups were of the invasive ductal type, stage T1, lymph node negative and grade I or II. A Dutch study found that contralateral breast cancers detected by screening comprised more lobular cancers and showed less nodal involvement than index cancers or unilateral cancers. ${ }^{6}$

Our study has certain strengths and limitations. To our knowledge, we are the first to describe the frequency and characteristics of contralateral breast lesions following recall. The study population is large and recalled women were evaluated in multiple hospitals. Our results, however, may not be representative for other regional or nationwide screening mammography programmes as these programmes may show variations in screening interval (1-3 years), reading strategy (single reading versus double reading), percentage of recalled women and availability of higher-end radiological equipment (e.g. tomosynthesis, stereotactic core-needle biopsy, 3T MRI) at departments of radiology. Furthermore, as mentioned previously, the number of lesions detected in the contralateral breast may increase in the future as new techniques are increasingly implemented in the setting of clinical breast imaging. Ultimately, a majority of the women with contralateral breast lesions only underwent clinical breast imaging, without additional biopsy. BI-RADS 1 and BI-RADS 2 findings did not receive any radiological follow-up, whereas BI-RADS 3 lesions were either biopsied or received their first radiological follow-up at 6 months. As the follow-up period of all recalled women was 1 year, we cannot rule out that a contralateral lesion with benign follow-up may eventually turn out to be malignant.

In summary, reassessment of the complete screening mammogram, including the contralateral breast after recall, is important as contralateral cancers may be detected. We do not advocate a routine evaluation of the contralateral breast with additional imaging procedures following this reassessment, as this strategy will likely 
not be cost-effective. A timely diagnosis of the contralateral breast may be of influence on the choice of surgical therapy of a screen-detected cancer and survival. 


\section{References}

1. Sankatsing VDV, van Ravesteyn NT, Heijnsdijk EAM, et al. The effect of population-based mammography screening in Dutch municipalities on breast cancer mortality: 20 years of follow-up. Int J Cancer 2017;141:671-677.

2. Otto SJ, Fracheboud J, Looman CW, et al. Initiation of population-based mammography screening in Dutch municipalities and effect on breast-cancer mortality: a systematic review. Lancet 2003;361: 1411-1417.

3. Welch HG, Prorok PC, O'Malley AJ, Kramer BS. Breast cancer tumor size, overdiagnosis, and mammography screening effectiveness. N Engl J Med 2016;375:1438-447.

4. Pisano ED, Gatsonis C, Hendrick E, et al. Diagnostic performance of digital versus film mammography for breast-cancer screening. N Engl J Med 2005;353:1773-1783.

5. Bernardi D, Macaskill $P$, Pellegrini $M$, et al. Breast cancer screening with tomosynthesis (3D mammography) with acquired or synthetic 2D mammography compared with 2D mammography alone (STORM-2): a population-based prospective study. Lancet Oncol 2016;17:1105-1113.

6. Setz-Pels W, Duijm LE, Groenewoud JH, et al. Detection of bilateral breast cancer at biennial screening mammography in the Netherlands: a population-based study. Radiology 2011;260:357-363.

7. Waldherr C, Cerny P, Altermatt HJ, et al. Value of one-view breast tomosynthesis versus two-view mammography in diagnostic workup of women with clinical signs and symptoms and in women recalled from screening. AJR Am J Roentgenol 2013;200:226-231.

8. Lei J, Yang P, Zhang L, Wang Y, Yang K. Diagnostic accuracy of digital breast tomosynthesis versus digital mammography for benign and malignant lesions in breasts: a meta-analysis. Eur Radiol 2014;24: 595-602.

9. Gilbert FJ, Tucker L, Gillan MG et al. The TOMMY trial: a comparison of TOMosynthesis with digital MammographY in the UK NHS Breast Screening Programme-a multicentre retrospective reading study comparing the diagnostic performance of digital breast tomosynthesis and digital mammography with digital mammography alone. Health Technol Assess 2015;19:i-xxv 1-136.

10. Lang K, Andersson I, Zackrisson S. Breast cancer detection in digital breast tomosynthesis and digital mammography-a sideby- side review of discrepant cases. Br J Radiol 2014;87:20140080.

11. El Sharouni MA, Postma EL, Menezes GL, et al. High prevalence of MRI-detected contralateral and ipsilateral malignant findings in patients with invasive ductolobular breast cancer: impact on surgical management. Clin Breast Cancer 2016;16:269-275.

12. Brennan ME, Houssami N, Lord S, et al. Magnetic resonance imaging screening of the contralateral breast in women with newly diagnosed breast cancer: systematic review and meta-analysis of incremental cancer detection and impact on surgical management. J Clin Oncol 2009;27:5640-5649.

13. Wang SY, Long JB, Killelea BK, et al. Preoperative breast magnetic resonance imaging and contralateral breast cancer occurrence among older women with breast cancer. J Clin Oncol 2016;34:321-328.

14. Duijm LE, Groenewoud JH, Jansen FH, Fracheboud J, van Beek M, de Koning HJ. Mammography screening in the Netherlands: delay in the diagnosis of breast cancer after breast cancer screening. $\mathrm{Br}$ J Cancer 2004;91:1795-1799.

15. Sickles EA, D’Orsi CJ, Bassett LW, et al. ACR BI-RADS ${ }^{\circledR}$ Mammography. In: ACR BI-RADS ${ }^{\circledR}$ Atlas, Breast Imaging Reporting and Data System. American College of Radiology, Reston 2013.

16. NABON (2012) Richtlijn Mammacarcinoom. Available via http://www.oncoline.nl/mammacarcinoom. Accessed 11 July 2017

17. Mann RM, Balleyguier C, Baltzer PA, et al. Breast MRI: EUSOBI recommendations for women's information. Eur Radiol 2015;25:3669-78.

18. Sobin LH, Gospodarowicz MK, Wittekind C (eds). TNM classification of malignant tumours, 7th edn. Wiley, Chichester 2009.

19. Maes RM, Dronkers DJ, Hendriks JH, Thijssen MA, Nab HW. Do non-specific minimal signs in a biennial mammographic breast cancer screening programme need further diagnostic assessment? Br J Radiol 1997;70:34-38. 
20. Perry N, BroedersM, deWolf C, Tornberg S, Holland R, von Karsa L. European guidelines for quality assurance in breast cancer screening and diagnosis. Fourth edition-summary document. Ann Oncol 2008;19:614-622.

21. McDonald ES, Oustimov A, Weinstein SP, Synnestvedt MB, Schnall M, Conant EF. Effectiveness of digital breast tomosynthesis compared with digital mammography: outcomes analysis from 3 years of breast cancer screening. JAMA Oncol 2016;2:737-743.

22. Lobbes MB, Lalji U, Houwers J, et al. Contrast-enhanced spectral mammography in patients referred from the breast cancer screening programme. Eur Radiol 2014;24:1668-1676.

23. Lalji UC, Houben IP, Prevos R, et al. Contrast-enhanced spectral mammography in recalls from the Dutch breast cancer screening program: validation of results in a large multireader, multicase study. Eur Radiol 2016;26:4371-4379.

24. van Breest Smallenburg V, Duijm LE, Voogd AC, et al. Lower sensitivity of screening mammography after previous benign breast surgery. Int J Cancer 2012;130:122-128.

25. O'Brien JA, Ho A, Wright GP, et al. Breast-conserving surgery in bilateral breast cancer. Ann Surg Oncol 2015;22:3389-3396.

26. Heron DE, Komarnicky LT, Hyslop T, Schwartz GF,Mansfield CM. Bilateral breast carcinoma: risk factors and outcomes for patients with synchronous and metachronous disease. Cancer 2000;88:2739-2750

27. Schaapveld M, Visser O, Louwman WJ, et al. The impact of adjuvant therapy on contralateral breast cancer risk and the prognostic significance of contralateral breast cancer: a population based study in the Netherlands. Breast Cancer Res Treat 2008;110:189-197.

28. lacconi C, Galman L, Zheng J, et al. Multicentric cancer detected at breast MR imaging and not at mammography: important or not? Radiology 2016;279:378-384.

29. Melnikow J, Fenton JJ, Whitlock EP, et al. Supplemental screening for breast cancer in women with dense breasts: a systematic review for the U.S. Preventive Services Task Force. Ann Intern Med 2016;164:268-278.

30. Houssami N, Abraham LA, Onega T, et al. Accuracy of screening mammography in women with a history of lobular carcinoma in situ or atypical hyperplasia of the breast. Breast Cancer Res Treat 2014;145: 765-773.

31. Chandwani S, George PA, Azu M, et al. Role of preoperative magnetic resonance imaging in the surgical management of earlystage breast cancer. Ann Surg Oncol 2014;21:3473-3480.

32. Vriens $\mathrm{IJH}$, Keymeulen $\mathrm{K}$, Lobbes $\mathrm{MBI}$, et al. Breast magnetic resonance imaging use in patients undergoing neoadjuvant chemotherapy is associated with less mastectomies in large ductal cancers but not in lobular cancers. Eur J Cancer 2017;81:74-80.

33. Sprague BL, Stout NK, Schechter C, et al. Benefits, harms, and cost-effectiveness of supplemental ultrasonography screening for women with dense breasts. Ann Intern Med 2015;162:157-166.

34. Lee $\mathrm{Cl}$, Cevik M, Alagoz O, et al. Comparative effectiveness of combined digital mammography and tomosynthesis screening for women with dense breasts. Radiology 2015;274:772-780.

35. Provencher L, Hogue JC, Desbiens C, et al. Is clinical breast examination important for breast cancer detection? Curr Oncol 2016;23:e332-339. 


\section{Chapter 5.2}

Frequency and characteristics of additionally detected ipsilateral breast lesions following recall at screening mammography

Joost R.C. Lameijer, Angela M.P. Coolen, Joost Nederend, Adri C. Voogd, Vivianne C.G. Tjan-Heijnen, Lucien E.M. Duijm 


\section{Abstract}

\section{Purpose}

To determine the frequency and outcome of additionally detected ipsilateral breast abnormalities following recall at screening mammography.

\section{Methods and materials}

We included a consecutive series of 130,338 screening mammograms obtained between January 1, 2014 and January 1, 2016. During 2-year follow-up, clinical data were collected of all recalls. Women with a bilateral recall (115) and women recalled for multiple lesions in one breast (165) were excluded from the analyses. Screening outcome parameters were determined for recalled women with or without evaluation of additional ipsilateral breast abnormalities following recall.

\section{Results}

A total of 3995 women were recalled (recall rate, 3.1\%). In 258 (6.4\%) of these women, another lesion was detected in the ipsilateral breast than the one for which she had been recalled. Biopsy was more frequently performed of additionally detected ipsilateral lesions than of recalled lesions $(55.8 \%(144 / 258))$ versus $39.7 \%(1375 / 3457),(p<0.001))$. The proportion of malignancy in recalled lesions and additionally detected lesions was comparable $(21.5 \%$ (743/3457) versus $19.0 \%$ (49/258), $p=0.34)$. Of all 144 biopsies of additionally detected ipsilateral lesions, 9 revealed a synchronous tumour in addition to a malignant recalled lesion, and 33 biopsies revealed multicentric or multifocal tumours. In 5 women, the recalled lesion turned out to be benign, whereas the additional lesion in a different quadrant was malignant at biopsy. A total of 97 biopsies showed benign findings.

\section{Conclusion}

A substantial proportion of women are analysed for additional ipsilateral breast lesions following recall. These lesions are more frequently biopsied than recalled lesions, but have a comparable probability of being malignant. The majority of additionally detected cancerous lesions are part of multifocal or multicentric malignancies. 


\section{Introduction}

Screening mammography programmes have successfully been implemented in western countries over the past decades. Several studies have observed a reduction in breast cancer mortality, following the introduction of breast cancer screening, which is explained by detection of breast malignancy in an earlier stage and improved treatment. ${ }^{1-3}$

Over the years, improvements in breast imaging have increased the accuracy and detection rates at screening mammography. There is ongoing research to increase the detection of malignant breast lesions and to reduce false positive recalls of screening mammography programmes, for example by implementation of breast tomosynthesis. ${ }^{2-11}$ In the diagnostic setting, additional imaging is usually performed to characterize a recalled lesion and additional ipsilateral or contralateral breast abnormalities may be detected. These additionally detected lesions are most frequently benign, but they also comprise malignant satellite lesions associated with the primary cancer and synchronous primary malignancies. ${ }^{12-16}$ Few results have been published on the detection of additional ipsilateral lesions by breast ultrasonography or mammography. ${ }^{15}$ It also remains unclear whether whole breast sonography, rather than targeted ultrasonography, should be used in the diagnostic workup after recall. Frequently, additional ipsilateral lesions are diagnosed by pre-operative magnetic resonance imaging (MRI) in women with breast cancer. ${ }^{16}$

To our knowledge, data on additionally detected breast lesions following recall of a different mammographic abnormality are lacking. Therefore, the purpose of the current study was to assess the frequency, characteristics and outcome of additionally detected ipsilateral breast lesions after recall in women who attended a biennial screening mammography programme in the south of the Netherlands.

\section{Materials and methods}

\section{Study population}

This is a prospective observational follow-up study of women aged 50-75 years who attended a biennial breast cancer screening programme conducted in the south of the Netherlands. Details of the design of our breast cancer screening programme have been described previously. ${ }^{17,18}$ Women are personally invited by letter to attend the screening programme and the attendance rate is more than $80 \%$. Women being treated for breast cancer or those attending clinical follow-up after treatment of breast cancer do not attend the screening programme. Also, women with breast 
implants masking most of the fibroglandular tissue at mammography are advised to refrain from the programme. Otherwise, there are no exclusion criteria for screening. A consecutive series of 130,338 full-field digital mammography screens $(13,762$ initial screens and 116,576 subsequent screens) were included between January 1, 2014 and January 1, 2016. The screening mammograms were obtained at four specialised screening units (three mobile units and one fixed unit at Screening Program South).

Prior to participation, women are routinely asked for their permission to use their data for the evaluation of the screening programme and scientific purposes and all recalled women gave this permission. To minimise diagnostic bias, we excluded women who were recalled because of bilateral or multiple ipsilateral lesions, as additional diagnostic imaging procedures are more likely to be performed in these women than in women recalled for a unilateral lesion.

Ethical approval by our local Institutional Review Board was not required for this observational follow-up study, according to the Dutch Central Committee on Research involving Human Subjects (CCMO).The flow chart of screened women and subsequent inclusion is depicted in Figure 5.2.1.

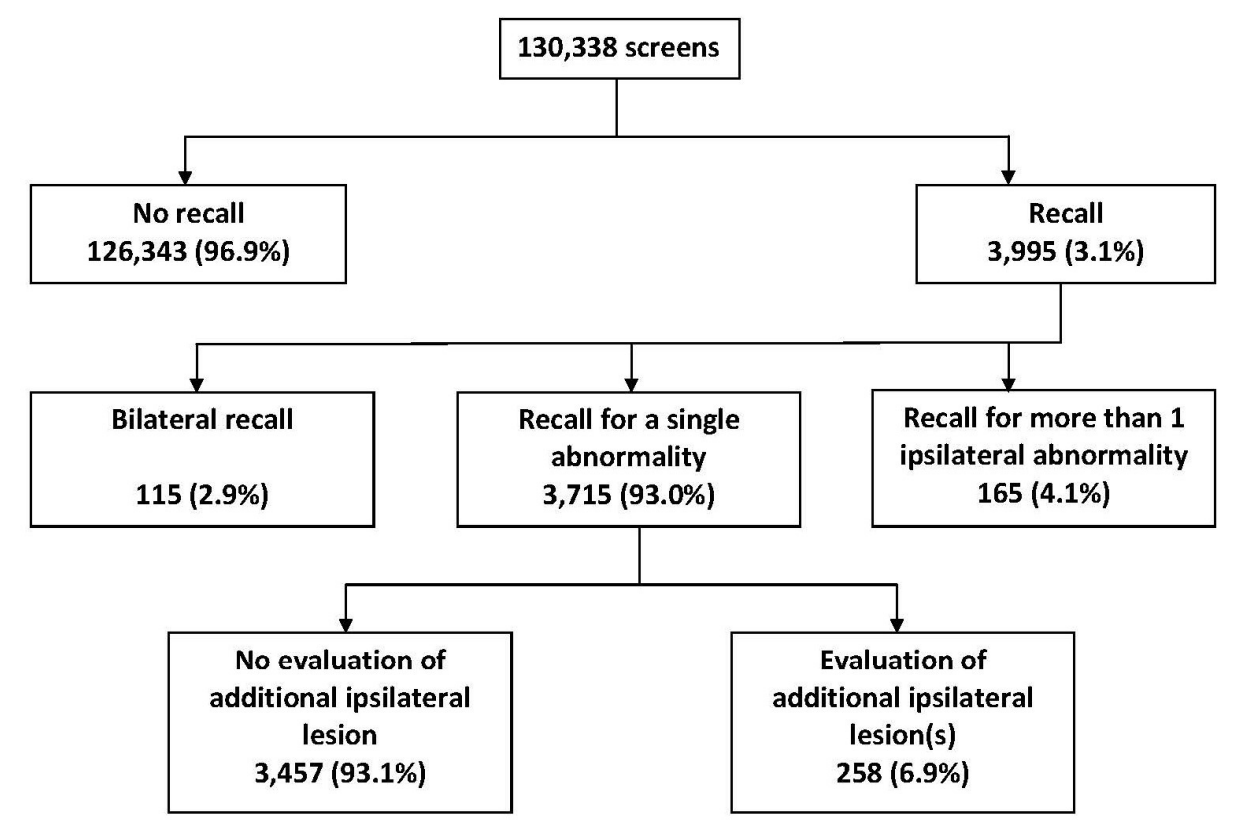

Figure 3.1.1 Flowchart of screened women and subsequent study inclusion.

\section{Screening procedure and recall}

All digital mammograms were obtained with a Lorad Selenia FFDM system (Hologiclnc, Danbury, CT), with a $70 \mathrm{~mm}$ pixel size and a $232 \times 286 \mathrm{~mm}$ field of view. The examinations were obtained by specialised screening mammography 
technologists and all screening mammograms were double read in a blinded fashion by a team of 12 certified screening radiologists. All but one of the screening radiologists read more than 10000 screens yearly in the screening region we currently report on. The remaining radiologist reads 3000 screens in this specific region and an additional 10000 screens in another Dutch screening region.

The screening radiologists first categorise abnormal mammographic findings into one of the following categories: suspicious mass, suspicious calcifications, suspicious mass combined with calcifications, architectural distortion, asymmetry or other abnormalities not otherwise categorized. Then, each screen is classified according to the Breast Imaging Reporting and Data System (BI-RADS) lexicon and the radiologists annotate each recalled mammographic abnormality on a drawing which is part of the recall report. ${ }^{19}$ Women with a BI-RADS 1 or 2 were not recalled. Women with a BIRADS 0,4 or 5 finding were referred to a dedicated breast unit of a hospital for further analysis. The BI-RADS category 3 is not used as short term follow-up is not available in the Dutch screening programme.

\section{Diagnostic work-up after recall}

Although a total of 20 hospitals were involved in the diagnostic work-up of recalled women, the diagnostic work up in the majority of women $(97.2 \%, 3883 / 3995)$ was performed in 7 regional hospitals in the south of the Netherlands. After physical examination by a surgical oncologist or dedicated breast nurse, additional breast imaging was performed at the radiology department. The screening mammogram was first reassessed by an experienced radiologist. The screening mammogram was routinely available and stored in the Picture Archiving and Communication System (PACS) of the hospital. Additional mammographic projections were obtained at the discretion of the radiologist. Full-field digital mammography was available in each of the 7 regional hospitals. Breast tomosynthesis was present in 2 hospitals from the beginning of the inclusion period and became available in 3 other hospitals in 2015. Breast ultrasonography was used for the additional characterisation of mammographic abnormalities and palpable breast lesions, for biopsy guidance and for target or second look purposes following breast MRI. Whole breast ultrasonography was not recommended, in accordance with the Dutch guidelines. ${ }^{20}$ Breast MRI was also available in each of the 7 hospitals and performed if indicated, as defined by the guidelines of the European Society of Breast Imaging ${ }^{21}$ and the Dutch guidelines. ${ }^{20}$ Fine-needle aspiration biopsy (FNAB), percutaneous core needle biopsy (CNB) and stereotactic biopsy were performed in each of the 7 hospitals, whereas MRI guided biopsy procedures were concentrated in the larger hospitals. All recalls were discussed by multidisciplinary teams that consisted of surgical oncologists, 
radiologists, medical oncologists, radiation oncologists, plastic surgeons, breast nurses and breast technologists.

During two-year follow-up, clinical data and data from diagnostic breast imaging, biopsy specimen and surgical procedures were collected of all recalled women by one of the screening radiologists (LD) and several radiology residents. The radiologist then entered all data in a database which had been constructed for quality assurance of the screening programme. Breast cancers were categorised into ductal carcinoma in situ (DCIS) and invasive cancers; lobular carcinoma in situ was considered a non-malignant lesion. The TNM (tumour, nodes and metastases) classification was used for malignant lesions. ${ }^{22}$ For all cancers treated by neoadjuvant therapy (either chemotherapy or anti-hormonal therapy), tumour size was derived from breast imaging (usually MRI) prior to the start of this therapy.

\section{Detection and assessment of ipsilateral breast lesions}

Any ipsilateral lesion for which a woman was not recalled (i.e., not annotated by the screening radiologists) and that was evaluated after recall by any means of additional imaging and/or biopsy was considered to be an additionally evaluated lesion. One of the screening radiologists (LD) recorded for each recall whether additional, nonrecalled ipsilateral lesions had been diagnosed following recall. The methods of detection and subsequent assessment of ipsilateral breast lesions for which the woman had not been recalled were derived from the clinical records and clinical radiology reports. Similar to the workup up of screen-detected mammographic abnormalities, biopsy data and surgical reports of ipsilateral breast lesions were obtained.

\section{Statistical analysis}

All relevant data of screened and recalled women are stored in a database which is used for quality assurance of the screening programme and for research purposes. For the current study, the study population was similar to the one we used to determine the frequency and outcome of contralateral breast abnormalities following recall. ${ }^{18}$ Descriptive statistics were performed using Statistical Package for Social Science 23.0 (SPSS Inc., IBM, Chicago, IL). The chi-square test was used to test for differences between women without and with additional ipsilateral breast lesions detected following recall. A p-value of less than 0.05 was considered to indicate a statistically significant difference. P-values were two sided.

There was no loss to follow-up. In a small number of cases, data was missing (e.g., estrogen or progesterone receptor status was missing due to an insufficient tissue 
sample). Because of the limited number of missing values, no statistical correction was performed. The chi-square test with post hoc Bonferroni-correction was used to compare detection performance of the imaging modalities.

\section{Results}

\section{Overall screening results}

Out of 130,338 consecutively screened women, 3995 (3.1\%) were recalled for further assessment. Breast cancer was diagnosed in 905 recalled women (including 163 (18.0\%) ductal carcinomas in-situ), resulting in an overall cancer detection rate of 6.9 per 1000 screens. The cancer detection rate was 8.6 per $1000(118 / 13,762)$ for initial screens and 6.8 per $1000(787 / 116,576)$ for subsequent screens. The overall positive predictive value (PPV) of recall was $22.7 \%$ (905/3995; $11.4 \%$ (118/1037) for initial screens and $26.6 \%$ (787/2958) for subsequent screens). Women who were recalled for a bilateral lesion $(n=115)$ and women with multiple recalled lesions in one breast $(n=165)$ were excluded from analysis (see also the flowchart, Figure 5.2.1). Patient characteristics were comparable for the remaining 3715 recalled women analysed in this study, with a trend towards denser breasts in women with evaluation of an additional ipsilateral lesion. Patient characteristics are presented in Table 5.2.1.

Table 5.2.1 Baseline characteristics of women without or with assessment of additional, non-recalled ipsilateral breast lesions.

\begin{tabular}{|c|c|c|c|c|}
\hline & $\begin{array}{l}\text { Overall } \\
\mathrm{N}=3,715\end{array}$ & $\begin{array}{c}\text { No additional } \\
\text { ipsilateral lesions } \\
\mathrm{N}=\mathbf{3 , 4 5 7}\end{array}$ & $\begin{array}{c}\text { Additional } \\
\text { ipsilateral lesions } \\
\mathbf{N}=\mathbf{2 5 8} \\
\end{array}$ & p-value* \\
\hline Median age, years & 58 & 58 & 57 & 0.89 \\
\hline \multicolumn{5}{|l|}{ Screening round, No (\%) } \\
\hline Initial & $944(25.4)$ & $887(25.4)$ & $57(22.0)$ & 0.22 \\
\hline Subsequent & $2771(74.6)$ & $2570(74.6)$ & $201(78.0)$ & \\
\hline \multicolumn{5}{|l|}{ Breast density, No (\%) } \\
\hline ACR I+II (0\%-50\%) & $2820(76,2)$ & $2637(76,2)$ & $183(71.0)$ & 0.06 \\
\hline ACR III+IV (50\%-100\%) & $895(23,8)$ & $820(23,8)$ & $75(29.0)$ & \\
\hline \multicolumn{5}{|c|}{ Previous breast surgery, No (\%) } \\
\hline Yes & $312(8,4)$ & $291(8,4)$ & $21(8.1)$ & 0.86 \\
\hline No & $3403(91.6)$ & $3166(91.6)$ & 237 (91.9) & \\
\hline \multicolumn{5}{|c|}{ Hormone replacement therapy, No (\%) } \\
\hline Yes & $159(4.3)$ & $150(4.3)$ & $9(3,5)$ & 0.50 \\
\hline No & $3556(95.7)$ & 3307 (95.7) & $249(96.5)$ & \\
\hline \multicolumn{5}{|c|}{ Family history of breast cancer, No (\%) } \\
\hline Yes & $494(13,5)$ & $465(13,5)$ & $29(11.2)$ & 0.30 \\
\hline No & 3221 (86.5) & $2992(86.5)$ & $229(88.8)$ & \\
\hline
\end{tabular}

$A C R=$ American College of Radiology. ${ }^{*}=$ women without assessment of additional ipsilateral lesions versus women with assessment of additional ipsilateral lesions 


\section{Detection of additional ipsilateral lesions}

A total of 258 women, $6.4 \%$ of all recalls, were analysed for an additional ipsilateral abnormality in the recalled breast.

In 182 women (70.5\%), the additional ipsilateral abnormality was first detected by review of the screening mammogram or additional mammographic views, with or without breast tomosynthesis. In 49 women (19.0\%), the lesions were found at ultrasound, whereas the remaining 27 lesions (10.5\%) were discovered at breast MRI. Breast MRI had been performed in 61 out of 258 women (23.6\%) with an additionally detected, ipsilateral breast abnormality. In 21 of these women (34.4\%), MRI was used for problem solving of an abnormality found at mammography or ultrasonography. In the remaining 40 women (65.6\%), MRI was performed in a pre-operative setting to exclude or confirm (unilateral or bilateral) multicentricity or multifocality of breast cancer or to determine tumour size. Figure 5.2.2 shows at which type of imaging modality the additional ipsilateral breast lesions were initially detected. The four imaging modalities showed no statistically significant differences in the number of lesions detected per imaging modality.

\section{Method of radiological detection of additional breast lesions in the recalled breast}

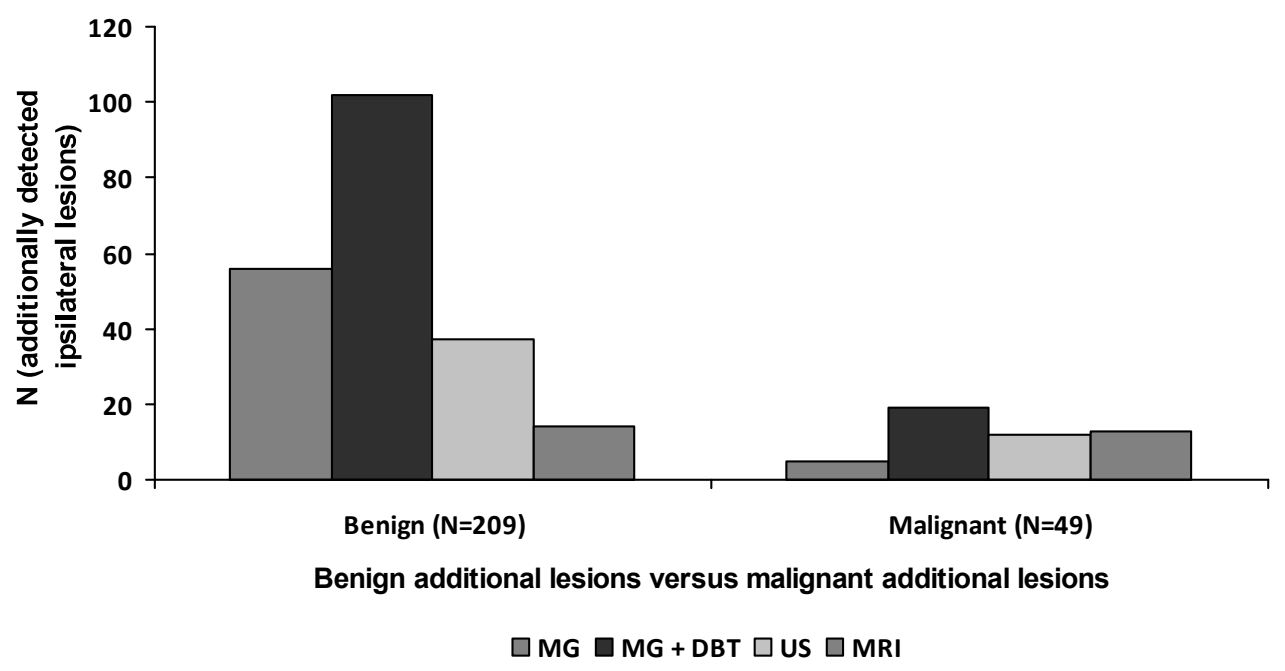

Figure 5.2.2 Detection of additional breast lesions in the recalled breast per imaging modality.

\section{Workup of additionally detected lesions in the recalled breast}

The diagnostic workup is summarized in Table 5.2.2. Biopsy was performed in $55.8 \%$ (144/258) of additionally detected ipsilateral lesions and in 39.7\% (1375/3457) of recalled lesions $(p<0.001$, Table 5.2 .3$)$. The majority of biopsies consisted of percutaneous CNB $(99 / 144,68.8 \%)$ and the positive predictive value of biopsy was 
significantly lower for additionally detected lesions than for recalled lesions $(34.2 \%$ (49/144) versus 54\% (743/1375), $(p<0.001)$.

Positive predictive value of biopsy for additionally detected lesions changed when separate analysis was performed in women with a proven malignant index lesion $(42 / 71,59.2 \%)$ versus women with a proven benign index lesion $(5 / 73,6.85 \%$, $\mathrm{p}<0.001)$.

Table 5.2.2 Outcome of recalled lesions versus additionally detected lesions in the recalled breast.

\begin{tabular}{lccc}
\hline & $\begin{array}{c}\text { Recalled lesions } \\
\mathbf{N = 3 , 4 5 7}\end{array}$ & $\begin{array}{c}\text { Additionally detected lesions } \\
\mathbf{N = 2 5 8}\end{array}$ & P-value \\
\hline Outcome, No (\%) & & & 0.34 \\
$\quad$ False positive (no cancer) & $2714(78.5)$ & $209(81)$ & \\
$\quad$ True positive (cancer) & $743(21.5)$ & & \\
Type of assessment after recall, No (\%) & & $114(44.2)$ & $<0.001$ \\
$\quad$ Clinical breast imaging & $2071(59.9)$ & $144(55.8)$ & $<0.001$ \\
$\quad$ Clinical breast imaging + biopsy & $1375(39.7)$ & 34.0 & \\
Positive predictive value of biopsy, \% & 54.0 & & \\
\hline
\end{tabular}

Table 5.2.3 Biopsy procedures and outcome of additionally detected lesions in the recalled breast/

\begin{tabular}{lcc}
\hline & \multicolumn{2}{c}{ Final outcome } \\
\cline { 2 - 3 } & Benign (209) & Malignant (49) \\
\hline Type of biopsy, No (\%) & $112(53.6)$ & $2(4.1)$ \\
No biopsy & & \\
Ultrasound guided biopsy & $13(6.2)$ & $2(4.1)$ \\
FNAB & $60(28.7)$ & $39(79.6)$ \\
CNB & $17(8.1)$ & $5(10.2)$ \\
SCNB & $1(0.5)$ & 0 \\
Percutaneous biopsy + excisional biopsy & $4(1.9)$ & $1(2.0)$ \\
Excisional biopsy & $2(1.0)$ & 0 \\
MRI biopsies N (\%) & & \\
\hline
\end{tabular}

FNAB = fine needle aspiration biopsy; CNB = core needle biopsy; SCNB = stereotactic core needle biopsy

\section{Additionally detected lesions in the recalled breast}

Of the 258 additionally detected ipsilateral lesions, 209 proved to be benign at biopsy or radiological follow-up. These lesions included cysts (81) and fibroadenomas (24), and to a lesser extent intramammary lymph nodes (15) or areas of fibrosis (10) and fibrocystic changes (4).

In 49 of the 258 women (19.0\%) with an additional lesion detected in the recalled breast, the lesion proved to be malignant. The characteristics of these malignancies are presented in Table 5.2.4 and compared to those of recalled women without analysis of additional lesions. The proportions of DCIS and invasive cancers were 
comparable for both groups, but additionally detected, ipsilateral malignancies more frequently showed a lobular histology $(28.5 \%$ versus $11.8 \%, p=0.001)$. Compared to screen-detected cancers, the screen-detected cancers with an additionally detected malignant lesion were more often stage T2 or higher $(p<0.001)$. In all but one of the 17 additionally detected cancers with lymph node involvement, the cancer was either part of a multifocal (10) or multicentric (6) malignancy. Lymph node involvement was more frequently present in the screen-detected cancers with an additionally detected cancer $(40.1 \%$ versus $20.9 \%, p=0.004)$. Mastectomy was more often performed in women diagnosed with an additional malignant ipsilateral lesion than in women with a unifocal, recalled malignancy $(55.1 \%$ versus $14.8 \%, p<0.001)$.

\section{Multifocal and multicentric malignancies}

Ipsilateral multifocal or multicentric breast cancer was present in 52 women who were recalled for the evaluation of a single abnormality at screening mammography. In 35 of the 49 (71\%) additionally detected malignancies, the histological features of these lesions were comparable to those of the index lesions and they were considered to be part of multifocal $(24 / 35,69 \%)$ or multicentric disease $(11 / 35,31 \%)$. Multifocality or multicentricity of breast cancer was histologically proven prior to surgery in 33 out of 35 lesions. The remaining 2 lesions were considered malignant on imaging alone and their malignant nature was confirmed at the surgical specimen.

The remaining 14 malignant lesions were either synchronous tumours $(n=9)$ or unifocal cancers in women whose recalled lesion turned out to be benign $(n=5)$. The synchronous cancers comprised six patients with DCIS, one invasive tubular cancer, one invasive ductolobular carcinoma and one invasive ductal carcinoma. These lesions showed a different histology than the ipsilateral, recalled malignancy. The histology of the five cancers diagnosed in women with a benign, recalled lesion in the ipsilateral breast was as follows: three invasive ductal carcinomas, one invasive lobular carcinoma and one DCIS. 
Table 5.2.4 Tumour characteristics of unilateral, screen-detected cancers versus additional cancers diagnosed in the ipsilateral recalled breast.

\begin{tabular}{|c|c|c|c|}
\hline & $\begin{array}{l}\text { Screen-detected } \\
\text { cancers } \\
n=743\end{array}$ & $\begin{array}{c}\text { Additionally detected } \\
\text { ipsilateral cancers } \\
n=49\end{array}$ & p-value \\
\hline Type of cancer, No (\%) & & & 0.44 \\
\hline Ductal carcinoma in situ (DCIS) & 139 (18.7) & $7(14.3)$ & \\
\hline Invasive & $604(81.3)$ & $42(85.7)$ & \\
\hline Histology of invasive cancers, No (\%) & & & 0.001 \\
\hline Ductal & $470(77.8)$ & $28(66.7)$ & \\
\hline Lobular & $71(11.8)$ & $12(28.5)$ & \\
\hline Ductolobular & $20(3.3)$ & $1(2.4)$ & \\
\hline Other & $43(7.1)$ & $1(2.4)$ & \\
\hline Tumour stage of invasive cancers, No (\%) & & & $<0.001$ \\
\hline $\mathrm{T} 1$ & $486(80.5)$ & $22(52.4)$ & \\
\hline $\mathrm{T} 2+$ & $118(19.5)$ & $20(47.6)$ & \\
\hline Lymph node status of invasive cancers, No (\%) & & & 0.004 \\
\hline $\mathrm{N}+$ & $126(20.9)$ & $17(40.1)$ & \\
\hline $\mathrm{N}-$ & 472 (78.1) & $25(59.9)$ & \\
\hline Unknown & $6(1.0)$ & 0 & \\
\hline Bloom \& Richardson grade, No (\%) & & & 0.01 \\
\hline 1 & 283 (46.9) & $12(28.5)$ & \\
\hline II & $252(41.7)$ & $25(59.5)$ & \\
\hline III & $68(11.3)$ & $5(12.0)$ & \\
\hline Unknown & $1(0.1)$ & 0 & \\
\hline Estrogen receptor status, No (\%) & & & 0.39 \\
\hline Positive & $546(90.3)$ & $36(85.8)$ & \\
\hline Negative & $55(9.2)$ & $6(14.2)$ & \\
\hline Unknown & $3(0.5)$ & 0 & \\
\hline Progesterone receptor status, No (\%) & & & 0.59 \\
\hline Positive & $427(70.7)$ & $32(76.2)$ & \\
\hline Negative & $175(29.0)$ & $10(23.8)$ & \\
\hline Unknown & $2(0.3)$ & 0 & \\
\hline Her2/Neu receptor status, No (\%) & & & 0.89 \\
\hline Positive & $61(10.1)$ & $4(9.5)$ & \\
\hline Negative & $539(89.2)$ & $38(90.5)$ & \\
\hline Unknown & $4(0.7)$ & 0 & \\
\hline Final surgical treatment, No (\%) & & & $<0.001$ \\
\hline Breast conserving surgery & 625 (84.1) & $22(44.9)$ & \\
\hline Mastectomy & $110(14.8)$ & $27(55.1)$ & \\
\hline No surgery & $8(1.1)$ & 0 & \\
\hline
\end{tabular}

\section{Discussion}

To our knowledge, this is the first study which focuses on the analysis of additionally detected breast lesions following recall of a different mammographic abnormality. We found that additional ipsilateral lesions were detected in $6.4 \%$ of recalled women. Type of assessment (clinical imaging versus clinical imaging + biopsy) was comparable 
for recalled lesions and additionally detected lesions, whereas the positive predictive value of biopsy was higher for recalled lesions. However, separate analysis showed that in the case of a malignant index lesion, the positive predictive value of biopsy for the additional lesion was higher compared to cases of benign index lesions $(p<0.001)$, which suggest that a low threshold for additional biopsy in proven malignant index lesions is warranted.

About $20 \%$ of the additionally detected lesions proved to be malignant and a majority of these malignancies were part of multifocal or multicentric disease. A majority of the additionally detected, malignant lesions were invasive ductal carcinomas. A recent metanalysis focusing on the detection of ipsilateral multifocal or multicentric cancer in women already diagnosed with breast cancer found comparable percentages of invasive cancers. ${ }^{23}$

In our series of 49 malignancies, additionally diagnosed in the recalled breast, 24 were histologically identical to the index lesion and located in the same breast quadrant (multifocal disease), whereas 11 malignancies with histological features identical to the index lesion were located in a different quadrant (multicentric disease). According to previous studies and a recent meta-analysis, multifocality may have a significant impact on 5 year survival rates, whereas the impact on disease free survival (DFS) is subject to debate, with a previous meta-analysis showing a trend towards a poorer DFS (HR 1.96; $p=0.07) .{ }^{23-26}$

The majority of additionally detected lesions in recalled breasts showed a benign outcome and they were mostly cysts and fibroadenomas. Recent studies show a comparable rate of additionally found benign lesions in recalled women, although a larger proportion of fibroadenomas were found at contrast enhanced mammography. ${ }^{15,27,28}$ In a majority of women, analysis by imaging alone was sufficient to confirm the benign nature of additionally detected lesions. Surgical excision of benign breast lesions should be kept to a minimum. Excisional biopsy may cause changes in the breast tissue, impairing a proper assessment of future screening mammograms. The latter may result in an increased false positive recall rate and a lower sensitivity of screening mammography. ${ }^{29}$

Almost half of the additional ipsilateral lesions were detected on digital mammography combined with breast tomosynthesis. With the increased use of tomosynthesis in a diagnostic setting, it can be expected that more additional lesions will be detected after recall. Tomosynthesis has not yet been implemented in the Dutch mammography screening programme. Several studies have shown that breast tomosynthesis in a screening setting, in addition to full field digital mammography, increases the cancer detection and may decrease false positive recall rates. ${ }^{6,7}$ On the 
other hand, an Italian screening study reported a higher false-positive recall rate when adding tomosynthesis to digital mammography. ${ }^{5}$ and a recent Swedish screening study also found a higher false positive recall rate for one view tomosynthesis compared to digital mammography. ${ }^{4}$ Higher recall rates and higher false positive rates may likely decrease again when radiologists gain more experience with tomosynthesis in a screening setting. ${ }^{4-7}$ Certain types of breast cancer, especially invasive lobular carcinoma, can be difficult to detect as they may present as subtle architectural distortions which are more likely to be missed at screening mammography. At screening, tomosynthesis may be helpful to detect these distortions. In the diagnostic setting, it can be of value to find additional multicentric or multifocal lesions and guide ultrasound imaging when the index lesion presents with an architectural distortion. Vigilance is required and one should not be mislead by the satisfaction of having found one breast abnormality as additional malignant lesions may be present elsewhere in the breast. ${ }^{8-11,24,30}$

In our study, a majority of the additionally detected malignant lesions (64\%) were found in the same quadrant as the index lesion and a considerable number of these malignant lesions (30\%) were detected first at ultrasound. Breast ultrasonography of the quadrant harbouring the cancer is routinely performed, in adherence to the guidelines of the European Society of Breast Imaging ${ }^{21}$ and the Dutch guidelines ${ }^{20}$, whereas whole breast ultrasonography is not recommended as it may introduce many more increased false positive findings than the detection of additional cancers. Another substantial number of additional ipsilateral malignancies were found at breast MRI. According to the Dutch guidelines, indications for breast MRI include the evaluation of multicentric, multifocal or contralateral disease in case of invasive lobular cancer, and determination of tumour size in dense breasts. In select cases, MRI can be employed for problem solving of indeterminate lesions at mammography and/or ultrasonography. ${ }^{23}$ European guidelines and Dutch guidelines recommend preoperative MRI imaging for most cases of invasive lobular carcinomas and they do not recommend a routine use of breast MRI for invasive ductal carcinoma or ductal carcinoma in situ. The higher proportion of invasive lobular cancer among additionally detected ipsilateral cancers may probably be explained by the more intensified use of $\mathrm{MRI}$ in invasive lobular cancer than invasive ductal cancer.

Synchronous malignancy was found in nine women with assessment of an additional ipsilateral lesion. The majority of these lesions were DCIS, ranging from low-grade to high grade DCIS. Diagnosis and treatment of DCIS has been studied extensively, also with respect to the issue of overdiagnosis and overtreatment of early breast cancer. Consensus has yet to be reached on this topic. Research on the treatment of synchronous DCIS is sparse, but it will probably have a significant impact on the proposed treatment regimen. The treatment not only concerns surgical 
planning, but it also comprises decisions on radiation therapy. The woman's preference for mastectomy or breast conserving surgery should also be taken in account as this decision may as well be influenced by the presence of synchronous malignant lesions. ${ }^{31,32}$ In this study, women with an additional malignancy diagnosed in the breast already harbouring a recalled breast cancer were much more likely to undergo mastectomy than women with only a malignant recalled lesion (55.1\% versus $14.8 \%, P<0.001)$. In these cases multifocality and multicentricity may warrant extensive surgery, without the possibility of breast conserving surgery.

An ipsilateral breast cancer was diagnosed in five women whose recalled mammographic abnormality proved benign. The lesions were either located in the same quadrant as the recalled lesion and detected at ultrasound $(2 / 5)$, or located in a different quadrant and detected by mammography with (2/5) or without tomosynthesis (1/5). We do not know whether the lesions, located in different quadrants, were visible at screening mammography, but previous research suggests that additional cancers may be missed at screening as a result of satisfaction of search.

Our study has certain limitations. Our results may not be representative for other regional or nationwide screening mammography programmes. Programmes may show variations in reading strategies (single reading versus double reading), screening interval (1-3 years), radiological equipment, and differences in recall rates. Also, a non-uniform use of imaging modalities after recall may have influenced study outcome. The moderate sample size of ipsilateral cancers may limit the external validation of the results.

Breast imaging techniques are continuously updated and new techniques are emerging and being implemented, which may increase the number of abnormalities detected at screening and the number of additional lesions in recalled women. Although one should aim for an increased cancer detection, this must not be accompanied by a significant increase in false positive findings that may introduce unnecessary diagnostic investigations, unnecessary treatment, and avoidable psychological impact.

In summary, additionally detected ipsilateral breast lesions are usually benign. The majority of malignant lesions are part of a multifocal or multicentric screen detected malignancy. Synchronous tumours comprise a minority of the additionally detected malignant lesions. Additional radiological imaging and biopsy is frequently required to determine the nature of lesions additionally detected in recalled breasts. 


\section{References}

1. Gotzsche PC, Jorgensen KJ. Screening for breast cancer with mammography. Cochrane Database Syst Rev 2013, CD001877.

2. Sankatsing VDV, van Ravesteyn NT, Heijnsdijk EAM, Looman CWN, van Luijt PA, Fracheboud J, den Heeten GJ, Broeders MJM, de Koning HJ. The effect of population-based mammography screening in Dutch municipalities on breast cancer mortality: 20 years of follow-up. Int J Canc 2017;141:671-677.

3. Otto SJ, Fracheboud J, Looman CW, Broeders MJ, Boer R, Hendriks JH, Verbeek AL, de Koning HJ. National Evaluation Team for Breast Cancer S. Initiation of population-based mammography screening in Dutch municipalities and effect on breast-cancer mortality: a systematic review. Lancet 2003;361:1411-1417.

4. Lang K, Andersson I, Rosso A, Tingberg A, Timberg P, Zackrisson S. Performance of one-view breast tomosynthesis as a stand-alone breast cancer screening modality: results from the Malmo Breast Tomosynthesis Screening Trial, a population-based study. Eur Radiol 2016;26:184-190.

5. Bernardi D, Macaskill P, Pellegrini M, Valentini M, Fanto C, Ostillio L, Tuttobene P, Luparia A, Houssami N. Breast cancer screening with tomosynthesis (3D mammography) with acquired or synthetic 2D mammography compared with 2D mammography alone (STORM-2): a population-based prospective study. Lancet Oncol 2016;17:1105-1113.

6. Skaane P, Bandos AI, Gullien R, Eben EB, Ekseth U, Haakenaasen U, Izadi M, Jebsen IN, Jahr G, Krager M, Niklason LT, Hofvind $S$, et al. Comparison of digital mammography alone and digital mammography plus tomosynthesis in a population-based screening program. Radiology 2013;267: 47-56.

7. Ciatto S, Houssami N, Bernardi D, Caumo F, Pellegrini M, Brunelli S, Tuttobene P, Bricolo P, Fanto C, Valentini M, Montemezzi S, Macaskill P. Integration of 3D digital mammography with tomosynthesis for population breast-cancer screening (STORM): a prospective comparison study. Lancet Oncol 2013;14:583-589.

8. McDonald ES, Oustimov A, Weinstein SP, Synnestvedt MB, Schnall M, Conant EF. Effectiveness of digital breast tomosynthesis compared with digital mammography: outcomes analysis from 3 years of breast cancer screening. JAMA Oncol 2016;2:737-743.

9. Maxwell AJ, Michell M, Lim YY, Astley SM, Wilson M, Hurley E, Evans DG, Howell A, Iqbal A, Kotre J, Duffy S, Morris J. A randomised trial of screening with digital breast tomosynthesis plus conventional digital 2D mammography versus 2D mammography alone in younger higher risk women. Eur J Radiol 2017;94:133-139.

10. Friedewald SM, Rafferty EA, Rose SL, Durand MA, Plecha DM, Greenberg JS, Hayes MK, Copit DS, Carlson KL, Cink TM, Barke LD, Greer LN, et al. Breast cancer screening using tomosynthesis in combination with digital mammography. J Am Med Assoc 2014;311:2499-2507.

11. McCarthy AM, Kontos D, Synnestvedt M, Tan KS, Heitjan DF, Schnall M, Conant EF. Screening outcomes following implementation of digital breast tomosynthesis in a general-population screening program. J Natl Cancer Inst 2014;106.

12. El Sharouni MA, Postma EL, Menezes GL, van den Bosch MA, Pijnappel RM, Witkamp AJ, van der Pol CC, Verkooijen HM, van Diest PJ. High prevalence of MRI-detected contralateral and ipsilateral malignant findings in patients with invasive ductolobular breast cancer: impact on surgical management. Clin Breast Canc 2016;16:269-275.

13. Gutierrez. High cancer yield and positive predictive value: outcomes at a center routinely using preoperative breast MRI for staging. AJR Am J Roentgenol 2011;196:93-99.

14. Sardanelli. Overview of the role of pre-operative breast MRI in the absence of evidence on patient outcomes. Breast 2010;19:3-6. 
15. He H, Plaxco JS, Wei W, Huo L, Candelaria RP, Kuerer HM, Yang WT. Incremental cancer detection using breast ultrasonography versus breast magnetic resonance imaging in the evaluation of newly diagnosed breast cancer patients. Br J Radiol 2016;89:20160401.

16. Plana MN, Carreira C, Muriel A, Chiva M, Abraira V, Emparanza Jl, Bonfill X, Zamora J. Magnetic resonance imaging in the preoperative assessment of patients with primary breast cancer: systematic review of diagnostic accuracy and meta-analysis. Eur Radiol 2012;22:26-38.

17. Duijm LE, Groenewoud JH, Jansen FH, Fracheboud J, van Beek M, de Koning HJ. Mammography screening in The Netherlands: delay in the diagnosis of breast cancer after breast cancer screening. $\mathrm{Br}$ J Canc 2004;91:1795-1799.

18. Lameijer JRC, Coolen AM, Voogd AC, Strobbe LJ, Louwman MWJ, Venderink D, Tjan-Heijnen VC, Duijm LEM. Frequency and characteristics of contralateral breast abnormalities following recall at screening mammography. Eur Radiol 2018;28(10):4205-4214.

19. American College of Radiology. BI-RADS Committee. ACR BI-RADS atlas: breast imaging reporting and data system. fifth ed. Reston, VA: American College of Radiology; 2013.

20. NABON. Richtlijn Mammacarcinoom, vol. 2017; 2012.

21. Mann RM, Balleyguier C, Baltzer PA, Bick U, Colin C, Cornford E, Evans A, Fallenberg E, Forrai G, Fuchsjager $\mathrm{MH}$, Gilbert FJ, Helbich $\mathrm{TH}$, et al. Breast MRI: EUSOBI recommendations for women's information. Eur Radiol 2015;25:3669-3678.

22. Sobin LH, Gospodarowicz MK, Wittekind C. International Union against Cancer. TNM classification of malignant tumours. seventh ed. Chichester, West Sussex, UK ; Hoboken, NJ: Wiley-Blackwell; 2010. p. 310. $x x$.

23. Kanumuri $P$, Hayse B, Killelea BK, Chagpar AB, Horowitz NR, Lannin DR. Characteristics of multifocal and multicentric breast cancers. Ann Surg Oncol 2015;22:2475-2482.

24. Vera-Badillo FE, Napoleone M, Ocana A, Templeton AJ, Seruga B, Al-Mubarak M, AlHashem H, Tannock IF, Amir E. Effect of multifocality and multicentricity on outcome in early stage breast cancer: a systematic review and meta-analysis. Breast Canc Res Treat 2014;146:235-244.

25. Weissenbacher TM, Zschage M, Janni W, Jeschke U, Dimpfl T, Mayr D, Rack B, Schindlbeck C, Friese K, Dian D. Multicentric and multifocal versus unifocal breast cancer: is the tumor-node-metastasis classification justified? Breast Canc Res Treat 2010;122:27-34.

26. Houssami N, Ciatto S, Macaskill P, Lord SJ, Warren RM, Dixon JM, Irwig L. Accuracy and surgical impact of magnetic resonance imaging in breast cancer staging: systematic review and meta-analysis in detection of multifocal and multicentric cancer. J Clin Oncol 2008;26:3248-3258.

27. Houben IPL, Van de Voorde P, Jeukens C, Wildberger JE, Kooreman LF, Smidt ML, Lobbes MBI. Contrast-enhanced spectral mammography as workup tool in patients recalled from breast cancer screening has low risks and might hold clinical benefits. Eur J Radiol 2017;94:31-37.

28. Lobbes MB, Lalji U, Houwers J, Nijssen EC, Nelemans PJ, van Roozendaal L, Smidt ML, Heuts E, Wildberger JE. Contrast-enhanced spectral mammography in patients referred from the breast cancer screening programme. Eur Radiol 2014;24:1668-1676.

29. van Breest Smallenburg V, Duijm LE, Voogd AC, Groenewoud JH, Jansen FH, van Beek M, Louwman MW. Lower sensitivity of screening mammography after previous benign breast surgery. Int J Canc 2012;130:122-128.

30. Bahl M, Gaffney S, McCarthy AM, Lowry KP, Dang PA, Lehman CD. Breast cancer characteristics associated with 2D Digital Mammography versus Digital Breast Tomosynthesis for screening-detected and interval cancers. Radiology 2017, 171148.

31. Groen EJ, Elshof LE, Visser LL, Rutgers EJT, Winter-Warnars HAO, Lips EH, Wesseling J. Finding the balance between over- and under-treatment of ductal carcinoma in situ (DCIS). Breast 2017;31: 274-283.

32. Iacconi C, Galman L, Zheng J, Sacchini V, Sutton EJ, Dershaw D, Morris EA. Multicentric cancer detected at breast MR imaging and not at mammography: important or not? Radiology 2016;279:378- 384. 


\section{Chapter 6}

General discussion and future perspectives 



\section{General discussion and future perspectives}

The goal of population-based breast cancer screening is to reduce breast cancer mortality and morbidity by detecting breast cancer at an early stage without substantially increasing the number of false positive findings. The balance between recall rate, cancer-detection rate $(C D R)$ and false positive rate is a delicate one. The importance of maintaining low false-positive rates is threefold. Women who experience a false positive recall show a higher prevalence of long-term psychosocial consequences compared with those who had a negative screen. ${ }^{1}$ Although these consequences decrease over time, they remain consistently higher compared with controls. ${ }^{1,2}$ In line with these results, a study performed in a southern region of the Dutch breast cancer screening programme, showed that women with a false positive screen had a low quality of life (QoL) and feelings of anxiety, lasting for at least 1 year. ${ }^{3} \mathrm{~A}$ false-positive recall also results in higher overall screening costs because of additional imaging and biopsy procedures. ${ }^{4}$ And finally, false positive screens result in a significantly lower re-attendance rate, possibly lowering the overall effectiveness and cost-effectiveness of the screening programme. ${ }^{5}$

\section{Radiologist blinded double reading}

In conjunction with European guidelines ${ }^{6}$, double reading has been standard of care in the Dutch nationwide breast cancer screening programme for many years now. Cancer detection rates have been shown to increase with non-blinded double reading compared to single reading. However, these studies mostly date from the screen-film mammography (SFM) era and employ non-blinded double reading. Data on the added value of blinded double reading compared to single reading is sparse. When the introduction of full-field digital mammography (FFDM) in our screening region was completed in 2010, blinded double reading became technically possible. A previous study in our screening region comparing independent (non-blinded) and blinded double reading has shown that blinded double reading increases cancer detection rate and programme sensitivity, at the expense of an increase in recall rate and false positive recall rates. $^{7}$

\section{Impact of the second reader at blinded double reading}

In chapter $\mathbf{2}^{8}$ we determined the impact of the second reader on screening outcome at blinded double reading of digital screening mammograms. We found that the cancer detection rate significantly increases (from 6.2 to 7.0 per 1000 screens; $\mathrm{p}<0.001)$ with the addition of a second reader, compared to single reading. This increase in CDR comes at the expense of a significant increase in recall rate (3.0 to $3.6 \% ; p<0.001)$ and false positive recall rate $(23.4$ to 28.7 per 1000 screens; $p<0.001$ ). 
In two previous studies, Posso et. al. found a comparable CDR of 4.2-4.8 and 4.65.2 per 1000 screens at single reading and blinded double reading, respectively. ${ }^{4,9}$ Like in our study, the recall rate significantly increased with blinded double reading. Our increase in recall rate is at least partly explained by the fact that all discordant readings were recalled without consensus or arbitration, whereas Posso et. al performed consensus with arbitration of discordant recalls. Arbitration of discordant recalls has been shown to reduce recall rates. ${ }^{10,11}$ In contrast to the studies by Posso et. al., our recall rate is still well within the ranges of the European quality assurance guidelines as well as the Dutch optimisation study. ${ }^{6,12}$ Furthermore, our screening programme is characterised by a much higher CDR and PPV of recall.

Because of the lack of an increase in CDR in their study, it is not surprising Posso et. al. concluded that blinded double reading is not a cost-effective reading strategy. In our study, we did find a significant increase in CDR for blinded double reading compared with single reading. Even though we did not perform a cost-effectiveness analysis, since only a small portion (less than 10\%) of total screening examination costs ( $€ 66$ in 2014) are spent on the screening radiologists, a change from blinded double reading to single reading is also not likely to result in a significant cost reduction. Therefore, we favour blinded double reading over single reading and blinded double reading is still standard of care in our screening programme.

The majority of screen-detected cancers additionally detected by the second reader were small (T1) invasive cancers $(64.6 \%)$, mostly of low histological grade, or DCIS (25.6\%), mostly (76.2\%) intermediate and high grade. These findings suggest that blinded double reading detects additional cancers at an early stage, likely reducing breast cancer mortality and morbidity. However, they might also contribute to overdiagnosis and overtreatment. Recent studies suggest that some of these small invasive cancers with favourable biological behaviour do not progress to clinically significant cancers during the patient's lifetime. ${ }^{13,14}$ In our study, additionally detected cancers comprise mostly T1, low grade invasive cancers. However, a substantial proportion (41.0\%) of these invasive cancers additionally detected by the second reader were grade II and III invasive cancers. Moreover, $13.1 \%$ of additionally detected tumours were larger than $20 \mathrm{~mm}(\mathrm{~T} 2+), 18.0 \%$ were lymph node positive cancers and $33.3 \%$ of DCIS were high grade. These additionally detected cancers therefore likely represent a mix of early detection of clinically relevant breast cancers and overdiagnosis.

\section{Frequency and characteristics of discordant recalls}

We found that the proportion of BI-RADS 0 recalls was higher in the group of women additionally recalled by the second reader. The BI-RADS 0 category represents 
abnormalities with a low suspicion for malignancy and thus a high percentage of false positives. ${ }^{11}$ Because of our study design, additional recalls by the second reader are by definition discordant recalls. However, there are likely also discordant readings in the group of women recalled by the first reader. Because two radiologists are more likely to agree on a more obvious abnormality (i.e., BI-RADS 4 or 5 lesion), it is likely that in this group of discordant first reader recalls, the percentage of BI-RADS 0 abnormalities is also high. In order to further optimise blinded double reading of screening mammograms, it is important to gain insight into what causes a discordant recall. In chapter $3^{15}$ we therefore compared mammographic and tumour characteristics of screen-detected cancers following concordant or discordant recalls.

More than a quarter of recalls at blinded double reading was based on a discordant reading. Screen-detected cancers detected through a discordant reading tended to show a more favourable tumour grading with a trend towards smaller tumours, less frequently associated with lymph node metastasis. Proportions of invasive cancers and DCIS were comparable for concordant and discordant screendetected cancers. In both groups most cancers were small T1 invasive cancers, which were mostly hormone receptor positive. Also, the percentage of low-grade DCIS, a potential candidate for overdiagnosis ${ }^{16}$, did not differ between both groups. It is therefore not likely that discordant readings contribute to a substantial increase in overdiagnosis.

Results from this study are in line with our expectations from chapter $2^{8}$ : the majority of discordant recalls is based on a BI-RADS 0 abnormality and the proportion of BI-RADS 0 recalls is significantly higher compared to the group of concordant recalls (75.7\% vs. $56.3 \%, \mathrm{p}<0.001)$. As discussed previously, this is most likely due to the fact that two readers are more likely to agree on a more obvious (BI-RADS 4 or 5) abnormality. This hypothesis is supported by the finding that the percentage of 'subtle' abnormalities (i.e., asymmetries and, to a lesser extent, architectural distortions) was indeed higher in group of discordant readings. The percentage of microcalcifications and suspicious masses was higher in the concordant reading group. Inter-observer variability in using the BI-RADS lexicon ${ }^{17}$ is also more likely to have an impact on screening outcome in the setting of blinded double reading than at nonblinded double reading. The aforementioned differences in mammographic characteristics provide possible strategies for improvement, for example in training screening radiologists. Additional emphasis on the detection and classification of asymmetries and architectural distortions might be beneficial. There might also be a role for digital breast tomosynthesis (DBT). For example, Durand et. al showed that DBT lowers recall rate, most often for asymmetries. ${ }^{18}$ Dibble et. al. showed that DBT decreases inter-observer variability and increases reader confidence in detecting architectural distortions. ${ }^{19}$ 


\section{Value of technologist's reading and quality assurance session}

Previous studies have investigated the role of screening technologists in mammography reading. In our screening programme, technologists do not function as official readers, but nonetheless have always been encouraged to look for mammographic abnormalities. Their training therefore not only focusses on mammography technique and positioning, but also on breast anatomy and pathology. They obtain and review a mammogram and annotate any abnormality for which they deem recall necessary.

\section{Arbitration of discordant radiologist readings}

As discussed earlier, radiologist blinded double reading generates many discordant readings. Many of these comprise low suspicion BI-RADS 0 abnormalities, unfortunately increasing recall rate and false positive recall rate. Several strategies on how to deal with these discordant recalls have been studied. For example, Klompenhouwer et. al studied third reader arbitration of all discordant readings ${ }^{10}$ and discordant BI-RADS 0 readings only ${ }^{11}$ and prefer the latter strategy as it increases recall rates and PPV of recall without lowering CDR and programme sensitivity. Ciatto et. al and Caumo et. al also studied arbitration of discordant readings whereas Shaw et. al studied consensus review. ${ }^{20-22}$ No consensus has been reached as to which strategy yields optimal results. Studies by Duijm et. al. ${ }^{23}$ and Torres-Mejia et. al. ${ }^{24}$ have shown that trained screening mammography technologists may detect breast cancers that are missed by screening radiologists. The role of technologists in consensus and arbitration of discordant recalls has not previously been investigated. In chapter $4.1^{25}$ we therefore determined whether technologist readings of screening mammograms could be used to decide which discordant radiologist readings should be recalled.

We found that $28.0 \%(998 / 3562)$ of recalls was based on a discordant reading between two radiologists, mostly (76.1\%) based on a BI-RADS 0 abnormality. These findings are in line with our results from chapter 2 and 3. Technologists would have recalled $33.8 \%$ (337/998) of these women of which $11.9 \%$ (40/337) were diagnosed with breast cancer. In the group of women who would not have been recalled by the technologists, 9.1\% (60/661) were diagnosed with breast cancer. These were mostly invasive cancers, of which 17 (36.2\%) were larger than $10 \mathrm{~mm}$ in size, 19 (40.4\%) were grade II or III and $8(17.0 \%)$ showed lymph node metastasis.

Using the technologists reading to decide which discordant readings should be recalled, decreases recall rate and increases PPV of recall at the expense of a borderline significant decrease in cancer detection rate (from 7.1/1000 screens to $6.5 / 1000$ screens, $p=0.10$ ). The proportion of detected breast cancers was comparable for discordant readings with or without a positive technologist reading, irrespective of 
recall BI-RADS. This finding indicates that the technologist's reading provides no substantial discriminating ability. This is, at least partly, in contrast with results from previous studies indicating a comparable sensitivity of radiologists and technologists in reading screening mammograms at the expense of an increase FPR. ${ }^{23,24,26-29}$ This is largely explained by a difference in mammography technique (FFDM vs. SFM) and reading strategy (blinded double reading vs. single reading or independent double reading). Another possible explanation for the limited benefit of the current strategy is the difference between radiologists and technologists in mammography reading experience and the amount of feedback received. The lack of discriminating ability combined with the decrease in cancer detection rate, led to our conclusion that there appears to be no added value of using the technologists' reading to decide which discordant readings should be recalled.

\section{Value of quality assurance sessions in additional breast cancer detection}

In the Dutch nationwide breast cancer screening programme, technologists attend 6-weekly quality assurance sessions under supervision of a coordinating screening radiologist. These sessions serve multiple purposes, mainly quality assurance and continued training. At these sessions, all mammograms that were considered suspicious by the technologists but not by the screening radiologists are discussed. A woman is recalled at this stage, if the coordinating screening radiologists considers recall necessary. We know that additional breast cancers are detected through these sessions but data on numbers and characteristics are lacking. In chapter $4.2^{30}$ we determined the frequency and characteristics of these cancers.

During our 8-year inclusion period, 14,142 women were recalled, resulting in 3156 screen-detected cancers. Technologists would have recalled 2320 of these, meaning that they would have missed $830(26 \%, 830 / 3156)$ breast cancers. However, since the radiologists were not blinded to the technologists reading and reading circumstances differed, we cannot compare reading performance. All 11,627 technologist positive mammograms classified by the radiologists as BI-RADS 1 or 2 (i.e., no recall), were discussed during quality assurance sessions. This resulted in 85 additional recalls and 26 screen-detected cancers $(0.8 \%$ of all screen-detected cancers) resulting in a sensitivity for additional cancer detection of 52\% (26/50). Most of these cancers were invasive and larger than $10 \mathrm{~mm}$ in size and included six lymph node positive tumours and three triple negative tumours. Therefore, these additionally detected cancers probably do not reflect overdiagnosis. When comparing the 26 additionally detected cancers to the 3130 cancers detected through radiologist double reading, we found no significant differences in mammographic and tumour characteristics. 
In the group of women with either a screen-detected cancer at radiologist double reading or an interval cancer there were 104 and 24 women, respectively, with a technologist positive screening for the same abnormality in a previous screening round. These 128 cancers could be considered as 'missed' at quality assurance sessions at the time of the technologist positive screening. We compared them with the 26 cancers that were detected through these sessions and again found no significant differences in mammographic characteristics. However, there was a trend towards more invasive cancers in the group of 'missed' cancers and a trend towards more triple-negative tumours in the group of cancers detected through quality assurance.

Previous studies have shown that in single reading, additional technologist review is helpful in selecting cases for blinded double reading by a second radiologist and that secondary recall of technologist positive screenings was a successful strategy for increasing CDR. ${ }^{23,29}$ In this study, only $3.4 \%$ of women with interval cancers had a technologist positive screening for the same abnormality in a previous screening round. Also, only $0.2 \%$ of women who were discussed at quality assurance sessions were diagnosed with breast cancer. These combined findings suggest that technologist readings provide no substantial discriminating ability in deciding which negative screenings at double reading are eligible for secondary recall. This is in contrast with results from aforementioned previous studies but in line with results from chapter $4.1 .^{25}$ The role of quality assurance sessions in additional cancer detection is therefore limited.

\section{Frequency and characteristics of additional breast abnormalities following screening mammography}

A side effect to population based breast cancer screening is that additional, nonrecalled, breast abnormalities may be detected in the contralateral and/or ipsilateral breast at clinical work-up after recall. Whether or not this is an undesirable side effect depends on number and type of lesions detected, or rather if these abnormalities are clinically relevant. Therefore, in chapter $5.1^{31}$ and chapter $5.2^{32}$, we studied the frequency and characteristics of additionally detected breast lesions in the contralateral and ipsilateral breast, respectively.

We found that additional ipsilateral breast abnormalities were found in $6.4 \%$ of recalled women. In $3.2 \%$ of recalled women, an additional contralateral breast abnormality was found. Most additionally detected lesions were benign (comprising mostly cysts and fibroadenomas) and were detected through mammography, either by reviewing the screening mammogram or on additional mammographic views, with or without digital breast tomosynthesis (DBT). In $42.6 \%$ of ipsilateral and in $55.8 \%$ of 
contralateral lesions, biopsy was performed. This resulted in a PPV of biopsy of $36.4 \%$ and $34.0 \%$, respectively. In both cases this is significantly lower than PPV of biopsy of recalled lesions (54.0\% and $53.1 \%$, respectively).

Contralateral additional abnormalities were malignant in $15.5 \%$ of cases. In retrospect, $20.0 \%$ of these additional contralateral malignancies were considered to be missed at the latest screening examination. This is in line with a previously reported sensitivity of screening mammography for detection of bilateral breast cancer of less than $20 \% .{ }^{33}$ Additionally detected ipsilateral abnormalities proved to be malignant in $19.0 \%$ of cases and most of these cancers were part of a multifocal or multicentric screen-detected cancer. This is in line with results from a metanalysis on the detection of ipsilateral multicentric or multifocal breast cancers in women already diagnosed with breast cancer ${ }^{34}$, reporting similar percentages of invasive cancers. In case of a malignant recalled lesion, PPV of biopsy of additional ipsilateral lesions was higher when compared with a benign recalled lesion. This implicates that a low biopsy threshold for additional abnormalities is warranted in case of a malignant index lesion. Tumour characteristics were comparable for screen-detected cancers and additionally detected malignancies, both in the ipsilateral and contralateral breast. Most breast cancers were T1 invasive ductal cancers, mostly grade I or II, without lymph node involvement. For contralateral breast cancers, this is in contrast with results from a previous Dutch study in which the authors found more lobular cancers and less lymph node positive cancers in the group of contralateral synchronous breast cancers compared with index cancers and unilateral cancers. ${ }^{33}$

As breast imaging techniques improve and new imaging techniques are being implemented in both clinical and screening setting, it is likely that the number of additionally detected abnormalities after recall will increase. For example, almost half of the additionally detected ipsilateral lesions in our study were detected on full-field digital mammography combined with digital breast tomosynthesis (DBT). Previous studies on DBT in breast cancer screening have shown that DBT increases cancer detection rates. ${ }^{35-37}$ However, in some studies, this was accompanied by an increase in false positive rates. ${ }^{38,39}$ In the Trento DBT pilot study, DBT improved early detection but not interval cancer rates, suggesting that it could add benefit as well as over detection in population-based breast cancer screening. ${ }^{35}$ DBT is increasingly being used in a diagnostic setting but has not yet been implemented in the Dutch screening programme.

Studies have shown an improved cancer detection when MRI or whole-breast ultrasound is added to mammography. ${ }^{40-42}$ This, however, is accompanied by an increase in false positive findings, an undesirable side-effect. Therefore, the standard use of whole-breast ultrasound or MRI with proven breast cancer is not 
recommended in both European and Dutch guidelines. ${ }^{43,44}$ Recently, the Dutch DENSE trial has shown that the use of supplemental MRI screening in women with extremely dense breast tissue and a normal initial screening mammogram results in significantly lower interval cancers. ${ }^{45}$ In our study, recalled women who were evaluated for an additional abnormality more often had dense breast tissue compared with women who were not evaluated for additional abnormalities. This is likely due to the fact that aforementioned imaging techniques have a higher sensitivity for lesion detection in dense breasts.

\section{Conclusions and future perspectives}

Based on the results from this thesis, the following conclusions as well as suggestions for improving breast cancer detection by digital screening mammography are presented:

1. Radiologist blinded double reading is favoured over radiologist single reading, since this strategy significantly increases cancer detection rate at the expense of an acceptable increase in recall rate and false positive rate. In order to make a definite recommendation, a cost-effectiveness analysis would have to be performed.

2. Blinded double reading generates many discordant recalls. These are mostly based on BI-RADS 0 abnormalities, with a low suspicion for malignancy. Thus a high percentage of discordant recalls represent false positive screens, an undesirable side effect to blinded double reading. The observed difference in mammographic characteristics, namely the higher proportion of asymmetries and architectural distortions among discordant readings, provides possible strategies for improvement. More emphasis could be placed on these 'subtle' mammographic abnormalities when training screening radiologists. Digital breast tomosynthesis has been shown to lower recall rates for asymmetries and decrease inter-observer variability in detecting architectural distortions.

3. Even though previous studies have shown that screening technologists may detect breast cancers that are missed by screening radiologists, their role in arbitration of discordant recalls appears to be limited. Technologist readings of screening mammograms provide no substantial discriminating ability in deciding which discordant readings at radiologist blinded double reading should be recalled. Since this strategy also resulted in a borderline significant decrease in cancer detection rate, we do not advocate it's use in our screening programme.

4. The role of quality assurance sessions in additional cancer detection is limited.

5. Additional ipsilateral and contralateral abnormalities are found through clinical work-up of recalled women. Even though most of these abnormalities prove to be benign, between $15.5 \%$ and $19.0 \%$ represent breast cancers with tumour 
characteristics comparable to screen-detected cancers. A low biopsy threshold for additional abnormalities is warranted in case of an ipsilateral screen-detected cancer. 


\section{References}

1. Bolejko A, Hagell P, Wann-Hansson C, Zackrisson S. Prevalence, long-term development, and predictors of psychosocial consequences of false-positive mammography among women attending population-based screening. Cancer Epidemiol Biomarkers Prev. 2015;24(9):1388-1397.

2. Brodersen J, Siersma VD. Long-term psychosocial consequences of false-positive screening mammography. Ann Fam Med. 2013;11(2):106-115.

3. Van Der Steeg AFW, Keyzer-Dekker CMG, De Vries J, Roukema JA. Effect of abnormal screening mammogram on quality of life. Br J Surg. 2011;98(4):537-542.

4. Posso MC, Puig T, Quintana MJ, Sol??-Roca J, Bonfill X. Double versus single reading of mammograms in a breast cancer screening programme: a cost-consequence analysis. Eur Radiol. 2016;26(9): 3262-3271.

5. Setz-Pels W, Duijm LEM, Coebergh JW, Rutten M, Nederend J, Voogd AC. Re-attendance after falsepositive screening mammography: A population-based study in the Netherlands. $\mathrm{Br} J$ Cancer. 2013;109(8):2044-2050.

6. Perry N, Broeders M, de Wolf C, Törnberg S, Holland R, von Karsa L. European guidelines for quality assurance in breast cancer screening and diagnosis. Fourth edition--summary document. Ann Oncol. 2008;19(4):614-622.

7. Klompenhouwer EG, Voogd AC, Den Heeten GJ, Strobbe LJA, De Haan AFJ, Wauters CA, et al. Blinded double reading yields a higher programme sensitivity than non-blinded double reading at digital screening mammography: A prospected population based study in the south of the Netherlands. Eur J Cancer. 2015;51(3):391-399.

8. Coolen AMP, Voogd AC, Strobbe LJ, Louwman MWJ, Tjan-Heijnen VCG, Duijm LEM. Impact of the second reader on screening outcome at blinded double reading of digital screening mammograms. $\mathrm{Br}$ J Cancer. 2018;119(4):503-507.

9. Posso $M$, Carles $M$, Rué $M$, Puig $T$, Bonfill X. Cost-Effectiveness of Double Reading versus Single Reading of Mammograms in a Breast Cancer Screening Programme. PLoS One. 2016;11(7):e0159806.

10. Klompenhouwer EG, Voogd AC, den Heeten GJ, Strobbe LJA, Tjan-Heijnen VC, Broeders MJM, et al. Discrepant screening mammography assessments at blinded and non-blinded double reading: impact of arbitration by a third reader on screening outcome. Eur Radiol. 2015;25(10):2821-2829.

11. Klompenhouwer EG, Weber RJP, Voogd AC, den Heeten GJ, Strobbe LJA, Broeders MJM, et al. Arbitration of discrepant BI-RADS 0 recalls by a third reader at screening mammography lowers recall rate but not the cancer detection rate and sensitivity at blinded and non-blinded double reading. Breast. 2015;24(5):601-607.

12. Otten JDM, Karssemeijer N, Hendriks JHCL, Groenewoud JH, Fracheboud J, Verbeek ALM, et al. Effect of recall rate on earlier screen detection of breast cancers based on the Dutch performance indicators. Journal of the National Cancer Institute. 2005;97(10):748-754.

13. Welch HG, Prorok PC, O'Malley AJ, Kramer BS. Breast-Cancer Tumor Size, Overdiagnosis, and Mammography Screening Effectiveness. N Engl J Med. 2016;375(15):1438-1447.

14. Lannin DR, Wang S. Are Small Breast Cancers Good because They Are Small or Small because They Are Good? N Engl J Med. 2017;376(23):2286-2291.

15. Coolen AMP, Lameijer JRC, Voogd AC, Louwman MWJ, Strobbe L, Tjan-Heijnen VCG, et al. Characteristics of screen-detected cancers following concordant or discordant recalls at blinded double reading in biennial digital screening mammography. Eur Radiol. 2019;29(1):337-344.

16. van Luijt PA, Heijnsdijk EAM, Fracheboud J, Overbeek LIH, Broeders MJM, Wesseling J, et al. The distribution of ductal carcinoma in situ (DCIS) grade in 4232 women and its impact on overdiagnosis in breast cancer screening. Breast Cancer Res. 2016;18(1):47.

17. Redondo A, Comas M, Macià F, Ferrer F, Murta-Nascimento C, Maristany MT, et al. Inter- and intraradiologist variability in the BI-RADS assessment and breast density categories for screening mammograms. Br J Radiol. 2012;85(1019):1465-1470.

18. Durand MA, Haas BM, Yao X, Geisel JL, Raghu M, Hooley RJ, et al. Early Clinical Experience with Digital Breast Tomosynthesis for Screening Mammography. Radiology. 2015;274(1):85-92. 
19. Dibble EH, Lourenco AP, Baird GL, Ward RC, Maynard AS, Mainiero MB. Comparison of digital mammography and digital breast tomosynthesis in the detection of architectural distortion. Eur Radiol. 2018;28(1):3-10.

20. Ciatto S, Houssami N, Apruzzese A, Bassetti E, Brancato B, Carozzi F, et al. Reader variability in reporting breast imaging according to $\mathrm{BI}-\mathrm{RADS}^{\circledR}$ assessment categories (the Florence experience). Breast. 2006;15(1):44-51.

21. Caumo F, Brunelli S, Tosi E, Teggi S, Bovo C, Bonavina G, et al. On the role of arbitration of discordant double readings of screening mammography: experience from two Italian programmes. Radiol Med. 2011;116(1):84-91.

22. Shaw CM, Flanagan F, Fenlon HM, McNicholas MM. Consensus review of discordant findings maximizes cancer detection rate in double-reader screening mammography: Irish national breast screening program experience. Radiology. 2009;250(2):354-362.

23. Duijm LEM, Groenewoud JH, Fracheboud J, de Koning HJ. Additional double reading of screening mammograms by radiologic technologists: Impact on screening performance parameters. J Natl Cancer Inst. 2007;99(15):1162-1170.

24. Torres-Mejía G, Smith RA, Carranza-Flores M de la L, Bogart A, Martínez-Matsushita L, Miglioretti DL, et al. Radiographers supporting radiologists in the interpretation of screening mammography: a viable strategy to meet the shortage in the number of radiologists. BMC Cancer. 2015;15:410.

25. Coolen AMP, Lameijer JRC, Voogd AC, Strobbe LJ, Louwman MWJ, Tjan-Heijnen VCG, et al. Incorporation of the technologist's opinion for arbitration of discrepant assessments among radiologists at screening mammography. Breast Cancer Res Treat. 2018;171(1):143-149.

26. Pauli R, Hammond S, Cooke J, Ansell J. Comparison of radiographer/radiologist double film reading with single reading in breast cancer screening. J Med Screen. 1996;3(1):18-22.

27. van den Biggelaar FJHM, Nelemans PJ, Flobbe K. Performance of radiographers in mammogram interpretation: A systematic review. Breast. 2008;17(1):85-90.

28. Debono JC, Poulos AE, Houssami N, Turner RM, Boyages J. Evaluation of radiographers' mammography screen-reading accuracy in Australia. J Med Radiat Sci. 2015;62(1):15-22.

29. Tonita JM, Hillis JP, Lim C-H. Medical Radiologic Technologist Review: Effects on a Population-based Breast Cancer Screening Program. Radiology. 1999;211(2):529-533.

30. Coolen AMP, Korte B, Tjan-Heijnen VCG, Bodewes HW, Voogd AC, Duijm LEM. Additional breast cancer detection at digital screening mammography through quality assurance sessions between technologists and radiologists. Radiology. 2020;294(3):509-517.

31. Lameijer JRC, Coolen AM, Voogd AC, Strobbe LJ, Louwman MWJ, Venderink D, et al. Frequency and characteristics of contralateral breast abnormalities following recall at screening mammography. Eur Radiol. 2018;28(10):4205-4214.

32. Lameijer JR, Coolen AMP, Nederend J, Voogd AC, Tjan-Heijnen VC, Duijm LE. Frequency and characteristics of additionally detected ipsilateral breast lesions following recall at screening mammography. Breast. 2018;42:94-101.

33. Setz-Pels W, Duijm LEM, Groenewoud JH, Voogd AC, Jansen FH, Hooijen MJHH, et al. Detection of bilateral breast cancer at biennial screening mammography in the Netherlands: a population-based study. Radiology. 2011;260(2):357-363.

34. Kanumuri P, Hayse B, Killelea BK, Chagpar AB, Horowitz NR, Lannin DR. Characteristics of Multifocal and Multicentric Breast Cancers. Ann Surg Oncol. 2015;22(8):2475-2482.

35. Bernardi D, Gentilini MA, De Nisi M, Pellegrini M, Fantò C, Valentini M, et al. Effect of implementing digital breast tomosynthesis (DBT) instead of mammography on population screening outcomes including interval cancer rates: Results of the Trento DBT pilot evaluation. Breast. 2020;50:135-140.

36. Caumo F, Zorzi M, Brunelli S, Romanucci G, Rella R, Cugola L, et al. Digital breast tomosynthesis with synthesized two-dimensional images versus full-field digital mammography for population screening: Outcomes from the verona screening program. Radiology. 2018;287(1):37-46.

37. Zackrisson S, Lång K, Rosso A, Johnson K, Dustler M, Förnvik D, et al. One-view breast tomosynthesis versus two-view mammography in the Malmö Breast Tomosynthesis Screening Trial (MBTST): a prospective, population-based, diagnostic accuracy study. Lancet Oncol. 2018;19(11):1493-1503. 
38. Lång K, Andersson I, Rosso A, Tingberg A, Timberg P, Zackrisson S. Performance of one-view breast tomosynthesis as a stand-alone breast cancer screening modality: results from the Malmö Breast Tomosynthesis Screening Trial, a population-based study. Eur Radiol. 2016;26(1):184-190.

39. Bernardi $D$, Macaskill $P$, Pellegrini $M$, Valentini $M$, Fantò $C$, Ostillio $L$, et al. Breast cancer screening with tomosynthesis (3D mammography) with acquired or synthetic 2D mammography compared with 2D mammography alone (STORM-2): a population-based prospective study. Lancet Oncol. 2016;17(8):1105-1113.

40. Brennan ME, Houssami N, Lord S, Macaskill P, Irwig L, Dixon JM, et al. Magnetic resonance imaging screening of the contralateral breast in women with newly diagnosed breast cancer: Systematic review and meta-analysis of incremental cancer detection and impact on surgical management. Journal of Clinical Oncology. 2009 Nov 20;27(33):5640-5649.

41. Iacconi C, Galman L, Zheng J, Sacchini V, Sutton EJ, Dershaw D, et al. Multicentric cancer detected at breast mr imaging and not at mammography: Important or not? Radiology. 2016;279(2):378-384.

42. Melnikow J, Fenton JJ, Whitlock EP, Miglioretti DL, Weyrich MS, Thompson JH, et al. Supplemental screening for breast cancer in women with dense breasts: A systematic review for the U.S. Preventive services task force. Annals of Internal Medicine. 2016;164(4):268-278.

43. Mann RM, Balleyguier C, Baltzer PA, Bick U, Colin C, Cornford E, et al. Breast MRI: EUSOBI recommendations for women's information. Eur Radiol. 2015;25(12):3669-3678.

44. Nortier JWR, Rutgers EJT, Sangen MJC van der, Tienhoven G van, Vegchel T van, Zonderland HM, et al. Richtlijn Mammacarcinoom versie 2.0. Oncoline. 2012;

45. Bakker MF, de Lange S V., Pijnappel RM, Mann RM, Peeters PHM, Monninkhof EM, et al. Supplemental MRI Screening for Women with Extremely Dense Breast Tissue. N Engl J Med. 2019;381(22):2091-2102. 
Summary 



\section{Summary}

\section{Radiologist blinded double reading}

The first objective of this thesis was to determine the impact of the second reader on screening outcome (chapter 2 ). We found that adding a second reader resulted in a significant increase in cancer detection rate (from 6.2 per 1000 screens to 7.0 per 1000 screens, $p<0.001$ ) at the expense of an acceptable increase in recall rate (from $3.0 \%$ to $3.6 \%, p<0.001$ ) and false positive recall rate (from 23.4 per 1000 screens to 28.7 per 1000 screens, $p<0.001$ ). Based on these results we therefore favour blinded double reading over single reading.

In addition to the overall screening outcome, we also compared mammographic and tumour characteristics between first and second reader recalls. We found a larger proportion of BI-RADS 0 lesions in de group of women additionally recalled by the second reader and recalled lesions comprised larger proportions of asymmetries and architectural distortions. The majority of 82 cancers additionally detected by the second reader were small ( $86.9 \% \mathrm{~T} 1 \mathrm{a}-\mathrm{c})$ invasive cancers, mostly of low histological grade $(59.0 \%$ grade $\mathrm{I})$.

Because of our study design, additional recalls by the second reader are by definition discordant recalls. However, the second reader might not agree with all recall decisions made by the first reader (i.e. there are also discordant readings among first reader recalls). In chapter 3 we further analysed this and determined which mammographic and tumour characteristics led to concordant versus discordant readings. A discordant reading was defined as a difference in classification by two radiologists where one classified it as BI-RADS 1 or 2 (negative, i.e. no recall) and the other as BI-RADS 0, 4 or 5 (positive, i.e., recall). In case of a discordant reading, the woman in question was always recalled for further analysis. The majority of recalls (71.8\%) were based on a concordant reading. The PPV of a concordant recall was higher compared with a discordant recall $(23.5 \%$ vs. $10.0 \%, p<0.001)$. As we expected based on the results presented in chapter 2, the proportion of BI-RADS 0 recalls was significantly higher in the discordant reading group $(75.7 \%$ vs. $56.3 \%, p<0.001)$ and discordant readings were more often an asymmetry or architectural distortion $(21.8 \%$ vs. $13.2 \%$ and $9.3 \%$ vs. $6.8 \%$, respectively; $p<0.001)$. Invasive cancers detected through discordant recalls showed a more favourable (grade I) tumour grade $(54.7 \%$ vs. $39.9 \%, p=0.022$ ). There was also a trend towards smaller tumours with fewer cancers showing lymph node metastases $(16.0 \%$ vs. $23.5 \%, p=0.117)$. Other tumour characteristics were comparable for both groups. 


\section{Value of technologist's reading and quality assurance sessions}

Since the initiation of our screening programme, screening technologists have been encouraged to look for mammographic abnormalities. Their training not only focuses on mammography technique and positioning but also on breast anatomy and pathology (benign and malignant). After obtaining a mammogram, they review it and annotate any abnormality for which they consider recall necessary.

In chapter 4.1 we determined whether this technologist reading could be used to decide if discordant readings at radiologist blinded double reading should be recalled. We found that the proportion of breast cancer cases among discordant recalls was comparable for women with or without a positive technologist reading, irrespective of BI-RADS classification (5.0\% vs. $4.6 \%$ for BI-RADS 0 recalls, $p=0.8$; and $28.9 \%$ vs. $25.4 \%$ for BI-RADS 4 or 5 recalls, $p=0.5$ ). This indicates that, in deciding whether a discordant reading should be recalled, assessment by a technologist does not provide a significant discriminating ability. When also considering the, borderline significant, decrease in cancer detection rate, there appears to be no added value of using the technologist's assessment of screening mammograms in arbitrating discordant readings.

For the purpose of quality assurance and continued training, technologists attend 6-weekly sessions where a coordinating screening radiologist discusses all mammograms that were considered suspicious by the technologists but were not recalled by the screening radiologists. Additional tumours are detected through these quality assurance sessions and in chapter 4.2 we determined the frequency and characteristics of these cancers. Over 466,000 mammograms were included, resulting in 14,142 recalls and 3156 screen-detected cancers. During our 8-year inclusion period, technologists would have recalled 11,627 women with a negative radiologist screen (i.e., BI-RADS 1 or 2). All these mammograms were discussed at quality assurance sessions, resulting in 85 recalls and 26 screen-detected cancers $(0.8 \%$ of all screen-detected cancers). Most of these cancers were invasive and larger than $10 \mathrm{~mm}$ in size, therefore probably not reflecting overdiagnosis. Sensitivity of quality assurance sessions for additional cancer detection was 52\% (26 of 50; 95\% confidence interval: $38 \%, 66 \%)$. We found no significant differences in mammographic and tumour characteristics compared with the 3130 screen-detected cancers detected through standard radiologist double reading. We also compared the cancers detected through quality assurance sessions with interval cancers and screen-detected cancers with a technologist positive screen for the same abnormality in a previous screening round (i.e., 'missed' at quality assurance sessions) and also found no significant differences. The role of these sessions in additional cancer detection appears to be 
limited. However, they serve many other purposes, such as continued training, discussing interesting and instructive cases and receiving feedback on performance.

\section{Frequency and characteristics of additional breast abnormalities following screening mammography}

In clinical work-up after recall, additional breast cancers may be detected in the contralateral and/or ipsilateral breast. We studied the frequency and outcome of additionally detected contralateral (chapter 5.1) and ipsilateral (chapter 5.2) breast abnormalities following recall. We found that 3.2\% of recalled women underwent assessment of a contralateral, non-recalled breast abnormality and $6.4 \%$ of recalled women was assessed for a ipsilateral, non-recalled lesion. In both groups, the additional lesion was in most cases first detected by review of the screening mammogram or additional mammographic views, with or without breast tomosynthesis. Since tomosynthesis is increasingly being used in clinical and, to a lesser extent, screening setting, it is expected that the number of additional lesions detected after recall will increase. Women with an additionally detected breast abnormality more often had high breast density (ACR III + IV), 38.8\% vs. $23.8 \%$ for contralateral lesions $(p<0.001)$ and $29.0 \%$ vs. $23.8 \%$ for ipsilateral lesions $(p=0.06)$. The majority of additionally detected lesions were benign and mostly comprised cysts and fibroadenomas. Additionally detected lesions proved to be malignant in $19.0 \%$ (ipsilateral) and $15.5 \%$ (contralateral) of cases, respectively. Most additional ipsilateral malignancies were part of a multifocal or multicentric screen-detected tumour. Positive predictive value of biopsy was higher for recalled lesions compared with nonrecalled lesions. However, in case of an additional ipsilateral lesion, PPV of biopsy was higher in women with a malignant recalled lesion compared to benign recalled lesions. The latter implicates that a low biopsy threshold is warranted in case of a malignant index lesion. 

Impact paragraph 



\section{Impact paragraph}

Breast cancer is the most common cancer diagnosed in women worldwide, affecting one in seven Dutch women during her lifetime. The Dutch nationwide breast cancer screening programme was gradually implemented between 1989 and 1996, aiming to reduce breast cancer mortality through early detection and thereby early treatment. Indeed, the combination of early detection through screening and substantial improvements in treatment, has resulted in a substantial decline in breast cancer mortality in the last three decades. In 2014, the Dutch Health Council published a report concluding that the screening programme is as effective as was originally expected and that the benefits relevantly outweigh the harms. These harms are mainly overdiagnosis (i.e., a woman is diagnosed with a breast cancer that would not have become clinically relevant during her lifetime) and false positive screening results (i.e., a woman is referred for further evaluation of an abnormality that does not turn out to be breast cancer). A delicate balance exists between different performance indicators of breast cancer screening, namely recall rate (percentage of screened women referred with a positive screening examination), cancer detection rate and false positive recall rate. In order for the screening programme to remain effective for years to come, continued evaluation and optimisation of these indicators is of utmost importance.

In this thesis, we therefore evaluated several aspects of the Dutch screening programme, aiming to provide opportunities for further improvement. Studies in the first part of this thesis focus on mammography reading strategies. Double reading by two screening radiologists is considered standard of care in Dutch and European guidelines. Previous studies comparing single reading to double reading mostly employ non-blinded double reading and date from the era of screen-film mammography as opposed to digital mammography which is currently the standard. Based on the results from these studies, showing an increased cancer detection with double reading, one would expect a comparable or even greater benefit with blinded double reading of digital screening mammograms. However, studies addressing this subject are lacking and it was therefore subject of study in this thesis. Our results confirm a significant increase in cancer detection with blinded double reading, at the expense of an acceptable increase in the number of recalls and false positive recalls. We also found that in most cases where two radiologists disagree on whether or not a woman should be recalled (a discordant recall), this concerns an abnormality with a low suspicion for malignancy. These discordant recalls are therefore more likely to be false positive recalls. False positive recalls cause psychological distress in women involved and add to health care costs. In order to decrease false positive recall rates and thereby improve breast cancer screening, it is important to gain insight in what causes a discordant recall. We compared concordant and discordant recalls and found 
that there are no differences in the characteristics of breast cancers that are detected, except for a more favourable tumour grade in case of a discordant recall. We did find a difference in how these tumours appear on the screening mammogram. The percentage of 'subtle' mammographic abnormalities was higher in the group of discordant recalls. Additional emphasis on the detection and classification of these subtle abnormalities (architectural distortions and asymmetries) in training screening radiologists might therefore be beneficial. There might also be a role for digital breast tomosynthesis, which has been proven to better depict these abnormalities than standard digital mammography.

The second part of this thesis focussed on the role of screening technologists' reading and training on screening outcome. One of our aims was to study whether the technologist's reading of screening mammograms could be used to decide which discordant radiologist readings should be recalled, thereby hopefully decreasing recall rates and false positive recalls. Unfortunately, our findings indicate that the technologist's reading provides no substantial discriminating ability in case of a discordant reading between two radiologists. Although aforementioned strategy would lower recall rates and false positive recall rates, this lack of discriminating ability combined with a decrease in cancer detection rate leads to our conclusion that it has no added value in the Dutch screening programme. We also studied the role of 6-weekly quality assurance sessions (which, among other things, serve as training sessions for screening technologists) in additional cancer detection and found that it has limited value.

In the third part of this thesis, we focussed on additional breast abnormalities that are found during the clinical work-up of recalled women after recall. We found that even though most of these abnormalities prove to be benign, they do comprise breast cancers in about 15 to $20 \%$ of cases and tumour characteristics do not differ significantly from those of breast cancers detected through screening. In women with a breast cancer detected through screening and an additional abnormality in the same breast, positive predictive value of biopsy of this additional abnormality was higher compared to women with a benign recalled lesion and an additional abnormality.

Results from this thesis are relevant to health care professionals involved in breast cancer screening and breast cancer care. As mentioned before, it is important to continuously evaluate and optimise the screening programme, in order for it to remain effective. The currently employed reading strategy, radiologist blinded double reading, is indeed superior to single reading and we aimed to provide insight into causes of discordant recalls between radiologists, providing possible strategies to decrease false positive recalls. The latter is also very relevant to women participating in the screening programme because they are likely to experience psychological 
distress following a false positive recall. For both screening radiologists and clinical radiologists involved in the workup of recalled women, we stress the importance of careful evaluation of both breasts on mammography, especially in the presence of an abnormality. This vigilance would limit so called 'satisfaction of search', where radiologists, when having found one abnormality overlook a second one. We also advise clinical radiologists to maintain a low threshold for biopsy of an additionally detected abnormality in women with a screen-detected breast cancer.

Apart from being published in international peer-reviewed journals, parts of the research presented in this thesis have been presented at national and international meetings. Results from chapter 4.2 have been published in the newsletter from the Netherlands Comprehensive Cancer Organisation (IKNL) and were also featured on a large community website for medical imaging professionals worldwide. Results from this thesis have thereby been made available to health care professionals both nationally and internationally. 

Dankwoord 



\section{Dankwoord}

De combinatie van een promotietraject met een drukke baan en een jong gezin bleek op z'n zachtst gezegd een uitdaging. Een uitdaging die ik zonder alle hulp van de mensen om me heen nooit had kunnen volbrengen. Graag zou ik enkele van hen in het bijzonder bedanken.

Allereerst natuurlijk mijn promotor en copromotoren:

Prof. dr. V.C.G. Tjan-Heijnen, beste Vivianne. 'Het mag kort en bondig', het klonk me als muziek in de oren! Met je snelle, waardevolle commentaar, frisse kijk en pragmatische aanpak heb je me enorm geholpen, dankje!

Dr. L.E.M. Duijm, beste Lucien. Ik heb me keer op keer verbaasd over je tomeloze energie en inzet en me af en toe een tikkeltje moedeloos gevoeld als je binnen een uur al een volledig gecorrigeerd stuk retour mailde. Maar, bovenal ben ik je steeds dankbaar geweest dat je ervoor zorgde dat deze trein, no matter what, altijd bleef rijden. Je bent de beste begeleider geweest die ik me had kunnen wensen!

Dr. A.C. Voogd, beste Adri, bedankt voor alle correcties en goede adviezen. Je oprechte interesse en de bemoedigende woorden als het me even te veel werd, hebben me geholpen dit alles tot een goed einde te brengen.

De leden van de beoordelingscommissie, prof. dr. Smidt, prof. dr. Pijnappel, prof. dr. Broeders, prof. dr. Muris en prof. dr. Boersma, ik wil jullie hartelijk bedanken dat jullie de tijd hebben genomen om mijn proefschrift inhoudelijk te beoordelen.

Dank aan alle screeningsradiologen, laboranten, data analisten en administratief medewerkers van onze screeningsregio. Jullie werk ligt aan de basis van dit proefschrift.

Dank aan alle mede auteurs, Joost Lameijer, Luc Strobbe, Marieke Louwman, Bram Korte, Hans Bodewes, Dick Venderink en Joost Nederend.

Dr. P.N.M. Lohle, beste Paul: de aanhouder wint! Al in het eerste jaar van mijn opleiding tot radioloog probeerde je me over te halen om te promoveren, in het vierde jaar ben ik pas gezwicht. Ik ben je dankbaar voor het vertrouwen dat je al deze tijd in me hebt gehad en voor deze welgemeende 'schop onder de kont', waardoor ik verder ben gekomen dan ik ooit had gedacht. 
Dank aan alle andere opleiders, inmiddels collega radiologen, van de vakgroep radiologie in het ETZ. Mede dankzij jullie heb ik een fantastische, leerzame en gezellige opleidingstijd gehad. Ik heb enorm veel zin om met jullie, en alle nieuwe collega's, verder te bouwen op de basis die jullie hebben gelegd.

Lieve Irene, ik heb je de laatste jaren leren kennen als een ambitieus, vriendelijk en ontzettend attent persoon. Jouw goede adviezen, bemoedigende woorden en gezelligheid bij een kop thee hebben hebben me (zeker het afgelopen jaar) meer geholpen dan je je waarschijnlijk beseft. Je bent een hele fijne collega, en een nog fijnere vriendin!

Lieve Claudia, het is al bijna 8 jaar geleden dat we elkaar ontmoetten. Wat een opleidingstijd hebben we samen gehad! Als collega's, én als vriendinnen. Van rosé op het strand tot samen door een typische EMC dienst 'vliegen', en van kopjes thee tijdens ons verlof tot die achterste rij stoelen bij het blokonderwijs. De opleiding zit er inmiddels op, maar er liggen gelukkig nog genoeg glazen thee en rosé in het verschiet! Met jullie als paranimfen wordt deze dag zeker een groot succes!

Lieve Harry, vanaf het eerste moment heb ik me welkom gevoeld in jullie gezin, iets waar ik jou en Ineke nog steeds heel dankbaar voor ben. Je geniet zichtbaar van alle tijd met je kleindochters, en wij genieten op onze beurt van de wekelijkse gezelligheid met jou!

Lieve oma, in gedachte bent u erbij, hier in uw 'Sjoen Mestreech', uiteraard op de eerste rij en met een trotse glimlach.

Lieve Martijn, ik kijk met heel veel trots naar hoe goed het met je gaat en geniet ervan om je te zien als lieve, trotse oom.

Lieve papa, je bent altijd vriendelijk en zorgzaam voor de mensen om je heen en dankzij jouw harde werken heeft het me nooit aan iets ontbroken. Mede daardoor ben ik gekomen waar ik nu ben!

Lieve mama, ons huis was dankzij jou een warm thuis, altijd gevuld met liefde, muziek, boeken en gezelligheid. Ik mis je, vaak op de gekste momenten, en vandaag een klein beetje extra.

Lieve Abby en Evie, wat een gezelligheid en energie brengen jullie in huis! Abby, hopelijk blijf je altijd dansen door het leven. Evie, ook al ben je nog zo klein, je lach straalt nu al een en al ondeugd uit, dat belooft wat! 
Lieve Bas, ik weet niet of je helemaal wist waar we aan begonnen toen je enthousiast zei: 'Een promotietraject? Ja hoor, doen!'. Nu, bijna 5 jaar later, ben je hopelijk net zo trots als ik ben op het eindresultaat! Je hebt me geholpen om alle balletjes hoog te houden én, niet te vergeten, om die enorme database de baas te worden. Het is naïef om te denken dat nu alles rustiger wordt, maar één ding weet ik zeker: wát er ook op ons pad komt, met jou is alles leuker!!! 

Curriculum vitae 



\section{Curriculum vitae}

Angela Maria Petrus Coolen werd op 7 april 1988 geboren in Eindhoven. In 2007 behaalde zij haar VWO diploma aan het Augustinianum in Eindhoven waarna zij in september 2007 startte met de opleiding geneeskunde aan de Universiteit Maastricht. Tijdens een keuze coschap radiologie in het toenmalige St. Elisabeth ziekenhuis in Tilburg werd de interesse in de radiologie, in het bijzonder de neuroradiologie, definitief gewekt. In het laatste jaar van haar geneeskundeopleiding heeft ze daarom een gecombineerde klinische en wetenschappelijke stage radiologie gevolgd in het MUMC te Maastricht. Enkele maanden na het behalen van haar artsexamen begon zij op 1 oktober 2013 met de opleiding tot radioloog in het St. Elisabeth ziekenhuis (later Elisabeth-Tweesteden ziekenhuis, ETZ) in Tilburg en het Erasmus MC in Rotterdam, welke zij in februari 2019 afrondde. Tijdens deze opleiding heeft zij zich verder verdiept in de mammaradiologie met als tweede aandachtsgebied de neuro- en hoofd/hals radiologie. In het vierde jaar van de opleiding begon zij onder begeleiding van dr. L.E.M. Duijm aan het onderzoek dat resulteerde in dit proefschrift (promotor prof. dr. V.C.G. Tjan-Heijnen, co-promoteren dr. L.E.M. Duijm en dr. A.C. Voogd). Momenteel is ze werkzaam als radioloog binnen de vakgroep radiologie in het ETZ en bevindt zij zich in de afrondende fase van het fellowship neuro en hoofd/hals radiologie. 


